\title{
MEASURES OF WEAK NON-COMPACTNESS IN PREDUALS OF VON NEUMANN ALGEBRAS AND JBW*-TRIPLES
}

\author{
JAN HAMHALTER, ONDŘEJ F.K. KALENDA, ANTONIO M. PERALTA, \\ AND HERMANN PFITZNER
}

\begin{abstract}
We prove, among other results, that three standard measures of weak non-compactness coincide in preduals of $\mathrm{JBW}^{*}$-triples. This result is new even for preduals of von Neumann algebras. We further provide a characterization of $\mathrm{JBW}^{*}$-triples with strongly WCG predual and describe the order of seminorms defining the strong* topology. As a byproduct we improve a characterization of weakly compact subsets of a $\mathrm{JBW}^{*}$-triple predual, providing so a proof for a conjecture, open for almost eighteen years, on weakly compact operators from a $\mathrm{JB}^{*}$-triple into a complex Banach space.
\end{abstract}

\section{INTRODUCTION}

Measures of weak non-compactness are an important tool for a deeper understanding of weak compactness of sets and operators. They are used to prove more precise versions of known results and to establish new results as well. As an illustration we mention a fixed-point theorem [21, quantitative versions of Krein's theorem [26, 31, James' compactness theorem [18, 32, Eberlein-Šmulyan theorem [3] and Gantmacher's theorem [4.

There are several procedures how one can measure weak non-compactness of sets. There are two basic non-equivalent ways - on the one hand the De Blasi measure introduced and used in 21, and on the other hand various mutually equivalent quantities used in the other above-quoted papers. Their non-equivalence follows from 5, 4. However, the counterexample witnessing their non-equivalence is an artificially constructed Banach space - it is constructed as the $\ell^{1}$-sum of suitable renormings of the space $\ell^{1}$. It seems to be still an open question whether there is a 'natural' Banach space where they fail to be equivalent.

This question seems to be rather interesting as in many classical spaces all these measures of weak non-compactness are equivalent. This applies, for example, to the Lebesgue spaces $L^{1}(\mu)$ for an arbitrary non-negative $\sigma$-additive measure $\mu$ [6. Theorem 7.5], including the special case $\ell^{1}(\Gamma)$ [46, Proposition 7.3], the space $c_{0}(\Gamma)$ [46, Proposition 10.2], the space of nuclear operators $N\left(\ell^{q}(\Lambda), \ell^{p}(J)\right)$ for $p, q \in(1, \infty)$

2010 Mathematics Subject Classification. 46B50, 46L70, 17C65.

Key words and phrases. measure of weak non-compactness, von Neumann algebra, JBW*triple, $\mathrm{JBW}^{*}$-algebra, strong* topology.

The first two authors were in part supported by the Research Grant GAČR 17-00941S. The first author was partly supported further by the project OP VVV Center for Advanced Applied Science CZ.02.1.01/0.0/0.0/16_019/000077. The third author was partially supported by the Spanish Ministry of Science, Innovation and Universities (MICINN) and European Regional Development Fund project no. PGC2018-093332-B-I00 and Junta de Andalucía grant FQM375. 
34. Theorem 2.1], and preduals of atomic von Neumann algebras 34, Theorem $2.2]$.

In the present paper we prove the equivalence of the measures of weak noncompactness in preduals of $\mathrm{JBW}^{*}$-triples. This covers, in particular, the preduals of general von Neumann algebras.

The machinery developed in this paper also have some important consequences in improving our current knowledge on relatively weakly compact subsets in the predual of a $\mathrm{JBW}^{*}$-triple. More concretely, the result established in 62 asserts that a bounded subset, $K$, in the predual of a $\mathrm{JBW}^{*}$-triple, $M$, is relatively weakly compact if and only if there is a couple of normal functionals $\varphi_{1}, \varphi_{2}$ in $M_{*}$ whose associated preHilbertian seminorm $\|\cdot\|_{\varphi_{1}, \varphi_{2}}$ controls uniformly the values of all functionals in $K$ on the closed unit ball of $M$ (see sections 3, 6, and 7 for definitions). As shown, for example in [17, pages 340 and 341], the existence of control seminorms $\|\cdot\|_{\varphi_{1}, \varphi_{2}}$ for relatively weakly compact subsets in $M_{*}$ can be applied to characterize weakly compact operators from a complex Banach space into the predual of a $\mathrm{JBW}^{*}$-triple and from a $\mathrm{JB}^{*}$-triple into a complex Banach space. It has been conjectured that, as in the case of von Neumann algebras (see [1, 45]), a single functional $\varphi$ in the predual, $M_{*}$, of a $\mathrm{JBW}^{*}$-triple $M$ (and the associated seminorm $\|\cdot\|_{\varphi}$ ) is enough to control relatively weakly compact subsets in $M_{*}$ and to determine those weakly compact operators from a complex Banach space into $M_{*}$. It was even so claimed in [20, Theorem 11]. However, some subtle difficulties in the BartonFriedman's proof for the Grothendieck inequality for JB*-triples, also affected the arguments in 20] (cf. section 11] or [17, page 341]). In this paper we also provide a complete proof for this conjecture and we show the validity of the original statement in [20, Theorem 11].

The paper is organized as follows.

In Section 2 we give the definitions of the three measures of weak non-compactness we will deal with and provide their basic properties.

Section 3 contains some background on $\mathrm{JB}^{*}$-triples and, more specifically, on dual spaces among them, i.e., JBW*-triples. We provide the basic notions and properties, including the relationship to the classical subclasses formed by $\mathrm{C}^{*}$-algebras (respectively, von Neumann algebras) and $\mathrm{JB}^{*}$-algebras (respectively, $\mathrm{JBW}^{*}$-algebras).

In Section 4 we formulate our main result on the equivalence of the three measure of weak non-compactness in $\mathrm{JBW}^{*}$-triple preduals (see Theorem 4.1). We further collect some consequences - the special cases of the main result and the analogous result for real spaces.

Sections 5, 6, 7 and 8 are devoted to the proof of the main result. On the way to the proof we establish several results which seem to be of an independent interest. In Section 5 we show that there is a close relationship between the equality of measures of weak non-compactness and the subsequence splitting property (see Proposition 5.1).

In Section 6 we gather some properties of projections in $\mathrm{C}^{*}$-algebras and $\mathrm{JB}^{*}$ algebras and of tripotents in $\mathrm{JB}^{*}$-triples. Most of the results presented there are known, but we point out that Lemmata 6.1 and 6.3 and Proposition 6.5 seem to be of independent interest.

In Section 7 we investigate the relation between the strong* topology on a JBW*triple and the weakly compact subsets of its predual. The main achievements there 
are Propositions 7.5 and 7.11, where we explore the connections between the natural partial order in the set of tripotents with the order among the associated seminorms and the first results leading to the existence of control functionals.

Section 8 contains the culminating arguments of the proof of Theorem 4.1

In Section 9 we focus on $\sigma$-finite $\mathrm{JBW}^{*}$-triples. We characterize those $\sigma$-finite $\mathrm{JBW}^{*}$-triples whose predual is strongly WCG (see Theorem 9.3). This theorem reveals, in particular, a substantial difference between $\mathrm{JBW}^{*}$-triples and $\mathrm{JBW}^{*}$ algebras, as the predual of a $\sigma$-finite $\mathrm{JBW}^{*}$-algebra is always strongly WCG.

In Section 10 we apply the methods from Section 9 to prove Theorem 10.1 which provides a deep understanding of the structure of the strong* topology for general $\mathrm{JBW}^{*}$-triples.

In Section 11 we present the new advances on the characterization of relatively weakly compact subsets in the predual of a $\mathrm{JBW}^{*}$-triple (see Theorem 11.3), and the subsequent consequences determining the weakly compact operators from a JB*-triple into a complex Banach space (cf. Theorem 11.4), which, as we have already commented, provides a proof for a conjecture considered during the last eighteen years.

The last section contains some open problems.

\section{MeAsures of WEAK NONCOMPACTNESS}

Let $X$ be a (real or complex) Banach space and $A, B \subseteq X$ two nonempty sets. We set

$$
\widehat{\mathrm{d}}(A, B)=\sup \{\operatorname{dist}(a, B) ; a \in A\} .
$$

The Hausdorff measure of norm non-compactness is defined by the formula

$$
\chi(A)=\inf \{\widehat{\mathrm{d}}(A, F) ; F \subseteq X \text { finite }\}=\inf \{\widehat{\mathrm{d}}(A, K) ; K \subseteq X \text { compact }\}
$$

for a bounded set $A \subseteq X$. It is clear that $\chi(A)=0$ if and only if $A$ is relatively norm compact.

The De Blasi measure of weak non-compactness introduced in [21] is defined by

$$
\omega(A)=\inf \{\widehat{\mathrm{d}}(A, K) ; K \subseteq X \text { weakly compact }\} .
$$

(When confusion concerning the underlying space $X$ could arise, like for example in Lemma 2.1. we will write $\omega_{X}$ instead of $\omega$. ) It is a natural modification of the Hausdorff measure of non-compactness. Further, $\omega(A)=0$ if and only if $A$ is relatively weakly compact. As remarked in 21 this was proved already by Grothendieck [33, p. 401] (compare also [22, Lemma XIII.2]).

Another measure of weak non-compactness inspired by the Banach-Alaoglu theorem is defined by

$$
\mathrm{wk}_{X}(A)=\widehat{\mathrm{d}}\left(\bar{A}^{w^{*}}, X\right) .
$$

Here $\bar{A}^{w^{*}}$ is the closure of $A$ in the space $\left(X^{* *}, w^{*}\right)$, where $X$ is considered to be canonically embedded into its bidual. It is clear that $A$ is relatively weakly compact if and only if $\bar{A}^{w^{*}} \subseteq X$, i.e., if and only if $\mathrm{wk}_{X}(A)=0$.

We will use one more quantity, namely

$$
\operatorname{wck}_{X}(A)=\sup \left\{\operatorname{dist}\left(\operatorname{clust}_{w^{*}}\left(x_{n}\right), X\right) ;\left(x_{n}\right) \text { is a sequence in } A\right\},
$$

where $\operatorname{clust}_{w^{*}}\left(x_{n}\right)$ denotes the set of all weak*-cluster points of the sequence $\left(x_{n}\right)$ in the bidual $X^{* *}$. It follows easily from the Eberlein-ŠSmulyan theorem that 
wck $_{X}(A)=0$ whenever $A$ is relatively weakly compact. The converse follows from the quantitative version of the Eberlein-Šmulyan theorem proven in [3]. It consists in the inequalities

$$
\operatorname{wck}_{X}(A) \leq \operatorname{wk}_{X}(A) \leq 2 \operatorname{wck}_{X}(A)
$$

which hold for any bounded subset $A \subseteq X$. Further, the following inequalities are easy to check:

$$
\operatorname{wk}_{X}(A) \leq \omega(A) \leq \chi(A) .
$$

In general, the quantities $\operatorname{wk}_{X}(\cdot)$ and $\omega(\cdot)$ (hence also $\operatorname{wck}_{X}(\cdot)$ and $\omega(\cdot)$ ) are not equivalent. As mentioned above, this was proved in [5, 4] but it follows also from [46. Theorem 2.3] using the fact that weakly compactly generated spaces are not stable under taking subspaces.

If $Y$ is a closed subspace of $X$ and $A$ is a bounded subset of $Y$, then we can consider the measures of weak non-compactness of the set $A$ either in the space $X$ or in the space $Y$. In general it is not the same but we have

$$
\operatorname{wk}_{X}(A) \leq \operatorname{wk}_{Y}(A) \leq 2 \operatorname{wk}_{X}(A), \quad \operatorname{wck}_{X}(A) \leq \operatorname{wck}_{Y}(A) \leq 2 \operatorname{wck}_{X}(A) .
$$

Indeed, in both cases the first inequality is trivial and the second one follows from [31, Lemma 11].

Further, we clearly have $\omega_{X}(A) \leq \omega_{Y}(A)$ but the converse inequality is, in general, not true, neither up to a constant (this follows, for example, from [46, Theorem 2.3] with the same remark as above).

However, if $Y$ is 1-complemented in $X$, then the measures considered in $X$ and in $Y$ coincide. This is the content of the following easy lemma which is proved in [34, Lemma 3.8(b)].

Lemma 2.1. Let $X$ be a Banach space, $Y \subseteq X$ a 1-complemented subspace and $A \subseteq Y$ a bounded set. Then

$$
\operatorname{wk}_{X}(A)=\operatorname{wk}_{Y}(A), \quad \operatorname{wck}_{X}(A)=\operatorname{wck}_{Y}(A), \quad \omega_{X}(A)=\omega_{Y}(A) .
$$

\section{JB*-TRIPLES AND THEIR SUBCLASSES}

Originated as mathematical models of physical observables in quantum mechanics by authors like W. Heisenberg, P. Jordan and J. von Neumann, C*-algebras constitute nowadays an area of intensive research in functional analysis. The abstract definition says that a $C^{*}$-algebra is a complex Banach algebra $A$ equipped with an algebra involution * satisfying the so-called Gelfand-Naimark axiom, that is $\left\|a^{*} a\right\|=\|a\|^{2}$ for all $a \in A$. The celebrated Gelfand-Naimark theorem represents each abstract $\mathrm{C}^{*}$-algebra as a norm-closed selfadjoint subalgebra of the space $B(H)$ of all continuous linear operators on a complex Hilbert space $H$.

From the point of view of functional analysis, the class of $\mathrm{C}^{*}$-algebras is not very stable for several properties. For example, a $\mathrm{C}^{*}$-algebra is reflexive if and only if it is finite-dimensional, and $\mathrm{C}^{*}$-algebras are not stable under contractive projections as we can find a contractive projection from $B(H)$ onto the Hilbert space $H$. As observed by Poincaré in 1907, for complex Banach spaces of dimension bigger than or equal to two, there exists a wide range of simply connected domains which are not biholomorphic to the unit ball. In other words, the Riemann mapping theorem cannot be easily generalized to arbitrary complex Banach spaces. JB*triples were introduced in 1983 by W. Kaup in a successful classification of bounded 
symmetric domains in complex Banach spaces of arbitrary dimension (see [52]). A $J B^{*}$-triple is a complex Banach space $E$ together with a (continuous) triple product $\{., .,\}:. E^{3} \rightarrow E$, which is symmetric and bilinear in the outer variables and conjugate-linear in the middle one, satisfying the following algebraic-analytic properties:

$\left(J B^{*}-1\right) \quad L(x, y) L(a, b)=L(L(x, y)(a), b)-L(a, L(y, x)(b))+L(a, b) L(x, y)$ for all $a, b, x, y \in E$, where given $a, b \in E, L(a, b)$ stands for the (linear) operator on $E$ given by $L(a, b)(x)=\{a, b, x\}$, for all $x \in E$ (Jordan identity);

$\left(J B^{*}-2\right)$ The operator $L(a, a)$ is a hermitian operator with nonnegative spectrum for each $a \in E$;

$\left(J B^{*}-3\right)\|\{a, a, a\}\|=\|a\|^{3}$ for $a \in E$.

Let us observe that the third axiom $\left(J B^{*}-3\right)$ is a Jordan-geometric analogue of the Gelfand-Naimark axiom.

The mapping $(x, y, z) \mapsto\{x, y, z\}=\frac{1}{2}\left(x y^{*} z+z y^{*} x\right)$ can be applied to define a structure of $\mathrm{JB}^{*}$-triple on a $\mathrm{C}^{*}$-algebra $A$, or on the space $B(H, K)$ of all bounded linear operators between complex Hilbert spaces $H$ and $K$ and in particular on an arbitrary Hilbert space $H$ identified with $B(H, \mathbb{C})$, in all these cases we keep the original norm of the corresponding underlying Banach spaces.

Some of the lackings exhibited by the class of $\mathrm{C}^{*}$-algebras are no longer a handicap for $\mathrm{JB}^{*}$-triples. For example, $\mathrm{JB}^{*}$-triples are stable under contractive perturbations (see [28, 75, [53]). One of the most interesting properties of $\mathrm{JB}^{*}$-triples is that a linear bijection between $\mathrm{JB}^{*}$-triples is an isometry if and only if it is a triple isomorphism, that is, it preserves triple products (see [52, Proposition 5.5]). In particular, if a complex Banach space $E$ admits two triple products under which $E$ is a $\mathrm{JB}^{*}$-triple with respect to its original norm and any of these products, then both products coincide.

An intermediate class between $\mathrm{C}^{*}$-algebras and $\mathrm{JB}^{*}$-triples is formed by $\mathrm{JB}^{*}$ algebras. The hermitian part, $A_{s a}$, of a $\mathrm{C}^{*}$-algebra $A$ is not, in general, closed under products. However $A_{s a}$ is a Jordan subalgebra of $A$ when the latter is considered with its natural Jordan product defined by $a \circ b=\frac{1}{2}(a b+b a)$.

P. Jordan released the notion of Jordan algebra to set a mathematical model for the algebra of observables in quantum mechanics. In abstract algebra, a Jordan algebra is a nonassociative algebra over a field whose multiplication, denoted by $\circ$, is commutative and satisfies the so-called Jordan identity $(x \circ y) \circ x^{2}=x \circ\left(y \circ x^{2}\right)$. Given an element $a$ in a Jordan algebra $B$, we shall write $U_{a}$ for the linear mapping on $B$ defined by $U_{a}(b):=2(a \circ b) \circ a-a^{2} \circ b$. A Jordan Banach algebra $B$ is a Jordan algebra equipped with a complete norm satisfying $\|a \circ b\| \leq\|a\| \cdot\|b\|$ for all $a, b \in B$. A complex Jordan Banach algebra $B$ admitting an involution * satisfying that $\left\|U_{a}\left(a^{*}\right)\right\|=\|a\|^{3}$ for all $a, b \in B$ is called a $J B^{*}$-algebra (see [79, [16. Definition 3.3.1]). In some texts the definition of $\mathrm{JB}^{*}$-algebras includes the extra axiom that the involution in a $\mathrm{JB}^{*}$-algebra is an isometry (cf. [36, §3.8]). The result in [79, Lemma 4] (see also [16, Proposition 3.3.13]) shows that this extra axiom is redundant. The self-adjoint part of a $\mathrm{JB}^{*}$-algebra $B$ will be denoted by $B_{s a}$. It is known that (real) JB-algebras are precisely the self-adjoint parts of JB*algebras (compare [78]). Any $\mathrm{JB}^{*}$-algebra also admits a structure of a $\mathrm{JB}^{*}$-triple when equipped with the triple product defined by $\{x, y, z\}=\left(x \circ y^{*}\right) \circ z+\left(z \circ y^{*}\right) \circ$ $x-(x \circ z) \circ y^{*}$ in which case $U_{a}(b)=\left\{a, b^{*}, a\right\}$. 
For a general overview of JB-algebras, $\mathrm{JB}^{*}$-algebras and $\mathrm{JB}^{*}$-triples the reader is referred to the monographs [36, 19, 16, 17.

A $J B W^{*}$-triple (respectively, a $J B W^{*}$-algebra) is a $\mathrm{JB}^{*}$-triple (respectively, a $J B^{*}$-algebra) which is also a dual Banach space. JBW*-triples can be considered as the Jordan alter-ego of von Neumann algebras. It should be noted that, as established by Sakai's theorem for von Neumann algebras, every JBW*-triple admits a unique (isometric) predual and its product is separately weak*-to-weak ${ }^{*}$ continuous [8] (see also [17, Theorems 5.7.20 and 5.7.38] for new proofs). These facts rely on the fact proved in [8] (see also [70]) asserting that the bidual of any $\mathrm{JB}^{*}$-triple $E$ is a $\mathrm{JB}^{*}$-triple under a triple product which extends that of $E$ and is separately weak*to-weak* continuous. It is further known that every triple isomorphism between $\mathrm{JBW}^{*}$-triples is automatically weak*-continuous (cf. [40, Corollary 3.22]).

A $J C^{*}$-algebra is a concrete $\mathrm{JB}^{*}$-algebra which materializes as a norm-closed Jordan $*$-subalgebra of a $\mathrm{C}^{*}$-algebra, and hence a norm-closed Jordan self-adjoint subalgebra of some $B(H)$. A JW*-algebra is a $\mathrm{JC}^{*}$-algebra which is also a dual Banach space, or equivalently, a weak*-closed $\mathrm{JB}^{*}$-subalgebra of some von Neumann algebra. There are examples of $\mathrm{JB}^{*}$-algebras which are not $\mathrm{JC}^{*}$-algebras. Macdonald's and Shirshov-Cohn's theorems are useful tools to describe certain important subalgebras of $\mathrm{JB}^{*}$-algebras (see [36, Theorems 2.4.13 and 2.4.14]). For example, these structure results were originally applied by J.D.M. Wright in 78 to deduce that the $\mathrm{JB}^{*}$-subalgebra of a $\mathrm{JB}^{*}$-algebra generated by two hermitian elements (and the unit element) is a $\mathrm{JC}^{*}$-algebra. This result and one of its multiple consequences are gathered in the next lemma (which is a variant of [16, Proposition 3.4.6]).

Lemma 3.1. Let $B$ be a unital $J B^{*}$-algebra and $a \in B$ an arbitrary element.

(i) Let $N$ be the closed Jordan *-subalgebra of $B$ generated by $a$ and 1 . Then $N$ is Jordan (isometrically) *-isomorphic to a $J B^{*}$-subalgebra of some $B(H)$, where $H$ is a complex Hilbert space;

(ii) The element $a^{*} \circ a$ is positive in $N$. Moreover, $a^{*} \circ a=0$ if and only if $a=0$.

Proof. ( $i$ ) Note that $N$ coincides with the unital Jordan $*$-subalgebra generated by two self-adjoint elements $\frac{1}{2}\left(a^{*}+a\right)$ and $\frac{1}{2 i}\left(a-a^{*}\right)$. Hence the assertion follows immediately from [78, Corollary 2.2] (see also [16, Proposition 3.4.6]). Statement (ii) follows from $(i)$ since the assertion is clearly true in $B(H)$.

The literature offers a generous collection of structure results for $\mathrm{JB}^{*}$-triples. The Gelfand-Naimark theorem established by Y. Friedman and B. Russo proves that every $\mathrm{JB}^{*}$-triple can be isometrically embedded as a $\mathrm{JB}^{*}$-subtriple of an $\ell_{\infty}$ sum of Cartan factors (see [30, Theorem 1] and details there). A consequence of this fact shows that every $\mathrm{JB}^{*}$-triple is isometrically $\mathrm{JB}^{*}$-triple isomorphic to a $\mathrm{JB}^{*}$-subtriple of a $\mathrm{JB}^{*}$-algebra.

Let us fix some additional notation. Given two von Neumann algebras $A \subseteq B(H)$ and $W \subseteq B(K)$, the algebraic tensor product $A \otimes W$ is canonically embedded into $B(H \otimes K$ ), where $H \otimes K$ is the hilbertian tensor product of $H$ and $K$ (see [77, Definition IV.1.2]). Then the von Neumann tensor product of $A$ and $W$ (denoted by $A \bar{\otimes} W$, , is precisely the von Neumann subalgebra generated by the algebraic tensor product $A \otimes W$ in $B(H \otimes K)$, that is, the weak* closure of $A \otimes W$ in $B(H \otimes K)$ (see [77, $\S I V .5])$. 
Suppose $A$ is a commutative von Neumann algebra and $M$ is a $\mathrm{JBW}^{*}$-subtriple of some $B(H)$. Following the standard notation, we shall write $A \bar{\otimes} M$ for the weak*-closure of the algebraic tensor product $A \otimes M$ in the usual von Neumann tensor product $A \bar{\otimes} B(H)$ of $A$ and $B(H)$. Clearly $A \bar{\otimes} M$ is a $\mathrm{JBW}^{*}$-subtriple of $A \bar{\otimes} B(H)$. It is well known that every Cartan factor $C$ of type $1,2,3$, or 4 is a JBW*-subtriple of some $B(H)$ (compare 41 and section 9 for a more detailed presentation of Cartan factors), and thus, the von Neumann tensor product $A \bar{\otimes} C$ is a $\mathrm{JBW}^{*}$-triple.

The exceptional Cartan factors of type 5 and 6 can not be represented as JB*subtriples of some $B(H)$. Fortunately, these factors are all finite-dimensional. Henceforth, if $C$ denotes a finite-dimensional JB*-triple, $A \bar{\otimes} C$ will stand for the injective tensor product of $A$ and $C$, which is clearly identified with the space $C(\Omega, C)$, of all continuous functions on $\Omega$ with values in $C$ endowed with the pointwise operations and the supremum norm, where $\Omega$ denotes the spectrum of $A$ (cf. 71, p. 49]). This convention is consistent with the definitions in the previous paragraph, because if $C$ is a finite-dimensional Cartan factor which can be also embedded as a $\mathrm{JBW}^{*}$-subtriple of some $B(H)$ both definitions above give the same object (cf. [77, Theorem IV.4.14]).

In the setting of $\mathrm{JBW}^{*}$-triples, a much more precise description than that derived from the Gelfand-Naimark theorem was found by G. Horn and E. Neher in 41, (1.7)], [42, (1.20)], where they proved that every $\mathrm{JBW}^{*}$-triple $M$ writes in the form

$$
M=\left(\bigoplus_{j \in \mathcal{J}} A_{j} \bar{\otimes} C_{j}\right)_{\ell_{\infty}} \oplus_{\ell_{\infty}} H(W, \alpha) \oplus_{\ell_{\infty}} p V,
$$

where each $A_{j}$ is a commutative von Neumann algebra, each $C_{j}$ is a Cartan factor, $W$ and $V$ are continuous von Neumann algebras, $p$ is a projection in $V, \alpha$ is a linear involution on $W$ commuting with *, that is, a linear ${ }^{*}$-antiautomorphism of period 2 on $W$, and $H(W, \alpha)=\{x \in W: \alpha(x)=x\}$.

We conclude this section by the following result originated from [20, Proposition 2] and [69, Theorem D.20].

Proposition 3.2. [17, Proposition 5.10.137] Let $M$ be any $J B W^{*}$-triple. Then $M$ is triple-isomorphic to a weak ${ }^{*}$-closed subtriple of a JBW*-algebra which is 1complemented by a weak*-to-weak* continuous projection.

\section{MAin Results}

Our main result is the following theorem on coincidence of measures of weak non-compactness in preduals of $\mathrm{JBW}^{*}$-triples. It is proved at the end of Section 8 below.

Theorem 4.1. Let $M$ be a $J B W^{*}$-triple and let $A \subseteq M_{*}$ be a bounded set. Then $\omega(A)=\mathrm{wk}_{M_{*}}(A)=\operatorname{wck}_{M_{*}}(A)$.

Since $\mathrm{JBW}^{*}$-algebras and von Neumann algebras are special cases of JBW*triples, the following two corollaries are immediate.

Corollary 4.2. Let $M$ be a $J B W^{*}$-algebra and let $A \subseteq M_{*}$ be a bounded set. Then $\omega(A)=\mathrm{wk}_{M_{*}}(A)=\operatorname{wck}_{M_{*}}(A)$. 
Corollary 4.3. Let $M$ be a von Neuman algebra and let $A \subseteq M_{*}$ be a bounded set. Then $\omega(A)=\mathrm{wk}_{M_{*}}(A)=\operatorname{wck}_{M_{*}}(A)$.

Note that Corollary 4.2 easily implies the main result Theorem 4.1 using Proposition 3.2 and Lemma 2.1. This is in fact the way the main result will be proved in Section 8

We further easily obtain the same results for real variants of the respective structures.

Corollary 4.4. Let $M$ be a real $J B W^{*}$-triple and let $A \subseteq M_{*}$ be a bounded set. Then $\omega(A)=\mathrm{wk}_{M_{*}}(A)=\operatorname{wck}_{M_{*}}(A)$.

Proof. We use the following arguments explained in detail in [11, Section 6].

Let $M$ be a real $\mathrm{JBW}^{*}$-triple. Then there is a $\mathrm{JBW}^{*}$-triple $N$ and a weak*to-weak* ${ }^{*}$ continuous conjugate-linear isometry $\tau: N \rightarrow N$ such that $\tau^{2}=\mathrm{id}_{N}$ and

$$
M=\{x \in N ; x=\tau(x)\}
$$

For each $\varphi \in N_{*}$ define

$$
\tau^{\#}(\varphi)(x)=\overline{\varphi(\tau(x))}, \quad x \in N
$$

Then $\tau^{\#}$ is a conjugate-linear isometry of $N_{*}$ onto $N_{*}$ such that $\left(\tau^{\#}\right)^{2}=\mathrm{id}_{N_{*}}$. Set

$$
N_{*}^{\tau}=\left\{\varphi \in N_{*} ; \tau^{\#}(\varphi)=\varphi\right\} .
$$

Then $N_{*}^{\tau}$ is a closed real-linear subspace of $N_{*}$ and the mapping $\varphi \mapsto \operatorname{Re} \varphi$ is a real-linear isometry of $N_{*}^{\tau}$ onto $M_{*}$.

Hence the formula

$$
P(\varphi)=\frac{1}{2}\left(\varphi+\tau^{\#}(\varphi)\right), \quad \varphi \in N_{*}
$$

defines a real-linear norm-one projection of $N_{*}$ onto $N_{*}^{\tau}$.

If $X$ is a complex Banach space, denote by $X_{R}$ the underlying real space.

Now, by Theorem 4.1 we know that the measures of weak non-compactness coincide for subsets of $N_{*}$. By the discussion at the end of Section 2.5 of [46] the measures of weak non-compactness with respect to $N_{*}$ coincide with those with respect for $\left(N_{*}\right)_{R}$. Finally, $N_{*}^{\tau}$ is 1-complemented in $\left(N_{*}\right)_{R}$, hence Lemma 2.1yields that the measures of weak non-compactness coincide for subsets of $N_{*}^{\tau}$. Since this space is isometric to $M_{*}$, the proof is complete.

Since JBW-algebras are among the examples of a real $\mathrm{JBW}^{*}$-triple, the following corollary follows immediately.

Corollary 4.5. Let $M$ be a JBW-algebra and let $A \subseteq M_{*}$ be a bounded set. Then $\omega(A)=\mathrm{wk}_{M_{*}}(A)=\mathrm{wck}_{M_{*}}(A)$.

The following corollary also is a special case of our previous result, since the self-adjoint part of a von Neumann algebra $M$ is a real $\mathrm{JBW}^{*}$-triple and its predual is formed exactly by the self-adjoint elements of $M_{*}$.

Corollary 4.6. Let $M$ be a von Neuman algebra and let $A \subseteq M_{*, s a}$ (where $M_{*, s a}$ denotes the subspace of $M_{*}$ formed by self-adjoint functionals) be a bounded set. Then $\omega(A)=\operatorname{wk}_{M_{*, s a}}(A)=\operatorname{wck}_{M_{*, s a}}(A)$. 


\section{Subsequence splitting Property}

In [46, Proposition 7.1] a special case of Theorem 4.1 was proved - the equality of measures of weak non-compactness for subsets of $L^{1}(\mu)$ for a finite measure $\mu$. A key role in the proof is played by a variant of Rosenthal's subsequence splitting lemma. This is a motivation to define the following property of Banach spaces which turns out to be closely connected with the equality of measures of weak non-compactness.

Let $X$ be a Banach space. We say that $X$ has

- the subsequence splitting property if for any bounded sequence $\left(x_{n}\right)$ in $X$ and any $\varepsilon>0$ there is a subsequence $\left(x_{n_{k}}\right)$ which can be expressed as $x_{n_{k}}=y_{k}+z_{k}$, where $\left(y_{k}\right)$ is weakly convergent in $X$ and

$$
\left\|\sum_{j=1}^{n} \alpha_{j} z_{j}\right\| \geq(1-\varepsilon) \sum_{j=1}^{n}\left|\alpha_{j}\right|\left\|z_{j}\right\|
$$

for any $n \in \mathbb{N}$ and any choice of scalars $\alpha_{1}, \ldots, \alpha_{n}$;

- the isometric subsequence splitting property if for any bounded sequence $\left(x_{n}\right)$ in $X$ there is a subsequence $\left(x_{n_{k}}\right)$ which can be expressed as $x_{n_{k}}=y_{k}+z_{k}$, where $\left(y_{k}\right)$ is weakly convergent in $X$ and

$$
\left\|\sum_{j=1}^{n} \alpha_{j} z_{j}\right\|=\sum_{j=1}^{n}\left|\alpha_{j}\right|\left\|z_{j}\right\|
$$

for any $n \in \mathbb{N}$ and any choice of scalars $\alpha_{1}, \ldots, \alpha_{n}$.

That $L^{1}([0,1])$ has the isometric subsequence splitting property has been mentioned by Bourgain and Rosenthal in [12] but a forerunner can be found in [47]. There are also splitting versions for $L^{p}$-spaces, $0<p<\infty$ but in this paper we naturally restrict to the case $p=1$ because preduals of $\mathrm{JBW}^{*}$-triples are generalizations of (non-commutative) $L^{1}$-spaces. The generalization of the isometric subsequence splitting property to preduals of von Neumann algebras was proved by Randrianantoanina [66] and, by different methods, by Raynaud and Xu 67]. Then Fernández-Polo, Ramírez and the third mentioned author [27] showed the isometric subsequence splitting property for preduals of $\mathrm{JBW}^{*}$-algebras and the two last named authors for $\mathrm{JBW}^{*}$-triples 64 .

The predual of a $\mathrm{JBW}^{*}$-triple is L-embedded (see [17, Theorem 5.7.36] or 40], [8]). Recall that a Banach space $X$ is called L-embedded if there is a projection $P$ on $X^{* *}$ with image $X$ such that $\left\|x^{* *}\right\|=\left\|P x^{* *}\right\|+\left\|x^{* *}-P x^{* *}\right\|$ for all $x^{* *} \in$ $X^{* *}$; the standard reference for L-embedded spaces is [37, Chapter IV]. An Lembedded Banach space $X$ is weakly sequentially complete [37, Theorem IV.2.2]. Hence if a bounded sequence $\left(x_{n}\right)$ of $X$ does not contain any $\ell^{1}$-subsequence then by Rosenthal's theorem [25, Theorem 5.37] it contains a weak Cauchy subsequence $\left(x_{n_{k}}\right)$ and in this case the splitting property is trivially satisfied with $y_{k}=x_{n_{k}}$ and $z_{k}=0$. The interesting case appears when $\left(x_{n}\right)$ contains an $\ell^{1}$-sequence. This explains why we use Lemma 5.3 which says that the quality of an $\ell^{1}$-sequence (i.e., its James constant, see below) remains invariant, up to a subsequence, under a perturbation by a weak Cauchy sequence.

The aim of this section is to show the following proposition whose main purpose for this paper is part $(b)$. 
Proposition 5.1. Let $X$ be a Banach space. Consider the following possible properties of $X$.

(I) For any bounded set $A \subseteq X$ we have $\operatorname{wck}_{X}(A)=\omega(A)$.

(II) For any bounded set $A \subseteq X$ and any $\varepsilon>0$ there is a countable subset $C \subseteq A$ such that

$$
\omega\left(C^{\prime}\right)>\omega(A)-\varepsilon \text { for any infinite } C^{\prime} \subseteq C .
$$

Then the following assertions are true.

(a) $(I) \Rightarrow(I I)$;

(b) If $X$ has the subsequence splitting property, then $(I I) \Rightarrow(I)$;

(c) If $X$ is L-embedded and enjoys (I), then $X$ has the subsequence splitting property.

Before we pass to the proof of Proposition [5.1 we recall some known facts on $\ell^{1}$ sequences.

To a bounded sequence $\left(x_{n}\right)$ in a Banach space $X$ we associate its 'James constant'

$$
c_{J}\left(x_{n}\right)=\sup c_{m} \quad \text { where the } \quad c_{m}=\inf \left\{\left\|\sum_{n \geq m} \alpha_{n} x_{n}\right\| ; \sum_{n \geq m}\left|\alpha_{n}\right|=1\right\}
$$

form an increasing sequence. If $\left(x_{n}\right)$ is equivalent to the canonical basis of $\ell^{1}$ then $c_{J}\left(x_{n}\right)>0$ and more specifically, $c_{J}\left(x_{n}\right)>0$ if and only if there is an integer $m$ such that $\left(x_{n}\right)_{n \geq m}$ is equivalent to the canonical basis of $\ell^{1}$. The number $c_{J}\left(x_{n}\right)$ may be thought of as the approximately best $l^{1}$-basis constant of $\left(x_{n}\right)$ in the sense that for each $\varepsilon>0$ there is $m \in \mathbb{N}$ such that $\left\|\sum_{n=m}^{\infty} \alpha_{n} x_{n}\right\| \geq(1-\varepsilon) c_{J}\left(x_{n}\right) \sum_{n=m}^{\infty}\left|\alpha_{n}\right|$ for all $\left(\alpha_{n}\right) \in \ell^{1}$, and $c_{J}\left(x_{n}\right)$ cannot be replaced by a strictly greater constant.

Further, a sequence $\left(z_{l}\right)$ will be called a block sequence of $\left(x_{n}\right)$ if there are successive finite sets $A_{l} \subseteq \mathbb{N}$ (i.e., $\max A_{l}<\min A_{l+1}$ for $l \in \mathbb{N}$ ) and a sequence of scalars $\left(\lambda_{n}\right)$ such that for each $l \in \mathbb{N}$ we have

$$
\sum_{k \in A_{l}}\left|\lambda_{k}\right|=1 \text { and } z_{l}=\sum_{k \in A_{l}} \lambda_{k} x_{k}
$$

Lemma 5.2. Let $\left(x_{n}\right)$ be a bounded sequence in a Banach space $X$ such that $c_{J}\left(x_{n}\right)>0$. Then there is a block sequence $\left(z_{l}\right)$ of $\left(x_{n}\right)$ such that $\left\|z_{l}\right\| \rightarrow c_{J}\left(x_{n}\right)$ as $l \rightarrow \infty$ and, moreover,

$$
\left(1-2^{-m}\right) c_{J}\left(x_{n}\right) \sum_{l=m}^{\infty}\left|\alpha_{l}\right| \leq\left\|\sum_{l=m}^{\infty} \alpha_{l} z_{l}\right\| \leq\left(1+2^{-m}\right) c_{J}\left(x_{n}\right) \sum_{l=m}^{\infty}\left|\alpha_{l}\right|
$$

for all $m \in \mathbb{N}$ and all $\left(\alpha_{n}\right) \in \ell^{1}$.

Proof. Set $c=c_{J}\left(x_{n}\right)$ and let $c_{m}$ have the meaning from (3). Since $c_{m} \nearrow c$, we can fix an increasing sequence $\left(k_{m}\right)$ of natural numbers such that $c_{k_{m}}>c\left(1-2^{-m}\right)$.

Next, having in mind that $c_{k}$ is defined as an infimum and $c_{k} \leq c$ for each $k \in \mathbb{N}$, we can find finite sets $A_{m} \subseteq \mathbb{N}$ and constants $\lambda_{n}, n \in A_{m}$, such that for each $m \in \mathbb{N}$ we have

- $k_{m} \leq \min A_{m} \leq \max A_{m}<\min A_{m+1}$,

- $\sum_{n \in A_{m}}\left|\lambda_{n}\right|=1$,

- $\left\|\sum_{n \in A_{m}} \lambda_{n} x_{n}\right\|<c\left(1+2^{-m}\right)$. 
Set $z_{m}=\sum_{n \in A_{m}} \lambda_{n} x_{n}$. Then clearly $\left(z_{m}\right)$ is a block sequence of $\left(x_{n}\right)$ with $\left\|z_{m}\right\| \rightarrow$ $c$ as $m \rightarrow \infty$. Moreover, fix any sequence $\left(\alpha_{l}\right) \in \ell^{1}$ and $m \in \mathbb{N}$. Then

$$
\left\|\sum_{l=m}^{\infty} \alpha_{l} z_{l}\right\| \leq \sum_{l=m}^{\infty}\left|\alpha_{l}\right|\left\|z_{l}\right\| \leq\left(1+2^{-m}\right) c \sum_{l=m}^{\infty}\left|\alpha_{l}\right|,
$$

and

$$
\begin{aligned}
\left\|\sum_{l=m}^{\infty} \alpha_{l} z_{l}\right\| & =\left\|\sum_{l=m}^{\infty} \sum_{n \in A_{l}} \alpha_{l} \lambda_{n} x_{n}\right\| \geq\left(1-2^{-m}\right) c \sum_{l=m}^{\infty} \sum_{n \in A_{l}}\left|\alpha_{l}\right| \cdot\left|\lambda_{n}\right| \\
& =\left(1-2^{-m}\right) c \sum_{l=m}^{\infty}\left|\alpha_{l}\right|,
\end{aligned}
$$

which completes the proof.

It is elementary but useful that if one passes to a subsequence $\left(x_{n_{k}}\right)$ of $\left(x_{n}\right)$ then $c_{J}\left(x_{n_{k}}\right) \geq c_{J}\left(x_{n}\right)$; in particular it makes sense to define

$$
\tilde{c}_{J}\left(x_{n}\right)=\sup _{n_{k}} c_{J}\left(x_{n_{k}}\right) .
$$

A diagonal argument shows that every bounded sequence $\left(x_{n}\right)$ admits a subsequence $\left(x_{n_{k}}\right)$ which is $c_{J}$-stable in the sense that $c_{J}\left(x_{n_{k}}\right)=\tilde{c}_{J}\left(x_{n_{k}}\right)$. If one passes to a block sequence $\left(z_{n}\right)$ of $\left(x_{n}\right)$ then $c_{J}\left(z_{n}\right) \geq c_{J}\left(x_{n}\right)$.

Lemma 5.3. Let $\left(x_{n}\right)$ be a bounded sequence in a Banach space $X$. Let $\left(y_{n}\right)$ be a weak Cauchy sequence in $X$. Then for each $\varepsilon \in(0,1)$ there is a subsequence $\left(n_{k}\right)$ such that $\left(x_{n_{k}}\right)$ and $\left(x_{n_{k}}+y_{n_{k}}\right)$ are $c_{J}$-stable and

$$
\begin{array}{r}
(1-\varepsilon) \tilde{c}_{J}\left(x_{n}\right) \leq c_{J}\left(x_{n_{k}}\right) \leq \tilde{c}_{J}\left(x_{n}\right) . \\
c_{J}\left(x_{n_{k}}+y_{n_{k}}\right)=c_{J}\left(x_{n_{k}}\right) .
\end{array}
$$

Proof. Let $\left(x_{n}\right)$ be a bounded sequence. If $\tilde{c}_{J}\left(x_{n}\right)=0$ then by Rosenthal's $\ell^{1}$ theorem $\left(x_{n}\right)$ contains a weak Cauchy subsequence which remains weak Cauchy when a weak Cauchy sequence is added; hence the $c_{J}$-value of the sum is still 0 and we are done in this case.

Assume now that $\tilde{c}_{J}\left(x_{n}\right)>0$. Let $\left(y_{n}\right)$ be weakly Cauchy and let $0<\varepsilon<1$. Set $z_{n}=x_{n}+y_{n}$. Choose a subsequence $\left(x_{n_{k}}\right)$ such that (6) holds. Passing to another subsequence, if necessary, we assume further that $\left(x_{n_{k}}\right)$ and $\left(z_{n_{k}}\right)$ are $c_{J}$-stable. Write $c=c_{J}\left(x_{n_{k}}\right)$ for short. Note that $c \geq(1-\varepsilon) \tilde{c}_{J}\left(x_{n}\right)>0$. In particular we may assume that $\left(x_{n_{k}}\right)$ is an $\ell^{1}$-sequence (by omitting, if necessary, finitely many $x_{n_{k}}$ ). Passing to another subsequence again, if necessary, we also get that $c_{J}\left(z_{n_{k}}\right)>0$ because otherwise $\left(z_{n_{k}}\right)$ would have no $\ell^{1}$-subsequence and would therefore contain a weak Cauchy subsequence $\left(z_{n_{k_{l}}}\right)$ by Rosenthal's theorem in which case $x_{n_{k_{l}}}=z_{n_{k_{l}}}-y_{n_{k_{l}}}$ would form a weak Cauchy sequence which is not possible. By Lemma 5.2 take blocks $x_{n}^{(1)}$ of the sequence $\left(x_{n_{k}}\right)$ such that

$$
\left(1-2^{-m}\right) c \sum_{l=m}^{\infty}\left|\alpha_{l}\right| \leq\left\|\sum_{l=m}^{\infty} \alpha_{l} x_{l}^{(1)}\right\| \leq\left(1+2^{-m}\right) c \sum_{l=m}^{\infty}\left|\alpha_{l}\right|
$$


for all $m \in \mathbb{N}$ and $\left(\alpha_{l}\right) \in \ell^{1}$. For the corresponding blocks $z_{n}^{(1)}$ we have $c_{J}\left(z_{n}^{(1)}\right) \geq$ $c_{J}\left(z_{n_{k}}\right)>0$. By Lemma 5.2 take blocks $z_{n}^{(2)}$ of $\left(z_{n}^{(1)}\right)$ such that

$$
\left(1-2^{-m}\right) c_{J}\left(z_{n}^{(1)}\right) \sum_{l=m}^{\infty}\left|\alpha_{l}\right| \leq\left\|\sum_{l=m}^{\infty} \alpha_{l} z_{l}^{(2)}\right\| \leq\left(1+2^{-m}\right) c_{J}\left(z_{n}^{(1)}\right) \sum_{l=m}^{\infty}\left|\alpha_{l}\right|
$$

for all $m \in \mathbb{N}$ and $\left(\alpha_{l}\right) \in \ell^{1}$. Note that every block sequence of $\left(y_{n}\right)$ admits a weak Cauchy subsequence [for, if $u_{n}=\sum_{k \in A_{n}} \lambda_{k} y_{k}$, (with finite pairwise disjoint $A_{n}$, $\left.\sum_{k \in A_{n}}\left|\lambda_{k}\right|=1\right)$ and if $\left(u_{n_{m}}\right)$ is a subsequence such that $\lambda=\lim _{m} \sum_{k \in A_{n_{m}}} \lambda_{k}$ exists then, given $x^{*} \in X^{*}$, the sequence $x^{*}\left(u_{n_{m}}\right)$ converges to $\lambda \mu$ where $\mu=$ $\lim _{n} x^{*}\left(y_{n}\right):\left|x^{*}\left(u_{n_{m}}\right)-\lambda \mu\right|=\left|\sum_{k \in A_{n_{m}}} \lambda_{k}\left(x^{*}\left(y_{k}\right)-\mu\right)+\left(\left(\sum_{k \in A_{n_{m}}} \lambda_{k}\right)-\lambda\right) \mu\right| \leq$ $\max _{k \in A_{n_{m}}}\left|x^{*}\left(y_{k}\right)-\mu\right|+\left|\left(\sum_{k \in A_{n_{m}}} \lambda_{k}\right)-\lambda\right||\mu| \rightarrow 0$ as $\left.m \rightarrow \infty\right]$. Hence the blocks $y_{n}^{(2)}$ which correspond to $z_{n}^{(2)}$ admit a weak Cauchy subsequence $\left(y_{n_{k}}^{(2)}\right)$ whose differences $\frac{1}{2}\left(y_{n_{2 k+1}}^{(2)}-y_{n_{2 k}}^{(2)}\right)$ are weakly null and, by the Mazur theorem, there are blocks $y_{n}^{(3)}$ of $\left(y_{n}^{(2)}\right)$ such that $\left\|y_{n}^{(3)}\right\| \rightarrow 0$. Note that for the corresponding blocks $x_{n}^{(3)}$ (which are blocks of the $x_{n}^{(1)}$ ) we have $\left\|x_{n}^{(3)}\right\| \rightarrow c$ by (8). Hence the norm of the right hand side in

$$
z_{n}^{(3)}=x_{n}^{(3)}+y_{n}^{(3)}
$$

converges to $c$ while the norm of the left hand side converges to $c_{J}\left(z_{n}^{(1)}\right)$ by (9). Thus $c=c_{J}\left(z_{n}^{(1)}\right)$. This shows that $c_{J}\left(x_{n_{k}}\right)=c_{J}\left(z_{n}^{(1)}\right) \geq c_{J}\left(x_{n_{k}}+y_{n_{k}}\right)$.

Apply now what has been shown so far to the bounded sequence $\left(z_{n_{k}}\right)$ and the weak Cauchy sequence $\left(-y_{n_{k}}\right)$ in order to obtain subsequences $\left(z_{n_{k_{l}}}\right)$ and $\left(y_{n_{k_{l}}}\right)$ such that, by $c_{J}$-stability of $\left(x_{n_{k}}\right)$ and $\left(z_{n_{k}}\right)$,

$$
c_{J}\left(x_{n_{k}}\right)=c_{J}\left(x_{n_{k_{l}}}\right)=c_{J}\left(z_{n_{k_{l}}}+\left(-y_{n_{k_{l}}}\right)\right) \leq c_{J}\left(z_{n_{k_{l}}}\right)=c_{J}\left(z_{n_{k}}\right) .
$$

Now (7) follows.

Proof of Proposition 5.1. (a) The proof of the implication (I) $\Rightarrow$ (II) is almost immediate from the definition of $\operatorname{wck}_{X}$. For, given $\varepsilon>0$, it is enough to set $C=\left\{x_{n}\right\}$ where $\left(x_{n}\right)$ is a sequence in $A$ such that $\operatorname{dist}\left(x^{* *}, X\right)>\operatorname{wck}_{X}(A)-\varepsilon$ for all weak*cluster points $x^{* *}$ of $\left(x_{n}\right)$ which leads to $\omega\left(C^{\prime}\right)>\operatorname{wck}_{X}(A)-\varepsilon=\omega(A)-\varepsilon$ for all infinite $C^{\prime} \subseteq C$.

(b) Suppose that $X$ has the subsequence splitting property and (II) is satisfied. Let $A$ be bounded in $X$. We need to prove that $\operatorname{wck}_{X}(A) \geq \omega(A)$. If $\omega(A)=0$, the inequality is trivial. So, assume $\omega(A)>0$ and fix an arbitrary $\varepsilon \in(0, \min \{1, \omega(A)\})$. Let $C=\left\{x_{n}\right\}$ be a countable subset of $A$ provided by (II). In particular, $\omega\left(\left\{x_{n_{k}}\right\}\right)>$ 0 for every subsequence $\left(x_{n_{k}}\right)$ of $\left(x_{n}\right)$.

Take a subsequence $\left(x_{n_{k}}\right)$ of $\left(x_{n}\right)$ such that $\tilde{c}_{J}\left(x_{n}\right)-\varepsilon<c_{J}\left(x_{n_{k}}\right) \leq \tilde{c}_{J}\left(x_{n}\right)$. Take another subsequence (still denoted by $\left(x_{n_{k}}\right)$ ) and write $x_{n_{k}}=y_{k}+z_{k}$ according to the subsequence splitting property where $\left(y_{k}\right)$ converges weakly and $\left\|\sum \alpha_{k} z_{k}\right\| \geq$ $(1-\varepsilon / 2) \sum\left|\alpha_{k}\right|\left\|z_{k}\right\|$ for all $\left(\alpha_{k}\right) \in \ell^{1}$. Note that the sequence $\left(z_{k}\right)$ is bounded, so without loss of generality $\lambda:=\lim \left\|z_{k}\right\|$ exists. We have that $\lambda>0$ because otherwise $\left(x_{n_{k}}\right)$ would be weakly convergent which would contradict $\omega\left(\left\{x_{n_{k}}\right\}\right)>0$. Hence, without loss of generality $\lambda(1-\varepsilon / 2)<\left\|z_{k}\right\|<\lambda(1+\varepsilon)$ for $k \in \mathbb{N}$. Since the 
sequence $\left(y_{k}\right)$ forms a relatively weakly compact set, by the assumptions we have

$$
\omega(A)-\varepsilon<\omega\left(\left\{x_{n_{k}}, k \in \mathbb{N}\right\}\right) \leq \sup _{k}\left\|z_{k}\right\| \leq \lambda(1+\varepsilon) .
$$

Moreover, $c_{J}\left(z_{k}\right) \geq(1-\varepsilon / 2) \lambda(1-\varepsilon / 2)>(1-\varepsilon) \lambda$.

We apply Lemma 5.3 and obtain further subsequences (still denoted by $\left(x_{n_{k}}\right)$, $\left.\left(y_{k}\right),\left(z_{k}\right)\right)$. Then (7) yields

$$
c_{J}\left(x_{n_{k}}\right)=c_{J}\left(z_{k}\right) \geq \lambda(1-\varepsilon) \geq \frac{1-\varepsilon}{1+\varepsilon}(\omega(A)-\varepsilon) .
$$

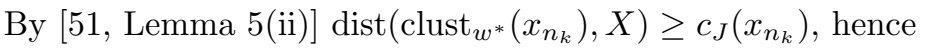

$$
\operatorname{wck}_{X}(A) \geq \operatorname{dist}\left(\operatorname{clust}_{w^{*}}\left(x_{n_{k}}\right), X\right) \geq c_{J}\left(x_{n_{k}}\right) \geq \frac{1-\varepsilon}{1+\varepsilon}(\omega(A)-\varepsilon) .
$$

Since $\varepsilon$ is arbitrary we are done.

(c) Assume $X$ is $L$-embedded and satisfies (I). Fix a bounded sequence $\left(x_{n}\right)$ in $X$. If $\tilde{c}_{J}\left(x_{n}\right)=0$ then by Rosenthal's $\ell^{1}$-theorem, $\left(x_{n}\right)$ contains a weak Cauchy subsequence $\left(x_{n_{k}}\right)$ which, by weak sequential completeness of L-embedded spaces 37. Theorem IV.2.2] converges weakly. The case is settled by putting $y_{k}=x_{n_{k}}$ and $z_{k}=0$.

Let us now suppose that $\tilde{c}:=\tilde{c}_{J}\left(x_{n}\right)>0$. In order to prove the subsequence splitting property in this case it is enough to produce, given $\varepsilon>0$, a decomposition $x_{n_{k}}=y_{k}+z_{k}$ where $\left(y_{k}\right)$ converges weakly and where

$$
(1-\varepsilon) \tilde{c} \sum\left|\alpha_{k}\right| \leq\left\|\sum \alpha_{k} z_{k}\right\| \leq(1+\varepsilon) \tilde{c} \sum\left|\alpha_{k}\right|
$$

for all $\left(\alpha_{k}\right) \in \ell^{1}$. Set $A=\left\{x_{n} ; n \in \mathbb{N}\right\}$.

First we claim that $\operatorname{wck}_{X}(A)=\tilde{c}$. The inequality " $\geq$ " follows from 51, Lemma $5(\mathrm{ii})]$. For the other inequality note that for any $\eta>0$, the construction (which works also for complex scalars) leading to [51, formula (8)] yields an $x \in X$ and a subsequence $\left(x_{n_{k}}\right)$ such that $c_{J}\left(x_{n_{k}}-x\right)>(1-\eta) \operatorname{wck}_{X}(A)$. (Note that here the assumption that $X$ is $L$-embedded is used). It follows $c_{J}\left(x_{n_{k}}\right)>(1-\eta) \operatorname{wck}_{X}(A)$ (cf. [56, Proposition 4.2] or Lemma 5.3 for constant $y_{n}=-x$ ). This proves " $\leq$ " and the claim.

Let $\left(x_{n_{k}}\right)$ be a subsequence such that $c_{J}\left(x_{n_{k}}\right)>\left(1-\frac{\varepsilon}{2}\right) \tilde{c}$. Since $\omega(A)=\tilde{c}$ by (I) and the claim, there is a weakly compact set $K$ of $X$ such that $A \subseteq K+(1+\varepsilon) \tilde{c} B_{X}$. Choose a sequence $\left(y_{k}\right)$ in $K$ (which can be supposed to converge weakly) such that $x_{n_{k}}=y_{k}+z_{k}$ with $\left\|z_{k}\right\| \leq(1+\varepsilon) \tilde{c}$. The latter inequality implies the second one of (10). By Lemma 5.3 we pass to subsequences (still denoted by $\left.\left(x_{n_{k}}\right),\left(y_{k}\right),\left(z_{k}\right)\right)$ in order to get $c_{J}\left(z_{k}\right)=c_{J}\left(x_{n_{k}}\right)$. Now, by the definition of $c_{J}\left(z_{k}\right)$, if we omit at most finitely many $z_{k}$ then we have

$$
\left\|\sum \alpha_{k} z_{k}\right\| \geq\left(1-\frac{\varepsilon}{2}\right) c_{J}\left(z_{k}\right) \sum\left|\alpha_{k}\right| \geq\left(1-\frac{\varepsilon}{2}\right)^{2} \tilde{c} \sum\left|\alpha_{k}\right|
$$

and the first inequality of (10) follows.

Proposition $5.1(b)$ will play a key role in the proof of Theorem 4.1 in Section 8 Let us remark that some special cases may be proved already now, as there are some spaces which are easily seen to satisfy (II). 
Recall that a Banach space $X$ is said to be weakly compactly generated (shortly $W C G)$ if there is a weakly compact subset $K \subseteq X$ with $\overline{\operatorname{span}} K=X$. Further, $X$ is called strongly $W C G$ (see [73]) if there is a weakly compact set $K \subseteq X$ such that

$$
\forall L \subseteq X \text { weakly compact } \forall \varepsilon>0 \exists n \in \mathbb{N}: L \subseteq n K+\varepsilon B_{X} .
$$

By the Krein theorem we may assume without loss of generality that $K$ is absolutely convex.

We have the following easy lemma

Lemma 5.4. Let $X$ be a strongly WCG Banach space. Then for any bounded set $A \subseteq X$ there is a countable subset $C \subseteq A$ such that $\omega\left(C^{\prime}\right)=\omega(A)$ for each $C^{\prime} \subseteq C$ infinite.

Proof. Let $K \subseteq X$ be a weakly compact set witnessing the strong WCG property. Then clearly

$$
\omega(A)=\inf _{n \in \mathbb{N}} \widehat{\mathrm{d}}(A, n K)
$$

for any bounded set $A \subseteq X$. Given any bounded set $A \subseteq X$, we can find a sequence $\left(x_{n}\right)$ in $A$ such that $\operatorname{dist}\left(x_{n}, n K\right)>\omega(A)-\frac{1}{n}$. It is enough to take $C=\left\{x_{n} ; n \in \mathbb{N}\right\}$.

Since $L^{1}(\mu)$ is strongly WCG for any finite measure $\mu$ (by [73, Example 2.3(d)]) and $L^{1}$ spaces have the subsequence splitting property, then the essential part of [46. Proposition 7.1] follows immediately from the previous lemma and Proposition 5.1]

Further, preduals of $\sigma$-finite von Neumann algebras and, more generally, preduals of $\sigma$-finite $\mathrm{JBW}^{*}$-algebras are seen to be strongly WCG by combining 73 , Theorem 2.1], [43, Appendice 6, Lemma 2] (cf. Lemma 7.3 below), and 69, Theorem D.21] (see Proposition 7.9 below). Hence the validity of Theorem 4.1 for $\sigma$-finite $\mathrm{JBW}^{*}$ algebras easily follows. The case of $\sigma$-finite $\mathrm{JBW}^{*}$-triples is more complicated, see Theorem 9.3 below.

\section{TRIPOTENTS AND PROJECTIONS}

A projection in a $C^{*}$-algebra $A$ is a self-adjoint idempotent, i.e., an element $p \in A$ satisfying $p^{*}=p=p^{2}$. If $A$ is represented as a $C^{*}$-subalgebra of $B(H)$, then $p \in A$ is a projection if and only if it is, when viewed as an operator on $H$, an orthogonal projection.

Similarly, a projection in a $\mathrm{JB}^{*}$-algebra $A$ is an element $p \in A$ satisfying $p^{*}=p$ and $p \circ p=p$; in particular, $p$ is positive (by Lemma 3.1). Two projections $p, q \in A$ are said to be orthogonal if $p \circ q=0$ or, equivalently, if $p+q$ is also a projection. In case $A$ is a $C^{*}$-algebra, this is just equivalent to $p q=0$, which means that the ranges of the projections are orthogonal subspaces of $H$.

We shall consider the usual partial order on the set of projections in a $\mathrm{JB}^{*}$-algebra defined by $p \leq q$ if $q-p$ is a projection. In a $C^{*}$-algebra this order coincides with the standard one.

In a $\mathrm{JB}^{*}$-triple there is no natural notion of projections, but tripotents play a similar role. As a motivation for the latter notion, suppose $A$ is a $\mathrm{C}^{*}$-algebra regarded as a $\mathrm{JB}^{*}$-triple with respect to the triple product $\{a, b, c\}=\frac{1}{2}\left(a b^{*} c+c b^{*} a\right)$. It is well known that an element $u$ in $A$ is a partial isometry (i.e., $u^{*} u$ is a projection, or equivalently, $u u^{*}$ is a projection) if and only if $\{u, u, u\}=u u^{*} u=u$. Given a 
partial isometry $u$ in a $\mathrm{C}^{*}$-algebra $A$, the elements $p_{i}=u^{*} u$ and $p_{f}=u u^{*}$ are called the initial and final projection of $u$, respectively. An element $u$ of a $\mathrm{JB}^{*}$-triple $M$ is called a tripotent if $\{u, u, u\}=u$. If $M$ is a $\mathrm{JB}^{*}$-algebra, then $u \in M$ is a tripotent if and only if

$$
u=2\left(u \circ u^{*}\right) \circ u-(u \circ u) \circ u^{*},
$$

which cannot be simplified. Note that the projections of a JB*-algebra $A$ are precisely those tripotents in $A$ which are positive elements.

6.1. Peirce decomposition. From a purely algebraic point of view, a complex linear space $E$ equipped with a triple product $\{., .,\}:. E^{3} \rightarrow E$, which is symmetric and bilinear in the outer variables and conjugate-linear in the middle one satisfying the axiom $\left(J B^{*}-1\right)$ in the definition of $\mathrm{JB}^{*}$-triple is called a complex Jordan triple system. An element $u$ in a Jordan triple system $E$ is a tripotent if $\{u, u, u\}=u$. Each tripotent $u$ in a complex Jordan triple system $E$ induces a decomposition of $E$ in terms of the eigenspaces of the mapping $L(e, e)$ (this purely algebraic result can be seen, for example, in [58, 1.3 in page 7] or [19, page 32]).

In our concrete setting, the algebraic structure assures that for each tripotent $u$ in a $\mathrm{JB}^{*}$-triple $M$, the eigenvalues of the operator $L(u, u)$ are contained in the set $\left\{0, \frac{1}{2}, 1\right\}$. If $u \neq 0$, then 1 is always an eigenvalue, the witnessing eigenvector is $u$. For $j=0,1,2$ we shall denote by $M_{j}(u)$ the eigenspace of $M$ with respect to the eigenvalue $\frac{j}{2}$. Then $M$ is the direct sum $M_{0}(u) \oplus M_{1}(u) \oplus M_{2}(u)$ of the three eigenspaces of $L(u, u)$; this decomposition is called the Peirce decomposition of $M$ with respect to $u$. The canonical projection, $P_{j}(u)$, of $M$ onto $M_{j}(u)$ is called the $(j$-)Peirce projection associated with $u$. Peirce projections are explicitly determined by the following formulae:

$$
\begin{aligned}
& P_{2}(u)=L(u, u)\left(2 L(u, u)-\operatorname{id}_{M}\right)=Q(u)^{2}, \\
& P_{1}(u)=4 L(u, u)\left(\operatorname{id}_{M}-L(u, u)\right)=2\left(L(u, u)-Q(u)^{2}\right), \\
& P_{0}(u)=\left(\operatorname{id}_{M}-L(u, u)\right)\left(\operatorname{id}_{M}-2 L(u, u)\right)=\operatorname{id}_{M}-2 L(u, u)+Q(u)^{2}
\end{aligned}
$$

where the quadratic operator $Q(u): M \rightarrow M$ is defined by $Q(u)(x)=\{u, x, u\}$ (compare [58, 1.3 in page 7] or [19, page 7]). In the setting of JB*-triples, Peirce projections are all contractive (see [29, Corollary 1.2] or [19, 3.2.1]).

In case $M$ is a $C^{*}$-algebra and $u \in M$ is a tripotent (i.e., a partial isometry with initial projection $p_{i}$ and final projection $p_{f}$ ), the Peirce projections are given by the following expressions:

$P_{2}(u) x=p_{f} x p_{i}, \quad P_{1}(u) x=p_{f} x\left(1-p_{i}\right)+\left(1-p_{f}\right) x p_{i}, \quad P_{0}(u) x=\left(1-p_{f}\right) x\left(1-p_{i}\right)$,

where $x$ runs through $M$.

If $i, j, k \in\{0,1,2\}$, then the so-called Peirce arithmetic or Peirce multiplication rules say that

$$
\begin{cases}\left\{M_{i}(u), M_{j}(u), M_{k}(u)\right\} \subseteq M_{i-j+k}(u), & \text { if } i-j+k \in\{0,1,2\}, \\ \left\{M_{i}(u), M_{j}(u), M_{k}(u)\right\}=0, & \text { if } i-j+k \notin\{0,1,2\}, \\ \left\{M_{2}(u), M_{0}(u), M\right\}=\left\{M_{0}(u), M_{2}(u), M\right\}=0, & \end{cases}
$$

(see [58, (1.20)-(1.22) in pages 7-8] or [19, Theorem 1.2.44]).

It follows immediately from the Peirce multiplication rules that $M_{j}(u)$ is a JB*subtriple for $j=0,1,2$. In the case $j=2$ something more can be said. In this 
case $M_{2}(u)$ is even a unital $\mathrm{JB}^{*}$-algebra with respect to the product and involution given by

$$
a \circ b=\{a, u, b\}, \quad a^{*}=\{u, a, u\}, \quad a, b \in M_{2}(u),
$$

respectively (cf.[19, $\S 1.2$ and Remark 3.2.2] or [16, Corollary 4.2.30]). It is further known that $u$ is the unit of this $\mathrm{JB}^{*}$-algebra. (If we wish to stress that the operations are with respect to $u$, we write $a \circ_{u} b$ and $a^{* u}$.)

6.2. Complete tripotents. A tripotent $u$ in a $\mathrm{JB}^{*}$-triple $M$ is called complete if $M_{0}(u)=\{0\}$ and unitary if $M=M_{2}(u)$ (that is if $\{u, u, x\}=x$ for all $x \in M$ ). It follows from the above structure results that $M$ admits a unitary tripotent if and only if it admits a structure of unital JB*-algebra (cf. [16, Theorem 4.1.55]). Further, if $M$ is a unital $\mathrm{JB}^{*}$-algebra, then $u \in M$ is a unitary tripotent if and only if it is a unitary element, i.e., an element satisfying that $u$ is invertible in the Jordan sense (i.e., there exists a unique element $b=u^{-1}$ in $M$ such that $b \circ u=1$ and $u^{2} \circ b=u$ ) and $u^{-1}=u^{*}$ (compare [16, §4.1.1]). In a $C^{*}$-algebra this reduces to $u^{*} u=u u^{*}=1$.

A complete tripotent need not be unitary, even in the $C^{*}$-algebra case. Indeed, if $u \in B(H)$ is a partial isometry whose initial projection is the identity on $H$ but its final projection is strictly smaller than the identity on $H$ (or vice versa), i.e., if $u^{*} u=1 \neq u u^{*}$ (or vice versa), then $u$ is a complete non-unitary tripotent. This is not the unique possibility, but it is an important case as witnessed by the forthcoming lemmata. We observe that the extreme points of the closed unit ball of a $\mathrm{C}^{*}$-algebra $A$ are precisely the complete partial isometries (tripotents) of $A$ (see [48, Theorem 1] or [77, Theorem I.10.2]). The same result remains true for the closed unit ball of a $\mathrm{JB}^{*}$-triple $E$, that is, the complete tripotents in $E$ are the extreme points of its closed unit ball (cf. [13, Lemma 4.1] and [55, Proposition 3.5] or [19, Theorem 3.2.3]).

We recall that a conjugation on a complex Banach space $X$ is a conjugate-linear isometry $\tau: X \rightarrow X$ of period-2 (i.e., $\tau^{2}=I d_{X}$ ). Let $H$ be a complex Hilbert space, and let us fix an orthonormal basis $\left(\xi_{k}\right)_{k \in \Lambda}$ in $H$. Given $\xi \in H$, let $\tau(\xi) \in H$ be the vector defined by $\tau(\xi)=\sum_{k \in \Lambda} \overline{\left\langle\xi, \xi_{k}\right\rangle} \xi_{k}$. Then $\tau$ is a conjugation on $H$ and, moreover, any conjugation is of that form (with a properly chosen orthonormal basis).

Lemma 6.1. Let $M$ be a unital $C^{*}$-algebra, and let $u \in M$ be a complete tripotent. Then there exist a complex Hilbert space $H$ and an isometric unital Jordan *monomorphism $\psi: M \rightarrow B(H)$ such that $\psi(u)^{*} \psi(u)=1$.

Proof. By applying the Gelfand-Naimark-Segal construction, we can find a family of complex Hilbert spaces $\left\{H_{i}\right\}_{i \in I}$ and irreducible representations $\Phi_{i}: M \rightarrow B\left(H_{i}\right)$, such that

$$
\Phi=\bigoplus_{i \in I} \Phi_{i}: M \rightarrow \bigoplus_{i \in I}^{\ell_{\infty}} B\left(H_{i}\right) \subseteq B\left(\bigoplus_{i \in I}^{\ell_{2}} H_{i}\right)
$$

is an isometric *-monomorphism (we can consider, for example, the atomic representation of $M$ [59, 4.3.7], where the family $I$ is precisely the set of all pure states of $M$ and each $\Phi_{i}$ is the irreducible representation associated with the pure state $i$ [59, Theorem 3.13.2]). 
Fix $i \in I$. Since $u$ is tripotent in $M, \Phi_{i}(u)$ is a tripotent in $\Phi_{i}(M)$ as well. Moreover, $u$ is complete, i.e.,

$$
\left(1-u u^{*}\right) a\left(1-u^{*} u\right)=0 \text { for } a \in M
$$

hence

$$
\left(1-\Phi_{i}(u) \Phi_{i}(u)^{*}\right) x\left(1-\Phi_{i}(u)^{*} \Phi_{i}(u)\right)=0 \text { for } x \in \Phi_{i}(M) .
$$

Since $\Phi_{i}$ is irreducible, its range is weak*-dense in $B\left(H_{i}\right)$, thus the above formula holds for all $x \in B\left(H_{i}\right)$. In other words, $\Phi_{i}(u)$ is a complete tripotent in $B\left(H_{i}\right)$. Having in mind that $B\left(H_{i}\right)$ is a factor, it follows that $\Phi_{i}(u)^{*} \Phi_{i}(u)=1$ or $\Phi_{i}(u) \Phi_{i}(u)^{*}=1$ (this follows e.g. from [77, Lemma V.1.7] applied to the projections $1-\Phi_{i}(u)^{*} \Phi_{i}(u)$ and $\left.1-\Phi_{i}(u) \Phi_{i}(u)^{*}\right)$.

Let $I_{1}:=\left\{j \in I: \Phi_{j}(u)^{*} \Phi_{j}(u)=1_{j}\right\}$ and $I_{2}:=I \backslash I_{1}$.

For each $j \in I_{2}$, we can find a ${ }^{*}$-anti-homomorphism $\Psi_{j}: B\left(H_{j}\right) \rightarrow B\left(H_{j}\right)$ (consider, for example a transposition on $B\left(H_{j}\right)$ defined by $\Psi_{j}(a):=\tau a^{*} \tau$, where $\tau$ is the conjugation on $H_{j}$ described before the statement of the lemma). For $j \in I_{1}$, $\Psi_{j}$ will stand for the identity on $B\left(H_{j}\right)$. Let $\Psi=\bigoplus_{j \in I} \Psi_{j}: \bigoplus_{j}^{\ell_{\infty}} B\left(H_{j}\right) \rightarrow$ $\bigoplus_{j}^{\ell_{\infty}} B\left(H_{j}\right)$. Clearly $\Psi$ is a Jordan ${ }^{*}$-isomorphism. By construction we have $\Psi(\Phi(u))^{*} \Psi(\Phi(u))=1$, the identity in $\bigoplus_{j}^{\ell_{\infty}} B\left(H_{j}\right)$. Finally, we can embed the $\mathrm{C}^{*}$ algebra $\bigoplus_{j}^{\ell_{\infty}} B\left(H_{j}\right)$ inside $B\left(\bigoplus_{j}^{\ell_{2}} H_{j}\right)$ via a ${ }^{*}$-monomorphism $\theta$, and the Jordan ${ }^{*}$-monomorphism $\psi=\theta \circ \Psi \circ \Phi: M \rightarrow B\left(\bigoplus_{j}^{\ell_{2}} H_{j}\right)$ satisfies the desired property.

We continue with a technical result relating complete tripotents in a $\mathrm{JC}^{*}$-algebra $A$ with the complete tripotents in the $\mathrm{C}^{*}$-algebra generated by $A$.

Lemma 6.2. Let $M$ be a unital $J B^{*}$-algebra. Let $u$ be a complete tripotent in $M$, and let $N$ denote the $J B^{*}$-subalgebra of $M$ generated by $u$ and the unit element. Then $N$ is a $J C^{*}$-subalgebra of some $C^{*}$-algebra $B$, and $u$ is a complete tripotent in the $C^{*}$-subalgebra of $B$ generated by $N$.

Proof. The first statement follows from Lemma $3.1(i)$, so fix a $C^{*}$-algebra $B$ conitaing $N$ as a $\mathrm{JC}^{*}$-subalgebra. Let $A$ be the $\mathrm{C}^{*}$-subalgebra of $B$ generated by $N$. Furhter, let 1 denote the unit of $M$ (which belongs to $N$ ). Then for any $x \in N$ we have $1 x 1=\{1, x, 1\}=x$, hence $x=1 x=x 1$. Since $A$ is generated by $N$, it follows that 1 is the unit of $A$.

Clearly $u$ is a tripotent (hence a partial isometry) in $A$, so its Peirce-0 projection is given by

$$
P_{0}(u)(a)=\left(1-u u^{*}\right) a\left(1-u^{*} u\right), \quad a \in A .
$$

To prove that $u$ is complete in $A$ it is enough to show that $P_{0}(u)$ vanishes on all the (associative) monomials in $u$ and $u^{*}$. To this end, we will consider the formal degree of such monomials in the obvious way ( 1 is the unique monomial of degree 0 , monomials of degree 1 are $u$ and $u^{*}$, monomials of degree 2 are $u^{2},\left(u^{*}\right)^{2}, u u^{*}$ and $u^{*} u$ etc.).

Since $u$ is complete in $N$ and $1, u, u^{*} \in N$, we deduce that $P_{0}(u)$ vanishes on monomials of degree 0 or 1 . Assume that $n \in \mathbb{N}$ and $P_{0}(u)$ vanishes on all the monomials of degree at most $n$. Let $a$ be a monomial of degree $n+1$. If $a=u^{n+1}$ or $a=\left(u^{*}\right)^{n+1}$, then $a \in N$, hence $P_{0}(u)(a)=0$. Otherwise there are two monomials 
$b, c$ with $\operatorname{deg}(b)+\operatorname{deg}(c)=n+1$ such that either $a=b u u^{*} c$ or $a=b u^{*} u c$. If the first possibility takes place, then

$$
\begin{aligned}
P_{0}(u)(a) & =\left(1-u u^{*}\right) b u u^{*} c\left(1-u^{*} u\right) \\
& =\left(1-u u^{*}\right) b c\left(1-u^{*} u\right)-\left(1-u u^{*}\right) b\left(1-u u^{*}\right) c\left(1-u^{*} u\right) \\
& =P_{0}(u)(b c)-\left(1-u u^{*}\right) b P_{0}(u)(c)=0
\end{aligned}
$$

by the induction hypothesis. The second case is analogous.

Lemma 6.3. Let $M$ be a unital $J B^{*}$-algebra and $u$ a complete tripotent in $M$. Let $N$ be the closed unital Jordan *-subalgbebra of $M$ generated by $u$. Then there is a unital Jordan ${ }^{*}$-monomorphism $\psi: N \rightarrow B(H)$, where $H$ is a complex Hilbert space, such that $\psi(u)^{*} \psi(u)=1$.

Proof. By Lemma 6.2 we can assume that $N$ is a unital $\mathrm{JC}^{*}$-subalgebra of some $C^{*}$ algebra $A$ such that $u$ is a complete tripotent in $A$ as well. The desired conclusion follows now from Lemma 6.1.

6.3. Orders on tripotents. There is a natural partial order on tripotents which we recall below. We start by analyzing a coarser ordering (see the subsequent Proposition 6.5) which will be useful in the next section. We start by the following easy lemma.

Lemma 6.4. Let $M$ be a $J B^{*}$-triple, $e \in M$ a tripotent and $x \in M_{j}(e)$ for some $j \in\{0,1,2\}$. Then the following assertions hold.

(a) The operators $L(e, e)$ and $L(x, x)$ commute.

(b) The operator $L(x, x)$ commutes with the Peirce projections induced by e.

(c) If $x$ is moreover a tripotent, then the Peirce projections induced by $x$ commute with the Peirce projections induced by e.

Proof. (a) Let $j \in\{0,1,2\}$ be such that $x \in M_{j}(e)$. It means that $L(e, e) x=\frac{j}{2} x$. By the Jordan identity we deduce that given any $y \in M$ we have

$$
\begin{aligned}
L(e, e) L(x, x) y & =L(e, e)\{x, x, y\} \\
& =\{L(e, e) x, x, y\}-\{x, L(e, e) x, y\}+\{x, x, L(e, e) y\} \\
& =\left\{\frac{j}{2} x, x, y\right\}-\left\{x, \frac{j}{2} x, y\right\}+\{x, x, L(e, e) y\}=L(x, x) L(e, e) y .
\end{aligned}
$$

This completes the proof of (a). Assertions (b) and (c) follow from (a) using the formulae for Peirce projections.

Let us remark that statement $(c)$ was already established by G. Horn in 40, (1.10)]. The previous result and its proof are included here for completeness reasons.

A coarser ordering on the set of tripotents is considered in our next result.

Proposition 6.5. Let $M$ be a JB*-triple and e, $u$ be two tripotents in $M$. The following assertions are equivalent:

(1) $u \in M_{2}(e)$;

(2) $P_{2}(u) P_{2}(e)=P_{2}(e) P_{2}(u)=P_{2}(u), P_{1}(u) P_{1}(e)=P_{1}(e) P_{1}(u)$ and $P_{0}(u) P_{0}(e)=$ $P_{0}(e) P_{0}(u)=P_{0}(e)$

(3) $M_{2}(u) \subseteq M_{2}(e)$ and $M_{0}(e) \subseteq M_{0}(u)$;

(4) $M_{2}(u) \subseteq M_{2}(e)$. 
Proof. (1) $\Rightarrow(2)$ Assume that $u \in M_{2}(e)$. By Lemma 6.4 the Peirce projections induced by $u$ commute with the Peirce projections induced by $e$. Further, given $x \in M$ the Peirce rules (11) yield

$$
\left\{u, P_{1}(e) x, u\right\}=\left\{u, P_{0}(e) x, u\right\}=0,
$$

thus

$$
\{u, x, u\}=\left\{u, P_{2}(e) x, u\right\} .
$$

It follows that $Q(u)=Q(u) P_{2}(e)$, so

$$
P_{2}(u)=Q(u)^{2}=Q(u)^{2} P_{2}(e)=P_{2}(u) P_{2}(e) .
$$

Let $x \in M_{0}(e)$, another application of Peirce arithmetic yields

$$
\{u, u, x\} \in\left\{M_{2}(e), M_{2}(e), M_{0}(e)\right\}=\{0\},
$$

so $M_{0}(e) \subseteq M_{0}(u)$, and hence $P_{0}(u) P_{0}(e)=P_{0}(e)$.

The implications $(2) \Rightarrow(3) \Rightarrow(4) \Rightarrow(1)$ are obvious.

Proposition 6.6. Let $M$ be a JB*-triple, and let e, $u$ be two tripotents in $M$. The following assertions are equivalent:

(1) $u \in M_{2}(e)$ and $e \in M_{2}(u)$;

(2) $M_{2}(e)=M_{2}(u)$

(3) The Peirce decompositions induced by e and $u$ coincide (i.e., $M_{j}(e)=M_{j}(u)$ for all $j=0,1,2)$.

Proof. The implications $(3) \Rightarrow(2) \Rightarrow(1)$ are obvious.

$(1) \Rightarrow(3)$ Assume $u \in M_{2}(e)$ and $e \in M_{2}(u)$. It follows from Proposition 6.5 (the implication $(1) \Rightarrow(2)$ ) that $P_{2}(e)=P_{2}(u)$ and $P_{0}(e)=P_{0}(u)$. Hence also $P_{1}(u)=P_{1}(e)$.

Proposition 6.7. Let $M$ be a JB*-triple, and let e, $u$ be two tripotents in $M$. The following assertions are equivalent:

(1) $u \in M_{0}(e)$;

(2) $e \in M_{0}(u)$;

(3) $M_{2}(u) \subseteq M_{0}(e)$ and $M_{2}(e) \subseteq M_{0}(u)$;

(4) $P_{2}(u) P_{0}(e)=P_{0}(e) P_{2}(u)=P_{2}(u)$ and $P_{0}(u) P_{2}(e)=P_{2}(e) P_{0}(u)=P_{2}(e)$.

Proof. (1) $\Rightarrow(2)$ Assume $u \in M_{0}(e)$. Then $\{u, u, e\}=\{e, u, u\}=0$ by the Peirce arithmetics (note that $e \in M_{2}(e)$ and $u \in M_{0}(e)$ ). Hence $e \in M_{0}(u)$.

$(2) \Rightarrow(1)$ follows by symmetry.

$(1) \Rightarrow(4)$ Assume $u \in M_{0}(e)$. By the already proved implications we know that also $e \in M_{0}(u)$. It follows from Peirce arithmetic that $M_{2}(u) \subseteq M_{0}(e)$ and $M_{2}(e) \subseteq$ $M_{0}(u)$. Therefore $P_{2}(u)=P_{0}(e) P_{2}(u)$ and $P_{2}(e)=P_{0}(u) P_{2}(e)$. Since, by Peirce arithmetics, we also have $\left\{u, M_{2}(e), u\right\}=\left\{u, M_{1}(e), u\right\}=\{0\}$, and $P_{2}(u)=Q(u)^{2}$, we deduce that $P_{2}(u) P_{j}(e)=0$, for $j=1,2$. Therefore, $P_{2}(u)=P_{2}(u) P_{0}(e)$, and similarly $P_{2}(e)=P_{2}(e) P_{0}(u)$.

The implications $(4) \Rightarrow(3) \Rightarrow(2)$ are obvious.

Remark 6.8. Propositions 6.5 and 6.6 show that the Peirce subspace $M_{2}(e)$ determines the whole Peirce decomposition. This is not the case for $M_{0}(e)$ as there may exist complete tripotents with different Peirce decompositions. 
Tripotents $u, e \in M$ satisfying any of the equivalent conditions in Proposition 6.7 are called orthogonal ( $u \perp e$ in short). In particular, $e \pm u$ are again tripotents. It is actually known that given two tripotents $e, u \in M$, then $e \perp u$ if and only if $e \pm u$ is a tripotent (cf. [44, Lemma 3.6]).

We are now in position to recall the natural partial order on the set of tripotents. If $M$ is a $\mathrm{JB}^{*}$-triple and $e, u$ are two tripotents in $M$, we say that $u \leq e$ if $e-u$ is a tripotent orthogonal to $u$. This order is finer than the one derived from Proposition 6.5 as can be seen from the last of the characterizations in the following proposition (originally due to Y. Friedman and B. Russo [29, Corollary 1.7], compare also [19, Proposition 1.2.43], [17, Corollary 5.10.56]).

Proposition 6.9. (essentially [29, Corollary 1.7]) Let $M$ be a JB*-triple and $e, u \in$ $M$ two tripotents. Then the following assertions are equivalent:

- $u \leq e$;

- $u=P_{2}(u) e$;

- $u=\{u, e, u\}$;

- $u$ is a projection in the $J B^{*}$-algebra $M_{2}(e)$;

- $M_{2}(u)$ is a JB*-subalgebra of $M_{2}(e)$.

6.4. More on the Peirce-2 subspace. Our next result gathers some properties of the Peirce-2 subspace associated with a tripotent. Most of the statements are part of the folklore in the theory of $\mathrm{JB}^{*}$-triples, we include here the properties and basic references for completeness reasons.

Lemma 6.10. Let $M$ be a JB*-triple and let $e \in M$ be a tripotent. Consider $M_{2}(e)$ equipped with its structure of $J B^{*}$-algebra.

(a) Assume that $v \in M$ is a tripotent such that $e \leq v$. Then for any $a, b \in M$ we have

$$
\begin{aligned}
& P_{2}(e)\{a, b, v\}=\left\{P_{2}(e) a, P_{2}(e) b, e\right\}+\left\{P_{1}(e) a, P_{1}(e) b, e\right\}, \\
& P_{1}(e)\{a, b, v\}=\left\{P_{1}(e) a, P_{2}(e) b, e\right\}+\left\{P_{0}(e) a, P_{1}(e) b, e\right\} \quad+\left\{P_{2}(e) a, P_{1}(e) b, v-e\right\}+\left\{P_{1}(e) a, P_{0}(e) b, v-e\right\}, \\
& P_{0}(e)\{a, b, v\}=\left\{P_{0}(e) a, P_{0}(e) b, v-e\right\}+\left\{P_{1}(e) a, P_{1}(e) b, v-e\right\},
\end{aligned}
$$

in particular

$$
\begin{aligned}
& P_{2}(e)\{a, b, e\}=\left\{P_{2}(e) a, P_{2}(e) b, e\right\}+\left\{P_{1}(e) a, P_{1}(e) b, e\right\}, \\
& P_{1}(e)\{a, b, e\}=\left\{P_{1}(e) a, P_{2}(e) b, e\right\}+\left\{P_{0}(e) a, P_{1}(e) b, e\right\}, \\
& P_{0}(e)\{a, b, e\}=0 .
\end{aligned}
$$

(b) Assume $j \in\{1,2\}$ and $a, b \in M_{j}(e)$. Then

$$
\{a, b, e\} \in M_{2}(e) \text { and }\{a, b, e\}^{*}=\{b, a, e\} .
$$

(c) Assume $a, b \in M_{2}(e)$. Then $a \circ b^{*}=\{a, b, e\}$.

(d) If $a \in M_{2}(e) \cup M_{1}(e)$, then $\{a, a, e\}$ is a positive element of the JB*-algebra $M_{2}(e)$. Moreover, $\{a, a, e\}=0$ if and only if $a=0$.

Proof. Fix a tripotent $v \in M$ with $e \leq v$. Then $P_{2}(e) v=e, P_{1}(e) v=0$, and $P_{0}(e) v=v-e$. Hence the Peirce arithmetic implies that, given $x \in M_{j}(e)$ and 
$y \in M_{k}(e)$ for some $j, k \in\{0,1,2\}$, we have

$$
\{x, y, e\} \begin{cases}\in M_{2}(e) & \text { if } j=k=2 \text { or } j=k=1, \\ \in M_{1}(e) & \text { if } j=1, k=2 \text { or } j=0, k=1, \\ =0 & \text { otherwise, }\end{cases}
$$

and

$$
\{x, y, v-e\} \begin{cases}\in M_{0}(e) & \text { if } j=k=0 \text { or } j=k=1, \\ \in M_{1}(e) & \text { if } j=1, k=0 \text { or } j=2, k=1, \\ =0 & \text { otherwise. }\end{cases}
$$

Assertion $(a)$ now follows from (12) and (13). Further, the first statement of assertion $(b)$ follows from (12). Let us continue by proving the second statement from (b). We deduce from the Jordan identity, the definition of the involution in $M_{2}(e)$, and the fact that $\{b, a, e\} \in M_{2}(e)$, that

$$
\begin{aligned}
\{b, a, e\} & =L(b, a) e=L(b, a)\{e, e, e\} \\
& =\{L(b, a) e, e, e\}-\{e, L(a, b) e, e\}+\{e, e, L(b, a) e\} \\
& =2 L(e, e)\{b, a, e\}-(L(a, b) e)^{*}=2\{b, a, e\}-\{a, b, e\}^{*} .
\end{aligned}
$$

(c) The Peirce-2 subspace $M_{2}(e)$ is a $\mathrm{JB}^{*}$-algebra, and hence it is a $\mathrm{JB}^{*}$-triple with respect to the triple product given by $\{a, b, c\}_{1}=\left(a \circ b^{*}\right) \circ c+\left(c \circ b^{*}\right) \circ$ $a-(a \circ c) \circ b^{*}$. It is also a $\mathrm{JB}^{*}$-triple with the triple product inherited from $M$. Since the identity mapping from $\left(M_{2}(e),\{., ., .\}_{1}\right)$ onto $\left(M_{2}(e),\{., .,\}.\right)$ is a surjective isometry, it follows from Kaup's Riemann mapping theorem (see [52, Proposition 5.5] or [19, Theorem 3.1.7]) that $\{a, b, c\}_{1}=\{a, b, c\}$, for all $a, b, c \in$ $M_{2}(e)$. Consequently, $\{a, b, e\}=\{a, b, e\}_{1}=\left(a \circ b^{*}\right) \circ e+\left(e \circ b^{*}\right) \circ a-(a \circ e) \circ b^{*}=a \circ b^{*}$, because $e$ is the unit of $M_{2}(e)$.

(d) If $a \in M_{2}(e)$, then $\{a, a, e\}=a \circ a^{*}$ by $(c)$, hence the assertion follows from Lemma 3.1(ii). The case $a \in M_{1}(e)$ is covered by [29, Lemma 1.5(b)], and both cases $\left(a \in M_{1}(e)\right.$ and $\left.a \in M_{2}(e)\right)$ are fully studied in 63] (see also [16, Proposition $4.2 .32]$ ), where a simple proof based on the axioms of $\mathrm{JB}^{*}$-triples can be found.

When we combine the previous result with the properties of the functionals in the dual space of a $\mathrm{JB}^{*}$-triple we get additional properties. We recall that a functional $\varphi$ in the dual space of a $\mathrm{JB}^{*}$-algebra $M$ is called faithful if $\varphi(a)=0$ for $a \geq 0$ implies $a=0$.

Lemma 6.11. Let $M$ be a $J B^{*}$-triple and let $e \in M$ be a tripotent. Consider $M_{2}(e)$ equipped with its structure of unital $J B^{*}$-algebra. Let $\varphi \in M^{*}$. Then the following assertions hold:

(a) $\left\|\varphi \circ\left(P_{2}(e)+P_{0}(e)\right)\right\|=\left\|\varphi \circ P_{2}(e)\right\|+\left\|\varphi \circ P_{0}(e)\right\|$.

Moreover, if $\|\varphi\|=\varphi(e)$, then the following assertions are valid, too:

(b) $\varphi=\varphi \circ P_{2}(e)$;

(c) $\left.\varphi\right|_{M_{2}(e)}$ is a positive linear functional on the $J B^{*}$-algebra $M_{2}(e)$;

(d) The mapping

$$
(x, y) \mapsto \varphi(\{x, y, e\}), \quad x, y \in M,
$$

is a positive semidefinite sesquilinear form on $M$, and if $z \in M$ is a norm-one element satisfying $\varphi(z)=\|\varphi\|$ then $\varphi(\{x, y, e\})=\varphi(\{x, y, z\})$ for all $x, y \in M$; 
(e) The formula

$$
\|x\|_{e, \varphi}=\sqrt{\varphi(\{x, x, e\})}, \quad x \in M
$$

defines a pre-Hilbert seminorm on $M$ which is zero on $M_{0}(e)$.

If moreover $\left.\varphi\right|_{M_{2}(e)}$ is faithful, then the kernel of $\|\cdot\|_{e, \varphi}$ is exactly $M_{0}(e)$.

Proof. Assertions $(a)$ and $(b)$ are proved in 29, Lemma 1.3(b) and Proposition $1(a)$ ], compare also [17, Lemma 5.7.11, Fact 5.10.53].

(c) Since $\|\varphi\|=\varphi(e)$ we also have $\left\|\varphi \circ P_{2}(e)\right\|=\|\varphi\|=\left\|\left.\varphi\right|_{M_{2}(e)}\right\|=\left.\varphi\right|_{M_{2}(e)}(e)$. Therefore $\left.\varphi\right|_{M_{2}(e)}$ is a positive functional on the $\mathrm{JB}^{*}$-algebra $M_{2}(e)$ (cf. 36, Lemma 1.2.2] or [17, Lemma 5.10.2]).

$(d)$ and $(e)$ are consequences of $(c),(b)$ and Lemma $6.10(a)$ and $(d)$. They are also explicitly proved in [6, Proposition 1.2] and [24, Lemma 4.1]. See also [17, Proposition 5.10.60] for the $\mathrm{JBW}^{*}$-case.

\section{STRONG* TOPOLOGY AND WEAKLY COMPACT SETS}

In the previous section we collected many results on projections and tripotents. However, it may happen that there are no nontrivial projections or tripotents. For example, the $C^{*}$-algebra $\mathcal{C}_{0}(\mathbb{R})$ contains no nonzero projection or tripotent and in the unital $C^{*}$-algebra $\mathcal{C}([0,1])$ the only projections are 0 and 1 and the only nonzero tripotents are the unitary ones (which coincide with the continuous functions with values in the unit circle). The situation is different in the dual case - in a von Neumann algebra projections form a complete lattice and their linear span is normdense, and, as we previously commented, any $\mathrm{JBW}^{*}$-triple provides a rich supply of tripotents (cf. [19, Theorem 3.2.3] or [16, Theorem 4.2.34]).

If $M$ is a $\mathrm{JBW}^{*}$-triple and $u \in M$ is a tripotent, then the Peirce projections are weak*-to-weak* continuous and the Peirce subspaces are weak*-closed. This follows from the separate weak*-to-weak* continuity of the triple product and the explicit formulae for the Peirce projections displayed in page 15. In particular, $M_{2}(u)$ is a $\mathrm{JBW}^{*}$-algebra.

7.1. Strong* topology on $\mathbf{J B W}^{*}$-triples. Assume that $M$ is a $\mathrm{JBW}^{*}$-triple and $\varphi \in M_{*} \backslash\{0\}$. By [29, Proposition 2] (see also [17, Proposition 5.10.57]) there is a unique tripotent $s(\varphi) \in M$, called the support tripotent of $\varphi$, such that

- $\varphi=\varphi \circ P_{2}(s(\varphi))$,

- $\left.\varphi\right|_{M_{2}(s(\varphi))}$ is a faithful positive functional on the $\mathrm{JBW}^{*}$-algebra $M_{2}(s(\varphi))$.

Furthermore, $\|\varphi\|=\varphi(s(\varphi))$. According to the notation from Lemma 6.11, we set $\|\cdot\|_{\varphi}=\|\cdot\|_{s(\varphi), \varphi}$.

Note that if $M$ is a $\mathrm{JBW}^{*}$-algebra (or even a von Neumann algebra) and $\varphi \in M_{*}$ is a positive functional, then its support tripotent $s(\varphi)$ is even a projection because in such a case $\varphi$ attains its norm at a positive element. Observe that in the latter case the seminorm $\|\cdot\|_{\varphi}$ writes in the form

$$
\|x\|_{\varphi}=\sqrt{\varphi\{x, x, s(\varphi)\}}=\sqrt{\varphi\{x, x, 1\}}=\sqrt{\varphi\left(x^{*} \circ x\right)} .
$$

Introduced in [7, the strong* topology on $M$ is the locally convex topology generated by the seminorms $\|\cdot\|_{\varphi}$ where $\varphi$ runs in the set $M_{*} \backslash\{0\}$. It should be noted that in the original definition (see [7, Definition 3.1]) only norm-one functionals are considered, but both definitions obviously give the same notion. Since each 
$\|\cdot\|_{\varphi}$ is a preHilbertian seminorm, it follows from the Cauchy-Schwarz inequality (cf.6.11(d)) and the properties of the support tripotent that, with $x_{2}=P_{2}(s(\varphi)) x$,

$$
\begin{aligned}
|\varphi(x)| & =\left|\varphi\left(x_{2}\right)\right|=\left|\varphi\left(x_{2} \circ_{s(\varphi)} s(\varphi)\right)\right|=\left|\varphi\left(\left\{x_{2}, s(\varphi), s(\varphi)\right\}\right)\right| \\
& \stackrel{6.10}{=}\left|\varphi\left(P_{2}(s(\varphi))\{x, s(\varphi), s(\varphi)\}\right)\right|=|\varphi\{x, s(\varphi), s(\varphi)\}| \\
& \leq\|x\|_{\varphi}\|s(\varphi)\|_{\varphi}=\sqrt{\|\varphi\|}\|x\|_{\varphi}, \quad x \in M, \varphi \in M_{*} \backslash\{0\} .
\end{aligned}
$$

Consequently, the strong* topology is stronger than the weak* topology. by

Given $\varphi_{1}, \ldots, \varphi_{n} \in M_{*}$, we shall write $\|\cdot\|_{\varphi_{1}, \ldots, \varphi_{n}}$ for the seminorm on $M$ defined

$$
\|x\|_{\varphi_{1}, \ldots, \varphi_{n}}^{2}:=\sum_{k=1}^{n}\|x\|_{\varphi_{k}}^{2}(x \in M) .
$$

The following lemma summarizes some known properties of the strong* topology.

Lemma 7.1. Let $M$ be a $J B W^{*}$-triple.

(a) If $M$ is even a JBW*-algebra, then the strong* topology on $M$ coincides with the algebra strong* topology, i.e., with the locally convex topology generated by seminorms

$$
x \mapsto \sqrt{\varphi\left(x^{*} \circ x\right)}, \quad \varphi \in M_{*}, \varphi \geq 0 ;
$$

(b) If $N$ is a weak ${ }^{*}$ closed subtriple of $M$, then the strong* topology of $N$ coincides with the restriction to $N$ of the strong* topology of $M$;

(c) If $M$ is even a von Neumann algebra (embedded to some $B(H)$ ), the strong* topology coincides on bounded sets with the locally convex topology generated by the seminorms

$$
x \mapsto\|x \xi\|+\left\|x^{*} \xi\right\|, \quad \xi \in H ;
$$

(d) A linear functional $\varphi: M \rightarrow \mathbb{C}$ is strong* continuous if and only if it is weak ${ }^{*}$ continuous. Furthermore a linear mapping between JBW*-triples is strong ${ }^{*}$-tostrong* continuous if and only if it is weak ${ }^{*}-$ to-weak $k^{*}$ continuous. In particular, the Peirce projections associated with a tripotent are strong ${ }^{*}$-to-strong* continuous;

(e) If $s(\varphi)$ is complete, then $\|x\|_{\varphi}$ is a norm on $M$.

Proof. Assertion $(a)$ is proved in [68, Proposition 3], while $(b)$ is established in [14, COROLLARY].

Let us justify assertion $(c)$. In [77, Definition II.2.3] the name $\sigma$-strong* operator topology is used for the algebra strong* topology in $B(H)$. By [77, Lemma II.2.5(iii)] this topology coincides on bounded sets with the topology generated by the given seminorms. Hence we can conclude by applying $(a)$ and $(b)$.

Statement $(d)$ is proved in [65, Corollary 9] and [68, Corollary 3] and the comments before [65, Theorem 9].

(e) If $s(\varphi)$ is a complete tripotent, then $M_{0}(s(\varphi))=\{0\}$, thus the statement follows from Lemma 6.11 $(e)$.

The description of the strong* topology is closely related to $\sigma$-finite projections and tripotents. Recall that a projection $p$ in a $\mathrm{JBW}^{*}$-algebra is called $\sigma$-finite if any family of pairwise orthogonal smaller nonzero projections is at most countable. If the unit of a $\mathrm{JBW}^{*}$-algebra is $\sigma$-finite, the respective algebra is called $\sigma$-finite. The classical definitions in von Neumann algebras are exactly the same. Let us note 
that some authors also employ the term countably decomposable to refer to $\sigma$-finite projections in a von Neumann algebra (cf. [72, Definition 2.1.8] or [49, Definition 5.5.14]).

Similarly, a tripotent $u$ in a $\mathrm{JBW}^{*}$-triple is $\sigma$-finite if any family of pairwise orthogonal nonzero smaller tripotents is at most countable. A $\mathrm{JBW}^{*}$-triple is itself called $\sigma$-finite if it admits a $\sigma$-finite complete tripotent (cf. [24, §3]). The next result gathers some basic facts on $\sigma$-finite tripotents.

Let us recall a couple of notions. A subspace $I$ of a $\mathrm{JB}^{*}$-triple $E$ is an inner ideal if $\{I, E, I\} \subseteq I$. Every inner ideal of $E$ is a subtriple.

Given a norm-one element $a$ in a $\mathrm{JBW}^{*}$-triple $M$, there exists a smallest tripotent $e \in M$ satisfying that $a$ is a positive element in the $\mathrm{JBW}^{*}$-algebra $M_{2}(e)$, this tripotent is called the range tripotent of $a$, and it will be denoted by $r(a)$ (see, for example, [23, comments before Lemma 3.1]). For a non-zero element $b \in M$, the range tripotent of $b, r(b)$, is defined as the range tripotent of $\frac{b}{\|b\|}$ and we set $r(0)=0$. It follows from the same reference that if $M$ is a $\mathrm{JBW}^{*}$-algebra and $x$ is a non-zero positive element, then $r(x)$ is a projection and it coincides with the range projection in [36, Lemma 4.2.6].

Lemma 7.2. (a) Let $u$ be a tripotent in a $J B W^{*}$-triple $M$. Then $u$ is $\sigma$-finite if and only if $u=s(\varphi)$ for some norm-one functional $\varphi \in M_{*}$;

(b) Let $M$ be a JB $W^{*}$-algebra and $p \in M$ a projection. Then $p$ is a $\sigma$-finite projection if and only if it is a $\sigma$-finite tripotent;

(c) Let $M$ be a JB $W^{*}$-algebra and $p \in M$ a projection. Then $p$ is $\sigma$-finite if and only if $p=s(\varphi)$ for some normal state (i.e., a positive norm-one functional) $\varphi \in M_{*}$;

(d) Let $M$ be a JBW*-algebra and $e \in M$ a $\sigma$-finite tripotent. Then there is a $\sigma$-finite projection $p \in M$ such that $e \in M_{2}(p)$.

Proof. Assertion (a) is proved in 24, Theorem 3.2]. Statement (b) follows from the fact that any projection is also a tripotent, and from the property that a tripotent $u$ is smaller than or equal to a projection $p$ if and only if $u$ is a projection and $u \leq p$ (cf. Proposition 6.9).

Statement $(c)$ is a consequence of $(a)$.

(d) Let us consider the sets

$$
\begin{aligned}
S & =\left\{x \in M: \exists p \in M \text { a } \sigma \text {-finite projection such that } x \in M_{2}(p)\right\}, \\
M_{\sigma} & =\left\{x \in M: \exists u \in M \text { a } \sigma \text {-finite tripotent such that } x \in M_{2}(u)\right\} .
\end{aligned}
$$

Clearly $S \subseteq M_{\sigma}$. By [11, page 667 and Theorems 4.1 and 5.1] we have $M_{\sigma}=\{x \in$ $M: r(x)$ is $\sigma$-finite $\}$ is (a 1-norming $\Sigma$-subspace and) a norm-closed inner ideal of $M=\left(M_{*}\right)^{*}$ (see the quoted paper for definitions). Since $M$ is a $\mathrm{JBW}^{*}$-algebra, we get $M_{\sigma} \circ M_{\sigma}=\left\{M_{\sigma}, 1, M_{\sigma}\right\} \subseteq M_{\sigma}$, and hence $M_{\sigma}$ is a Jordan subalgebra of $M$.

It can be seen easily that a tripotent $u \in M$ is $\sigma$-finite if and only if $u^{*}$ is, therefore $M_{\sigma}^{*} \subseteq M_{\sigma}$, and hence $M_{\sigma}^{*}=M_{\sigma}$ is a norm-closed JB*-subalgebra of $M$, which is also hereditary in the Jordan terminology, that is, if $0 \leq a \leq b$ in $M$ with $b \in M_{\sigma}$, then $a \in M_{\sigma}$.

Let $a=h+i k$ be an element in $M_{\sigma}$, where $h, k \in\left(M_{\sigma}\right)_{s a}$. The elements $h^{2}, k^{2}$ are positive and belong to $M_{\sigma}$, thus $h^{2}+k^{2} \in M_{\sigma}$. Therefore, the range tripotent $p=r\left(h^{2}+k^{2}\right)$ is a $\sigma$-finite projection in $M$. Clearly, $h^{2}, k^{2} \leq h^{2}+k^{2} \in M_{2}(p) \subseteq M_{\sigma}$, 
and consequently, $h, k \in M_{2}(p)$, which implies that $p \circ h=h$ and $p \circ k=k$. Therefore $\{p, p, a\}=p \circ a=a$ as desired.

Lemme 2 in Appendice 6 in 43 offers a sufficient condition to guarantee the metrizability of the strong* topology on the bounded subsets of a JBW-algebra. We shall next adapt the result to $\mathrm{JBW}^{*}$-algebras.

Lemma 7.3. Let $M$ be a $\sigma$-finite $J B W^{*}$-algebra.

(a) $M$ admits a faithful normal state;

(b) Let $\varphi$ be a faithful normal state on $M$. Then the topology induced by the norm

$$
x \mapsto \sqrt{\varphi\left(x^{*} \circ x\right)}=\|x\|_{\varphi}, \quad x \in M,
$$

coincides with the strong* topology on bounded sets.

Proof. (a) Since 1 is a $\sigma$-finite projection, by Lemmal7.2(c) there is a positive normone functional $\varphi \in M_{*}$ (i.e., a normal state) such that $s(\varphi)=1$. It follows that $\varphi$ is faithful.

(b) We know that $M_{s a}$ is a JBW-algebra and a real $\mathrm{JBW}^{*}$-subtriple of $M$, and that $\left.\varphi\right|_{M_{s a}}$ is a faithful normal state on $M_{s a}$. [43, Appendice 6, Lemme 2] implies that the strong* topology on the closed unit ball of $M_{s a}$ is metrized by the seminorm $\|x\|_{\varphi}^{2}=\varphi(x \circ x)=\varphi\left(x^{2}\right), x \in M_{s a}$. Let $\left(a_{\lambda}\right)_{\lambda}$ be a net in $B_{M}$, and $a \in B_{M}$ such that $\left\|a_{\lambda}-a\right\|_{\varphi} \rightarrow 0$. If we write $a_{\lambda}=h_{\lambda}+i k_{\lambda}$ and $a=h+i k$ with $h_{\lambda}, k_{\lambda}, h, k \in B_{M_{s a}}$, then we get the inequalities

$$
\varphi\left(\left(h_{\lambda}-h\right)^{2}\right), \varphi\left(\left(k_{\lambda}-k\right)^{2}\right) \leq \varphi\left(\left(h_{\lambda}-h\right)^{2}+\left(k_{\lambda}-k\right)^{2}\right)=\left\|a_{\lambda}-a\right\|_{\varphi}^{2} .
$$

Therefore, $\left(h_{\lambda}\right)_{\lambda} \rightarrow h$ and $\left(k_{\lambda}\right)_{\lambda} \rightarrow k$ in the strong* topology of $M_{s a}$, and by [14, COROLLARY] they also converge to the same limits with respect to the strong* topology of $M$, and consequently, $\left(a_{\lambda}\right)_{\lambda} \rightarrow a$ in the strong* topology of $M$.

The previous lemma says, in particular, that the strong* topology is metrizable on bounded sets of a $\sigma$-finite $\mathrm{JBW}^{*}$-algebra. The analogous statement for von Neumann algebras is proved already in [77, Proposition III.5.3]. The analogy for $\mathrm{JBW}^{*}$-triples fails, as we will explain below (see Example 9.1).

We continue now with a lemma characterizing strong* convergence of bounded positive nets.

Lemma 7.4. Let $\left(x_{\nu}\right)$ be a bounded net of positive elements in a JBW*-algebra $M$. Then the following assertions are equivalent.

(1) $x_{\nu} \stackrel{\text { strong* }}{\longrightarrow} 0$;

(2) $\varphi\left(x_{\nu}\right) \rightarrow 0$ for each positive $\varphi \in M_{*}$;

(3) $x_{\nu} \stackrel{\text { weak }}{\longrightarrow} 0$.

Proof. The implication $(1) \Rightarrow(3)$ follows from the fact that the strong* topology is stronger than the weak* one, and $(3) \Rightarrow(2)$ is trivial.

$(2) \Rightarrow(1)$ : By Lemma 7.1(a) the net $\left(x_{\nu}\right)$ strong* $^{*}$ converges to zero if and only if for any positive $\varphi \in M_{*}$ we have $\varphi\left(x_{\nu}^{2}\right) \rightarrow 0$. Now the double inequality $0 \leq x_{\nu}^{2} \leq$ $\left\|x_{\nu}\right\| x_{\nu}$ proves the implication $(2) \Rightarrow(1)$. 
7.2. Order on seminorms generating the strong* topology. To describe the strong* topology it is not necessary (in some cases) to use all the defining seminorms. This is witnessed by Lemma 7.1)(a) and, on bounded sets, by Lemma 7.3. In this section we investigate this feature in detail. A key result is the following proposition relating the order on seminorms with the order from Proposition 6.5.

Proposition 7.5. Let $M$ be a $J B W^{*}$-triple and $\varphi, \psi \in M_{*} \backslash\{0\}$.

(i) Assume $s(\psi) \in M_{2}(s(\varphi))$ (or, equivalently, $\left.M_{2}(s(\psi)) \subseteq M_{2}(s(\varphi))\right)$. Then the seminorm $\|\cdot\|_{\psi}$ is weaker than the seminorm $\|\cdot\|_{\varphi}$ on bounded sets.

(ii) Assume $M_{2}(s(\psi)) \varsubsetneqq M_{2}(s(\varphi))$. Then on $B_{M}$, the seminorm $\|\cdot\|_{\psi}$ is strictly weaker than the seminorm $\|\cdot\|_{\varphi}$.

To prove this proposition we will need some lemmata. The first lemma characterizes convergence of sequences in a fixed seminorm. Note that the same characterization applies to nets, but since we are comparing seminorms, sequences are enough.

Lemma 7.6. Let $M$ be a JBW*-triple and $\varphi \in M_{*} \backslash\{0\}$. Let $e=s(\varphi)$ and let $\left(a_{n}\right)$ be a bounded sequence in $M$. Then the following assertions are equivalent:

(1) $\left\|a_{n}\right\|_{\varphi} \rightarrow 0$;

(2) $\left\{P_{2}(e)\left(a_{n}\right), P_{2}(e)\left(a_{n}\right), e\right\} \stackrel{\text { strong }}{\longrightarrow} 0$ and $\left\{P_{1}(e)\left(a_{n}\right), P_{1}(e)\left(a_{n}\right), e\right\} \stackrel{\text { strong }}{\longrightarrow} 0$;

(3) $\left\{P_{2}(e)\left(a_{n}\right), P_{2}(e)\left(a_{n}\right), e\right\} \stackrel{\text { weak }^{*}}{\longrightarrow} 0$ and $\left\{P_{1}(e)\left(a_{n}\right), P_{1}(e)\left(a_{n}\right), e\right\} \stackrel{\text { weak }}{\longrightarrow} 0$;

(4) $\left\{P_{2}(e)\left(a_{n}\right), P_{2}(e)\left(a_{n}\right), e\right\}+\left\{P_{1}(e)\left(a_{n}\right), P_{1}(e)\left(a_{n}\right), e\right\} \stackrel{\text { weak }}{\longrightarrow} 0$.

Proof. First notice, that it does not matter whether the convergence is considered in the $\mathrm{JBW}^{*}$-triple $M$ or in the $\mathrm{JBW}^{*}$-algebra $M_{2}(e)$ (cf. [14, COROLLARY]). The strong* case follows from Lemma $7.1(a),(b)$, the weak* case is obvious.

$(1) \Rightarrow(2)$ Assume that $\left\|a_{n}\right\|_{\varphi} \rightarrow 0$, i.e., $\varphi\left(\left\{a_{n}, a_{n}, e\right\}\right) \rightarrow 0$. Since $\varphi=\varphi \circ P_{2}(e)$, by Lemma $6.10(a)$ we have

$$
\varphi\left(\left\{a_{n}, a_{n}, e\right\}\right)=\varphi\left(\left\{P_{2}(e)\left(a_{n}\right), P_{2}(e)\left(a_{n}\right), e\right\}+\left\{P_{1}(e)\left(a_{n}\right), P_{1}(e)\left(a_{n}\right), e\right\}\right) .
$$

We actually know that the elements

$$
w_{n}=\left\{P_{2}(e)\left(a_{n}\right), P_{2}(e)\left(a_{n}\right), e\right\} \text { and } z_{n}=\left\{P_{1}(e)\left(a_{n}\right), P_{1}(e)\left(a_{n}\right), e\right\}
$$

are positive in the $\mathrm{JBW}^{*}$-algebra $M_{2}(e)$ by Lemma $6.10(d)$. Note that $0 \leq w_{n}^{2} \leq$ $\left\|w_{n}\right\| \cdot w_{n}$. Since the sequence $\left(w_{n}\right)$ is bounded, we deduce $\varphi\left(w_{n}^{2}\right) \rightarrow 0$, hence $w_{n} \stackrel{\text { strong* }}{\longrightarrow} 0$ by Lemma $7.3(b)$. Similarly we get $z_{n} \stackrel{\text { strong* }}{\longrightarrow} 0$.

$(2) \Rightarrow(3)$ This is clear, as the weak* topology is weaker than the strong* one.

$(3) \Rightarrow(4)$ This follows by the linearity of the weak* topology.

$(4) \Rightarrow(1)$ This follows from the fact that

$$
\left\|a_{n}\right\|_{\varphi}^{2}=\varphi\left(\left\{P_{2}(e)\left(a_{n}\right), P_{2}(e)\left(a_{n}\right), e\right\}+\left\{P_{1}(e)\left(a_{n}\right), P_{1}(e)\left(a_{n}\right), e\right\}\right) .
$$

The following lemma, together with the preceding one, provides a proof of assertion $(i)$ of Proposition 7.5 . 
Lemma 7.7. Let $M$ be a $J B W^{*}$-triple and $p, u \in M$ tripotents such that $u \in M_{2}(p)$. Then for any bounded sequence $\left(a_{n}\right)$ in $M$ we have

$$
\begin{aligned}
\left\{P_{2}(p)\left(a_{n}\right)\right. & \left., P_{2}(p)\left(a_{n}\right), p\right\}+\left\{P_{1}(p)\left(a_{n}\right), P_{1}(p)\left(a_{n}\right), p\right\} \stackrel{\text { weak }^{*}}{\longrightarrow} 0 \\
& \Longrightarrow\left\{P_{2}(u)\left(a_{n}\right), P_{2}(u)\left(a_{n}\right), u\right\}+\left\{P_{1}(u)\left(a_{n}\right), P_{1}(u)\left(a_{n}\right), u\right\} \stackrel{\text { weak }}{\longrightarrow} 0 .
\end{aligned}
$$

Proof. Let us start by noticing that we can identify $M$ with a $\mathrm{JB}^{*}$-subtriple of a unital $\mathrm{JB}^{*}$-algebra $B$ in such a way that $p$ is a projection in $B$. This is proved in the first paragraph of the proof of [15, Proposition 2.4] (using [30, Corollary 1] and [15, Lemma 2.3]). (Note that $B$ can be assumed to be a $\mathrm{JBW}^{*}$-algebra - just pass to $B^{* *}$.)

Let us consider the bounded linear mapping $G: B \rightarrow B_{2}(u)$ defined by

$$
G(x)=P_{2}(u)(x \circ u), \quad(x \in B) .
$$

Further, observe that for any $x \in B$ we have

$$
\begin{aligned}
\{x, x, u\}+\left\{x^{*}, x^{*}, u\right\}= & \left(x \circ x^{*}\right) \circ u+\left(x^{*} \circ u\right) \circ x-(x \circ u) \circ x^{*} \\
& +\left(x \circ x^{*}\right) \circ u+(u \circ x) \circ x^{*}-\left(x^{*} \circ u\right) \circ x \\
= & 2\left(x \circ x^{*}\right) \circ u,
\end{aligned}
$$

thus

$$
\begin{aligned}
P_{2}(u)\left(\{x, x, u\}+\left\{x^{*}, x^{*}, u\right\}\right) & =2 P_{2}(u)\left(\left(x \circ x^{*}\right) \circ u\right) \\
& =2 G\left(x \circ x^{*}\right)=2 G(\{x, x, 1\}) .
\end{aligned}
$$

Further, given any $x \in B$, we have

$$
\begin{aligned}
P_{2}(p)(\{x, x, 1\} \circ u) & =P_{2}(p)\{\{x, x, 1\}, 1, u\} \\
& =\left\{P_{2}(p)\{x, x, 1\}, P_{2}(p)(1), u\right\}+\left\{P_{1}(p)\{x, x, 1\}, P_{1}(p)(1), u\right\} \\
& =\left\{P_{2}(p)\{x, x, 1\}, p, u\right\}=\left\{P_{2}(p)\{x, x, 1\}, 1, u\right\} \\
& =\left(P_{2}(p)\{x, x, 1\}\right) \circ u=\left(P_{2}(p)\{x, x, p\}\right) \circ u .
\end{aligned}
$$

Indeed, the first equality is obvious, the second one follows from Peirce arithmetic as $u \in M_{2}(p) \subseteq B_{2}(p)$. In the third equality we use the facts that $P_{2}(p)(1)=p$ and $P_{1}(p)(1)=0$. The fourth equality follows by Peirce arithmetic using the fact that $1-p \in B_{0}(p)$, and thus $1-p \perp u$. The fifth equality is obvious and the sixth one follows from Lemma $6.10(a)$.

Thus, for each $x \in B$ we have

$$
\begin{aligned}
G(\{x, x, 1\}) & =P_{2}(u)(\{x, x, 1\} \circ u)^{\left.\frac{6.5}{=} 2\right)} P_{2}(u) P_{2}(p)(\{x, x, 1\} \circ u) \\
& =P_{2}(u)\left(\left(P_{2}(p)\{x, x, p\}\right) \circ u\right)=G\left(P_{2}(p)\{x, x, p\}\right),
\end{aligned}
$$

so

$$
P_{2}(u)\left(\{x, x, u\}+\left\{x^{*}, x^{*}, u\right\}\right)=2 G\left(P_{2}(p)\{x, x, p\}\right) .
$$

Let us check what happens in $M$. If $x \in M$, then

$$
\begin{aligned}
G(x) & =P_{2}(u)(x \circ u)=P_{2}(u) P_{2}(p)\{x, 1, u\} \\
& =P_{2}(u)\left(\left\{P_{2}(p)(x), P_{2}(p)(1), u\right\}+\left\{P_{1}(p)(x), P_{1}(p)(1), u\right\}\right) \\
& =P_{2}(u)\left\{P_{2}(p) x, p, u\right\} .
\end{aligned}
$$

It follows that $G$ maps $M$ into $P_{2}(u)(M)=M_{2}(u)$ and, moreover, $G$ restricted to $M$ is weak*-to-weak* continuous. 
So, assume $\left(a_{n}\right)$ is a bounded sequence in $M$ such that

$$
\left\{P_{2}(p)\left(a_{n}\right), P_{2}(p)\left(a_{n}\right), p\right\}+\left\{P_{1}(p)\left(a_{n}\right), P_{1}(p)\left(a_{n}\right), p\right\} \stackrel{\text { weak }^{*}}{\longrightarrow} 0
$$

equivalently,

$$
P_{2}(p)\left\{a_{n}, a_{n}, p\right\} \stackrel{\text { weak }^{*}}{\longrightarrow} 0 .
$$

Since this sequence lives in $M$, using weak*-to-weak* continuity of $G$ we get

$$
G\left(P_{2}(p)\left\{a_{n}, a_{n}, p\right\}\right) \stackrel{\text { weak }^{*}}{\longrightarrow} 0,
$$

and this sequence is contained in $M_{2}(u)$. Note that by the above calculation and Lemma 6.10(a) we have

$$
\begin{aligned}
G\left(P_{2}(p)\left\{a_{n}, a_{n}, p\right\}\right)= & \frac{1}{2} P_{2}(u)\left(\left\{a_{n}, a_{n}, u\right\}+\left\{a_{n}^{*}, a_{n}^{*}, u\right\}\right) \\
= & \frac{1}{2}\left(\left\{P_{2}(u)\left(a_{n}\right), P_{2}(u)\left(a_{n}\right), u\right\}+\left\{P_{1}(u)\left(a_{n}\right), P_{1}(u)\left(a_{n}\right), u\right\}\right. \\
& \left.+\left\{P_{2}(u)\left(a_{n}^{*}\right), P_{2}(u)\left(a_{n}^{*}\right), u\right\}+\left\{P_{1}(u)\left(a_{n}^{*}\right), P_{1}(u)\left(a_{n}^{*}\right), u\right\}\right) .
\end{aligned}
$$

Moreover, all the four summands in the right hand side are positive elements in the JB*-algebra $B_{2}(u)$ by Lemma $6.10(d)$, hence their sum is positive as well. Moreover, the sum belongs to $M_{2}(u)$ and the first two summands as well, and thus

$$
\left\{P_{2}(u)\left(a_{n}^{*}\right), P_{2}(u)\left(a_{n}^{*}\right), u\right\}+\left\{P_{1}(u)\left(a_{n}^{*}\right), P_{1}(u)\left(a_{n}^{*}\right), u\right\} \in M_{2}(u),
$$

too. Since

$$
\begin{aligned}
0 \leq\{ & \left.P_{2}(u)\left(a_{n}\right), P_{2}(u)\left(a_{n}\right), u\right\}+\left\{P_{1}(u)\left(a_{n}\right), P_{1}(u)\left(a_{n}\right), u\right\} \\
\leq\{ & \left.P_{2}(u)\left(a_{n}\right), P_{2}(u)\left(a_{n}\right), u\right\}+\left\{P_{1}(u)\left(a_{n}\right), P_{1}(u)\left(a_{n}\right), u\right\} \\
& \quad+\left\{P_{2}(u)\left(a_{n}^{*}\right), P_{2}(u)\left(a_{n}^{*}\right), u\right\}+\left\{P_{1}(u)\left(a_{n}^{*}\right), P_{1}(u)\left(a_{n}^{*}\right), u\right\},
\end{aligned}
$$

the equivalence (2) $\Leftrightarrow(3)$ in Lemma $7.4(2)$ shows that

$$
\left\{P_{2}(u)\left(a_{n}\right), P_{2}(u)\left(a_{n}\right), u\right\}+\left\{P_{1}(u)\left(a_{n}\right), P_{1}(u)\left(a_{n}\right), u\right\} \stackrel{\text { weak }^{*}}{\longrightarrow} 0,
$$

which completes the proof.

The following lemma together with Lemma 7.6 provides the proof for assertion (ii) in Proposition 7.5 .

Lemma 7.8. Let $M$ be a $J B W^{*}$-triple and $e, u \in M$ two tripotents such that $M_{2}(u) \varsubsetneqq M_{2}(e)$. Then there is a bounded sequence $\left(a_{n}\right)$ in $M$ such that

$$
\left\{P_{2}(u)\left(a_{n}\right), P_{2}(u)\left(a_{n}\right), u\right\} \stackrel{\text { strong }}{\longrightarrow} 0 \text { and }\left\{P_{1}(u)\left(a_{n}\right), P_{1}(u)\left(a_{n}\right), u\right\} \stackrel{\text { strong }^{*}}{\longrightarrow} 0
$$

but

$$
\left\{P_{2}(e)\left(a_{n}\right), P_{2}(e)\left(a_{n}\right), e\right\}+\left\{P_{1}(e)\left(a_{n}\right), P_{1}(e)\left(a_{n}\right), e\right\} \stackrel{\text { strong }^{*}}{\nrightarrow} 0 .
$$

Proof. If $M_{0}(u) \cap M_{2}(e)$ contains a nonzero element $a$, then

$$
\left\{P_{2}(u)(a), P_{2}(u)(a), u\right\}=\left\{P_{1}(u)(a), P_{1}(u)(a), u\right\}=0
$$

but

$$
\left\{P_{2}(e)(a), P_{2}(e)(a), e\right\}+\left\{P_{1}(e)(a), P_{1}(e)(a), e\right\}=\{a, a, e\} \neq 0
$$

by Lemma $6.10(d)$. It follows that the constant sequence $a_{n}=a$ works. 
Next assume that $M_{0}(u) \cap M_{2}(e)$ is trivial, hence $u$ is a complete tripotent in $M_{2}(e)$. Since $M_{2}(e)$ is a JBW*-algebra, by Lemma 7.1 $(b)$ it is enough to consider the case in which $M=M_{2}(e)$. We shall therefore assume that $M$ is a $\mathrm{JBW}^{*}$-algebra, $e=1$ and $u \in M$ is a complete non-unitary tripotent.

Let $N$ denote the unital $\mathrm{JB}^{*}$-subalgebra of $M$ generated by $u$. By Lemma 6.3 we can assume without loss of generality that $N$ is a $J B^{*}$-subalgebra of $B(H)$ for a suitable complex Hilbert space $H$ and, moreover, $u^{*} u=1$ in $B(H)$. Since $u$ is not unitary, necessarily $u u^{*} \neq 1$.

Set $q=u u^{*}$. Then $q$ is a projection in $B(H)$. Moreover, $q \in N$, as

$$
q=u u^{*}=u u^{*}+u^{*} u-1=2 u \circ u^{*}-1 .
$$

Let us define a sequence in $N$ by $x_{0}=1-q$ and $x_{n}=x_{n-1} \circ u^{*}$ for $n \in \mathbb{N}$. We claim that

$$
x_{n}=2^{-n}(1-q)\left(u^{*}\right)^{n}, \quad n \in \mathbb{N} \cup\{0\} .
$$

Indeed, for $n=0$ the equality holds. Assume that $n \in \mathbb{N}$ and the equality holds for $n-1$. Then

$$
\begin{aligned}
x_{n} & =x_{n-1} \circ u^{*}=\frac{1}{2}\left(x_{n-1} u^{*}+u^{*} x_{n-1}\right)=2^{-n}\left((1-q)\left(u^{*}\right)^{n}+u^{*}(1-q)\left(u^{*}\right)^{n-1}\right) \\
& =2^{-n}(1-q)\left(u^{*}\right)^{n},
\end{aligned}
$$

as obviously $u^{*}(1-q)=0$.

Set $a_{n}=2^{n} x_{n}=(1-q)\left(u^{*}\right)^{n}$. Then $\left(a_{n}\right)$ is a bounded sequence in $N$, and hence in $M$. Further,

$$
\begin{aligned}
\left\{a_{n}, a_{n}, 1\right\} & =a_{n} \circ a_{n}^{*}=\frac{1}{2}\left(a_{n} a_{n}^{*}+a_{n}^{*} a_{n}\right) \\
& =\frac{1}{2}\left(u^{n}(1-q)\left(u^{*}\right)^{n}+(1-q)\left(u^{*}\right)^{n} u^{n}(1-q)\right) \\
& =\frac{1}{2}\left(u^{n}(1-q)\left(u^{*}\right)^{n}+(1-q)\right)
\end{aligned}
$$

as $u^{*} u=1$. Therefore,

$$
\left\{a_{n}, a_{n}, 1\right\} \geq 1-q,
$$

and it then follows from Lemma 7.4 that $\left\{a_{n}, a_{n}, 1\right\}$ does not converge to zero in the strong* topology.

Further, observe that $q=P_{2}(u)(1)$, hence $q \in M_{2}(u)$ and $1-q \in M_{1}(u)$. We claim that $x_{n} \in M_{1}(u)$ for every $n \in \mathbb{N} \cup\{0\}$. The case, $n=0$ is clear. The Peirce arithmetic yields by the induction hypothesis that

$$
\begin{aligned}
x_{n} & =x_{n-1} \circ u^{*}=\left\{x_{n-1}, u, 1\right\}=\left\{x_{n-1}, u, q\right\}+\left\{x_{n-1}, u, 1-q\right\} \\
& =\left\{x_{n-1}, u, q\right\} \in M_{1}(u),
\end{aligned}
$$

where we used that $\left\{x_{n-1}, u, 1-q\right\} \in M_{0}(u)=\{0\}$. So $a_{n} \in M_{1}(u)$ for each $n \in \mathbb{N} \cup\{0\}$ as well. It follows by Lemma 6.10 (a) that

$$
\begin{aligned}
P_{2}(u)\left\{a_{n}, a_{n}, u\right\} & =\left\{a_{n}, a_{n}, u\right\}=\frac{1}{2}\left(a_{n} a_{n}^{*} u+u a_{n}^{*} a_{n}\right) \\
& =\frac{1}{2}\left((1-q)\left(u^{*}\right)^{n} u^{n}(1-q) u+u u^{n}(1-q)(1-q)\left(u^{*}\right)^{n}\right) \\
& =\frac{1}{2} u^{n+1}(1-q)\left(u^{*}\right)^{n}
\end{aligned}
$$


as $(1-q) u=0$. We shall show that the sequence $\frac{1}{2} u^{n+1}(1-q)\left(u^{*}\right)^{n}$ strong* converges to zero.

To this end note that $u^{n}$ is a partial isometry for each $n \in \mathbb{N}$ and, moreover, $\left(u^{n}\right)^{*} u^{n}=1$. Thus its final projection $q_{n}=u^{n}\left(u^{n}\right)^{*}$ belongs to $N$. Let

$$
Y=\overline{\bigcup_{n \in \mathbb{N}} \operatorname{ker}\left(\left(u^{n}\right)^{*}\right)}
$$

Then $Y$ is a closed subspace of $H$, and $\lim _{n}\left(u^{*}\right)^{n}(\xi)=0$ for each $\xi \in Y$.

Furthermore,

$$
Y^{\perp}=\bigcap_{n \in \mathbb{N}}\left(\operatorname{ker}\left(\left(u^{n}\right)^{*}\right)\right)^{\perp}=\bigcap_{n \in \mathbb{N}}\left(\operatorname{ker} q_{n}\right)^{\perp}=\bigcap_{n \in \mathbb{N}} q_{n}(H) .
$$

Thus for any $\xi \in Y^{\perp}$ and $n \in \mathbb{N}$ we have $\xi=q_{n}(\xi)$ and so

$$
\begin{aligned}
(1-q)\left(u^{*}\right)^{n}(\xi) & =(1-q)\left(u^{*}\right)^{n} q_{n+1}(\xi)=(1-q)\left(u^{*}\right)^{n} u^{n+1}\left(u^{*}\right)^{n+1}(\xi) \\
& =(1-q) u\left(u^{*}\right)^{n+1}(\xi)=(1-q) q\left(u^{*}\right)^{n}(\xi)=0 .
\end{aligned}
$$

It follows that the sequence $(1-q)\left(u^{*}\right)^{n}$ SOT converges to zero, hence clearly $\left(\frac{1}{2} u^{n+1}(1-q)\left(u^{*}\right)^{n}\right)$ strong* converges to zero. This completes the proof.

7.3. Weakly compact sets in the predual of a $\mathbf{J B W}^{*}$-triple. There is a close connection of the strong* topology, the generating seminorms and the weakly compact subsets of the predual. It is witnessed, for example, by the following proposition which is proved in [69, Theorem D.21], see also [17, Theorem 5.10.138].

Proposition 7.9. Let $M$ be a JBW*-triple. The strong* topology on bounded subsets of $M$ coincides with the Mackey topology (i.e., with the topology of uniform convergence on weakly compact subsets of $\left.M_{*}\right)$.

We shall analyze the relationship in more detail in the following lemma.

Lemma 7.10. Assume that $M$ is a $J B W^{*}$-triple, $e \in M$ is a tripotent, and $\varphi \in M_{*}$ satisfies $\|\varphi\|=\varphi(e)$. Define the mapping $\Phi=\Phi_{e, \varphi}: M \rightarrow M_{*}$ by

$$
\Phi(a)(x)=\varphi(\{x, a, e\}), \quad x \in M, a \in M .
$$

(a) $\Phi$ is a conjugate linear mapping of $M$ into $M_{*}$ which is moreover weak ${ }^{*}$-to-weak continuous and $\|\Phi\| \leq\|\varphi\|$;

(b) Set $K=K(e, \varphi)=\Phi\left(B_{M}\right) \subseteq\|\varphi\| B_{M_{*}}$. Then $K$ is an absolutely convex weakly compact subset of $M_{*}$;

(c) Let $a \in M$ be arbitrary. Then

$$
\sup \{|\psi(a)| ; \psi \in K\}=\|\Phi(a)\| ;
$$

(d) For each $x \in B_{M}$ we have

$$
\|x\|_{e, \varphi}^{2} \leq\|\Phi(x)\| \leq \sqrt{\|\varphi\|} \cdot\|x\|_{e, \varphi} .
$$

In particular, the topologies induced by the seminorms $\|\cdot\|_{e, \varphi}$ and $\|\Phi(\cdot)\|$ coincide on $B_{M}$.

Proof. (a) The mapping

$$
(x, y) \mapsto \varphi(\{x, y, e\}), \quad x, y \in M,
$$


is a separately weak* ${ }^{*}$-continuous sesquilinear form on $M$ (cf. Lemma 6.11). Indeed, the separate weak* continuity follows from the assumption $\varphi \in M_{*}$ together with the separate weak*-to-weak* continuity of the Jordan product.

It follows that for each $a \in M$ its image $\Phi(a)$ is a weak* continuous linear functional on $M$, hence $\Phi(a) \in M_{*}$. Further, $\Phi$ is clearly conjugate linear. The estimate of the norm is immediate from the inequality $\|\{x, y, z\}\| \leq\|x\|\|y\|\|z\|$ $(x, y, z \in M)$ [30, Corollary 3], [16. Corollary 4.1.114]. Finally, $\Phi$ is weak*-to-weak continuous because for each $x \in\left(M_{*}\right)^{*}=M$ the mapping

$$
a \mapsto \Phi(a)(x)=\varphi(\{x, a, e\})
$$

is weak* continuous on $M$.

(b) This follows from $(a)$ as $B_{M}$ is weak* compact and absolutely convex.

(c) Let us compute:

$$
\begin{aligned}
\sup \{|\psi(a)| ; \psi \in K\} & =\sup \left\{|\Phi(x)(a)| ; x \in B_{M}\right\}=\sup \left\{|\varphi(\{a, x, e\})| ; x \in B_{M}\right\} \\
& =\sup \left\{|\overline{\varphi(\{x, a, e\})}| ; x \in B_{M}\right\}=\sup \left\{|\overline{\Phi(a)(x)}| ; x \in B_{M}\right\} \\
& =\|\Phi(a)\|
\end{aligned}
$$

where we used that the sesquilinear form from the proof of $(a)$ is hermitian (because it is even positive semidefinite by Lemma 6.11 $(d))$.

(d) Fix any $x \in B_{M}$. Then

$$
\|x\|_{e, \varphi}^{2}=\varphi(\{x, x, e\})=\Phi(x)(x) \leq\|\Phi(x)\|,
$$

which proves the first inequality. Further, for any $y \in B_{M}$, by the Cauchy-Schwarz inequality, we have

$$
|\Phi(x)(y)|=|\varphi(\{y, x, e\})| \leq\|x\|_{e, \varphi} \cdot\|y\|_{e, \varphi} \leq\|x\|_{e, \varphi} \cdot \sqrt{\|\Phi(y)\|} \leq \sqrt{\|\varphi\|} \cdot\|x\|_{e, \varphi} .
$$

This proves the second inequality. The 'in particular part'then follows immediately.

If $\varphi \in M_{*} \backslash\{0\}$, we set

$$
\Phi_{\varphi}=\Phi_{s(\varphi), \varphi}, K(\varphi)=K(s(\varphi), \varphi),\|\cdot\|_{\varphi}^{K}=\left\|\Phi_{\varphi}(\cdot)\right\|,
$$

where we use the notation from the previous lemma.

We can next state a characterization of relatively weakly compact subsets in the predual of a $\mathrm{JBW}^{*}$-triple.

Proposition 7.11. Let $M$ be a $J B W^{*}$-triple. Let $A \subseteq M_{*} \backslash\{0\}$ be such that the topology on $M$ generated by the family $\left\{\|\cdot\|_{\varphi}: \varphi \in A\right\}$ coincides on bounded sets with the strong* topology. Then the following assertions are satisfied.

(a) Let $L \subseteq M_{*}$ be a weakly compact subset and $\varepsilon>0$. Then there are $\varphi_{1}, \ldots, \varphi_{k} \in$ $A$ and $n \in \mathbb{N}$ such that

$$
L \subseteq n \cdot \operatorname{conv}\left(K\left(\varphi_{1}\right) \cup \cdots \cup K\left(\varphi_{k}\right)\right)+\varepsilon B_{M_{*}} ;
$$

(b) Assume moreover that the family $\left\{M_{2}(s(\varphi)) ; \varphi \in A\right\}$ is up-directed by inclusion. Then for any weakly compact set $L \subseteq M_{*}$ and any $\varepsilon>0$ there are $\varphi \in A$ and $n \in \mathbb{N}$ such that $L \subseteq n K(\varphi)+\varepsilon B_{M_{*}}$.

In particular, the assumption is satisfied if

$$
\forall \psi \in M_{*} \backslash\{0\}, \exists \varphi \in A: s(\psi) \in M_{2}(s(\varphi)) .
$$


Proof. (a) We may assume that $0<\varepsilon \leq 1$. We will use the following notation. For a bounded set $D \subseteq M_{*}$ denote by $q_{D}$ the seminorm on $M$ defined by

$$
q_{D}(x)=\sup \{|\varphi(x)| ; \varphi \in D\} .
$$

Then $q_{L}$ is a Mackey continuous seminorm on $M$, so $\left.q_{L}\right|_{B_{M}}$ is strong* continuous by Proposition 7.9. The assumption together with Lemma 7.10 $(d)$ yields the existence of $\varphi_{1}, \ldots, \varphi_{k} \in A$ and a natural number $m$ such that for $\delta=\frac{1}{m}>0$ we have

$$
\left\{x \in B_{M} ;\|x\|_{\varphi_{j}}^{K} \leq \delta \text { for } j=1, \ldots, k\right\} \subseteq\left\{x \in B_{M} ; q_{L}(x) \leq \varepsilon\right\},
$$

hence

$$
\left\{x \in B_{M} ;\|x\|_{\varphi_{j}}^{K} \leq \delta \text { for } j=1, \ldots, k\right\}_{\circ} \supset\left\{x \in B_{M} ; q_{L}(x) \leq \varepsilon\right\}_{\circ} .
$$

Clearly

$$
\left\{x \in B_{M} ; q_{L}(x) \leq \varepsilon\right\}_{\circ} \supset \frac{1}{\varepsilon} L .
$$

Further, by Lemma $\mathbf{7 . 1 0}(c)$ we have $\|\cdot\|_{\varphi}^{K}=q_{K(\varphi)}$ for any $\varphi \in A$, hence

$$
\begin{aligned}
\left\{x \in B_{M} ;\right. & \left.\|x\|_{\varphi_{j}}^{K} \leq \delta \text { for } j=1, \ldots, k\right\}_{\circ}=\left(B_{M} \cap \bigcap_{j \leq k}\left\{x \in M ; q_{K\left(\varphi_{j}\right)}(x) \leq \delta\right\}\right) \\
& \left.=\left(B_{M} \cap \bigcap_{j \leq k}\left(\frac{1}{\delta} K\left(\varphi_{j}\right)\right)^{\circ}\right)_{\circ}=\left(B_{M_{*}} \cup \frac{1}{\delta} \bigcup_{j \leq k} K\left(\varphi_{j}\right)\right)^{\circ}\right)_{\circ} \\
& \subseteq \frac{1}{\delta} \operatorname{conv}\left(K\left(\varphi_{1}\right) \cup \cdots \cup K\left(\varphi_{k}\right)\right)+B_{M_{*}}
\end{aligned}
$$

It follows that

$$
\begin{aligned}
L & \subseteq \bar{\varepsilon} \frac{\bar{c} \operatorname{conv}\left(K\left(\varphi_{1}\right) \cup \cdots \cup K\left(\varphi_{k}\right)\right)+\varepsilon B_{M_{*}}}{\delta} \\
& \subseteq m \cdot \operatorname{conv}\left(K\left(\varphi_{1}\right) \cup \cdots \cup K\left(\varphi_{k}\right)\right)+2 \varepsilon B_{M_{*}},
\end{aligned}
$$

which completes the proof.

(b) We proceed in the same way as in the proof of $(a)$. We find $\varphi_{1}, \ldots, \varphi_{k}$ and $\delta$. The assumption then yields $\varphi \in A$ such that $M_{2}(s(\varphi))$ contains $s\left(\varphi_{1}\right), \ldots, s\left(\varphi_{k}\right)$. By Proposition $7.5(i)$ and Lemma $7.10(d)$ we get some $\eta>0$ such that

$$
\begin{aligned}
\left\{x \in B_{M} ;\|x\|_{\varphi}^{K} \leq \eta\right\} & \subseteq\left\{x \in B_{M} ;\|x\|_{\varphi_{j}}^{K} \leq \delta \text { for } j=1, \ldots, k\right\} \\
& \subseteq\left\{x \in B_{M} ; q_{L}(x) \leq \varepsilon\right\} .
\end{aligned}
$$

The arguments in the second part of the proof of $(a)$ complete the proof here.

The 'in particular' statement concerning the family $\left\{\|\cdot\|_{\varphi}: \varphi \in A\right\}$ follows from Proposition $7.5(i)$.

\section{Proof of the main Result}

In this section we provide a proof of Theorem 4.1 We begin with a technical lemma. 
Lemma 8.1. Let $M$ be a $J B W^{*}$-algebra and let $\left(p_{n}\right)$ be an increasing sequence of projections in $M$ with supremum $p$. Then for any bounded sequence $\left(a_{k}\right)$ in $M$ we have

$$
\text { strong }{ }^{*}-\lim _{k} P_{2}(p)\left\{a_{k}, a_{k}, p\right\}=0 \Leftrightarrow \forall n \in \mathbb{N}, \text { strong }^{*}-\lim _{k} P_{2}\left(p_{n}\right)\left\{a_{k}, a_{k}, p_{n}\right\}=0 .
$$

Proof. $(\Rightarrow)$ Fix $n \in \mathbb{N}$. By Lemma 6.10( $a$ ) we have

$$
P_{2}\left(p_{n}\right)\left\{a_{k}, a_{k}, p_{n}\right\}=P_{2}\left(p_{n}\right)\left\{a_{k}, a_{k}, p\right\}=P_{2}\left(p_{n}\right) P_{2}(p)\left\{a_{k}, a_{k}, p\right\} .
$$

Thus we can conclude by the strong*-to-strong* continuity of $P_{2}\left(p_{n}\right)$.

$(\Leftarrow)$ Arguing by contradiction, we assume that $\left(a_{k}\right)$ is a bounded sequence in $M$ such that

$$
\forall n \in \mathbb{N}: \text { strong* }{ }^{*} \lim _{k} P_{2}\left(p_{n}\right)\left\{a_{k}, a_{k}, p_{n}\right\}=0
$$

strong*

but $P_{2}(p)\left\{a_{k}, a_{k}, p\right\} \stackrel{\text { sto }}{\longrightarrow} 0$. We may assume, without loss of generality that $\left(a_{k}\right) \subseteq B_{M}$. Since

$$
P_{2}(p)\left\{a_{k}, a_{k}, p\right\}=\left\{P_{2}(p)\left(a_{k}\right), P_{2}(p)\left(a_{k}\right), p\right\}+\left\{P_{1}(p)\left(a_{k}\right), P_{1}(p)\left(a_{k}\right), p\right\}
$$

is a positive element of $M$ (by Lemma 6.10 $(a),(d)$ ), there is, due to Lemma 7.4 a positive norm-one functional $\varphi \in M_{*}$ (i.e., a normal state on $M$ ) such that $\varphi\left(P_{2}(p)\left\{a_{k}, a_{k}, p\right\}\right) \not \rightarrow 0$. Up to passing to a subsequence we may assume that there is some $c>0$ such that

$$
\varphi\left(P_{2}(p)\left\{a_{k}, a_{k}, p\right\}\right)>c, \text { for all } k \in \mathbb{N} .
$$

By [10, Lemma 3.2] there is some $m \in \mathbb{N}$ with $\left\|P_{2}(p)^{*} \varphi-P_{2}\left(p_{m}\right)^{*} \varphi\right\|<\frac{c}{2}$. Then

$$
\begin{aligned}
\varphi\left(P_{2}\left(p_{m}\right)\left\{a_{k}, a_{k}, p_{m}\right\}\right) & =\varphi\left(P_{2}\left(p_{m}\right)\left\{a_{k}, a_{k}, p\right\}\right) \\
& >c+\varphi\left(P_{2}\left(p_{m}\right)\left\{a_{k}, a_{k}, p\right\}\right)-\varphi\left(P_{2}(p)\left\{a_{k}, a_{k}, p\right\}\right) \\
& =c+P_{2}\left(p_{m}\right)^{*} \varphi\left\{a_{k}, a_{k}, p\right\}-P_{2}(p)^{*} \varphi\left\{a_{k}, a_{k}, p\right\} \\
& \geq c-\left\|P_{2}\left(p_{m}\right)^{*} \varphi-P_{2}(p)^{*} \varphi\right\|>\frac{c}{2}
\end{aligned}
$$

for all $k \in \mathbb{N}$. Thus, Lemma 7.4 implies that $\left(P_{2}\left(p_{m}\right)\left\{a_{k}, a_{k}, p_{m}\right\}\right)_{k} \stackrel{\text { strong* }^{*}}{\longrightarrow} 0$ leading to a contradiction.

Lemma 8.2. Let $M$ be a $\sigma$-finite $J B W^{*}$-algebra and let $\left(\varphi_{n}\right)$ be a sequence of nonzero positive functionals in $M_{*}$ such that their support projections $s\left(\varphi_{n}\right)$ form an increasing sequence with supremum 1 . Then the strong* topology on bounded subsets of $M$ coincides with the topology generated by the seminorms $\|\cdot\|_{\varphi_{n}}, n \in \mathbb{N}$.

Proof. Since $M$ is $\sigma$-finite, there exists a normal state $\varphi \in M_{*}$ with $s(\varphi)=1$ and, moreover, the norm $\|\cdot\|_{\varphi}$ generates the strong* topology on bounded sets of $M$ (cf. Lemma (7.3). Hence we can conclude using Lemmata 7.6 and 8.1 .

Lemma 8.3. Let $M$ be a $J B W^{*}$-algebra and $A \subseteq M_{*}$ a bounded set. Then there is a countable set $B \subseteq A$ such that $\omega\left(B^{\prime}\right)=\omega(A)$ for any $B^{\prime} \subseteq B$ infinite.

Proof. For any $\sigma$-finite projection $p \in M$, the Peirce- 2 subspace $M_{2}(p)$ is a $\sigma$-finite $\mathrm{JBW}^{*}$-algebra, hence by Lemma 7.3 we can fix a faithful normal state $\omega_{p}$ on $M_{2}(p)$. Let us set $\varphi_{p}=\omega_{p} \circ P_{2}(p)$. Then $\varphi_{p}$ is a normal positive functional on $M$ such that $\left\|\varphi_{p}\right\|=\varphi_{p}(p)=1$ and $\left.\varphi_{p}\right|_{M_{2}(p)}$ is faithful. 
Let $\Phi_{p}=\Phi_{p, \varphi_{p}}$ using the notation from Lemma 7.10. Let $K_{p}=K\left(p, \varphi_{p}\right)=$ $\Phi_{p}\left(B_{M}\right)$. Then $K_{p}$ is a weakly compact set in $M_{*}$ (by Lemma 7.10(b)).

Let $A \subseteq M_{*}$ be a bounded set such that $c=\omega(A)>0$. Let us construct, by induction, two sequences $\left(\gamma_{n}\right) \subseteq A$ and $\left(p_{n}\right) \subseteq M$ such that

(i) $\left\|\gamma_{1}\right\|>c-1$

(ii) $p_{n}$ is a $\sigma$-finite projection such that $\gamma_{n}=\gamma_{n} \circ P_{2}\left(p_{n}\right)$;

(iii) $p_{n} \geq p_{k}$ for $k<n$;

(iv) $\operatorname{dist}\left(\gamma_{n+1}, n \operatorname{conv}\left(K_{p_{1}} \cup \cdots \cup K_{p_{n}}\right)\right)>c-\frac{1}{n+1}$.

This construction can be done by just applying the definition of $\omega(A)$. Indeed, the existence of $\gamma_{1} \in A$ satisfying (i) is obvious. Assume that $n \in \mathbb{N}$ and we have already constructed $\gamma_{j}$ for $1 \leq j \leq n$ and $p_{j}$ for $1 \leq j<n$.

By [10, Lemma 3.6] there is a $\sigma$-finite projection $r \in M$ such that $\gamma_{n}=\gamma_{n} \circ P_{2}(r)$. By [10. Lemma 3.5], there is a $\sigma$-finite projection $p_{n} \geq r$ satisfying (iii). Clearly $p_{n}$ satisfies (ii) as well. Finally, find $\gamma_{n+1} \in A$ satisfying (iv) by the definition of $\omega(A)$.

Set $B=\left\{\gamma_{n} ; n \in \mathbb{N}\right\}$. Then $B$ is a countable subset of $A$ and we claim that $\omega\left(B^{\prime}\right)=c$ for each infinite subset $B^{\prime} \subseteq B$.

Let $p=\sup _{n} p_{n}$. Then $p$ is $\sigma$-finite (see, e.g., [24, Theorem 3.4] or [10, Lemma 3.5]) and $B \subseteq P_{2}(p)^{*} M_{*}$. Since $P_{2}(p)$ is a norm-one projection, an application of Lemma 2.1 shows that $\omega(B)=\omega_{M_{2}(p)}(B)$. We continue by working in the JBW*algebra $M_{2}(p)$.

Lemma 8.2 implies that the strong* topology on $B_{M_{2}(p)}$ is generated by the sequence of seminorms $\|\cdot\|_{\left.\varphi_{p_{n}}\right|_{M_{2}(p)}}$. Let $L \subseteq\left(M_{2}(p)\right)_{*}=P_{2}(p)^{*} M_{*}$ a weakly compact set and $\varepsilon>0$. By Proposition 7.11(b) there are $m, n \in \mathbb{N}$ such that

$$
L \subseteq n \Phi_{p_{m}}\left(B_{M_{2}(p)}\right)+\varepsilon B_{M_{2}(p)_{*}} \subseteq n\left(K_{p_{m}} \cap P_{2}(p)^{*} M_{*}\right)+\varepsilon B_{M_{2}(p)_{*}} .
$$

It follows that

$$
\widehat{\mathrm{d}}(B, L) \geq \widehat{\mathrm{d}}\left(B, n K_{p_{m}}\right)-\varepsilon \geq \widehat{\mathrm{d}}\left(B, k K_{p_{m}}\right)-\varepsilon \geq c-\frac{1}{k+1}-\varepsilon
$$

for each $k \geq \max \{n, m\}$. Hence $\widehat{\mathrm{d}}(B, L) \geq c-\varepsilon$. Since $L$ is an arbitrary weakly compact set, we get $\omega(B) \geq c-\varepsilon$, and by the arbitrariness of $\varepsilon>0$ we have $\omega(B) \geq c$.

The same procedure applies to each infinite subset $B^{\prime} \subseteq B$, so the proof is completed.

Proof of Theorem 4.1. If $M$ is a JBW*-algebra, the result follows from Lemma 8.3 27. Corollary 4.3], and Proposition 5.1(b). The general case of a $\mathrm{JBW}^{*}$-triple follows from the $\mathrm{JBW}^{*}$-algebra case applying Lemma 2.1 and Proposition 3.2 .

\section{Preduals of JBW*-triples Which are strongly WCG}

Strongly WCG spaces (see the end of Section 5 for definitions) are a nice class of Banach spaces in which the computation of the De Blasi measure of weak noncompactness is easy. As explained in the end of Section 5 they include the spaces $L^{1}(\mu)$ for a $\sigma$-finite measure $\mu$ or, more generally, preduals of $\sigma$-finite von Neumann algebras and preduals of $\sigma$-finite $\mathrm{JBW}^{*}$-algebras. In the present section we characterize $\mathrm{JBW}^{*}$-triples whose preduals are strongly WCG.

Let us explain why it is not clear. By [73, Theorem 2.1] a Banach space $X$ is strongly WCG if and only if the Mackey topology on $X^{*}$ is metrizable on bounded 
sets. Therefore, it follows from Proposition 7.9 that the predual $M_{*}$ of a $\mathrm{JBW}^{*}$ triple $M$ is strongly WCG if and only if the strong* topology on $B_{M}$ is metrizable. For $\sigma$-finite $\mathrm{JBW}^{*}$-algebras this is the case by Lemma 7.3. However, for $\sigma$-finite $\mathrm{JBW}^{*}$ triples it need not be the case as witnessed by the following example.

Example 9.1. Let $\Gamma$ be an uncountable set and $C \subseteq \Gamma$ be an infinite countable set. Then $M=B\left(\ell^{2}(\Gamma), \ell^{2}(C)\right)$ is a $\sigma$-finite $J B W^{*}$-triple whose strong* topology is not metrizable on the closed unit ball of $M$.

Proof. A family of pairwise orthogonal partial isometries of $M=B\left(\ell^{2}(\Gamma), \ell^{2}(C)\right)$ has pairwise orthogonal final projections and is therefore countable as $\ell^{2}(C)$ is separable. Hence $M$ is $\sigma$-finite. $M$ is 1 -complemented in $B\left(\ell^{2}(\Gamma)\right)$ by a weak*to-weak* continuous projection, thus by [72, §1.15] and [14, COROLLARY], the strong* topology on bounded sets of $M$ is given by the seminorms $a \mapsto\left\|a\left(e_{\gamma}\right)\right\|+$ $\left\|a^{*}\left(e_{\gamma}\right)\right\|(a \in M)$, where $\gamma$ is a fixed element in $\Gamma$ and $\left\{e_{\gamma}: \gamma \in \Gamma\right\}$ is the canonical orthonormal basis of $\ell^{2}(\Gamma)$.

If the strong* topology of $B_{M}$ were metrizable, it would be first countable, hence there would exist a countable set $D \subseteq \Gamma$ such that the seminorms $a \mapsto$ $\left\|a\left(e_{\gamma}\right)\right\|+\left\|a^{*}\left(e_{\gamma}\right)\right\|, \gamma \in D$, generate this topology. We may assume without loss of generality that $D \supset C$. Let $C=\left(c_{n}\right)_{n \in \mathbb{N}}$. Let $\left\{\gamma_{n}: n \in \mathbb{N}\right\}$ be a set of pairwise distinct elements in $\Gamma \backslash D$. Define the sequence of operators $a_{k} \in M$ by

$$
a_{k}\left(e_{\gamma}\right)= \begin{cases}e_{c_{n+k}} & \text { if } \gamma=\gamma_{n} \\ 0 & \text { otherwise }\end{cases}
$$

Then

$$
a_{k}^{*}\left(e_{\gamma}\right)= \begin{cases}e_{\gamma_{n-k}} & \text { if } \gamma=c_{n} \text { and } n>k \\ 0 & \text { otherwise. }\end{cases}
$$

In this case $\left\|a_{k}\left(e_{\gamma_{n}}\right)\right\|=1$, so $a_{k}$ do not converge strong* to zero. However $a_{k}^{*} \rightarrow 0$ in SOT and $a_{k}\left(e_{\gamma}\right)=0$ for $\gamma \in D$, so $\left\|a_{k}\left(e_{\gamma}\right)\right\|+\left\|a_{k}^{*}\left(e_{\gamma}\right)\right\| \rightarrow 0$ for $\gamma \in D$, leading to a contradiction.

On the other hand, $\sigma$-finiteness of a $\mathrm{JBW}^{*}$-triple is a necessary condition for its predual to be strongly WCG. Indeed, any strongly WCG space is clearly WCG and the predual of a $\mathrm{JBW}^{*}$-triple is $\mathrm{WCG}$ if and only if the triple is $\sigma$-finite by [11, Theorem 1.1].

In order to find a sufficient and necessary condition we get back to the structure results of $\mathrm{JBW}^{*}$-triples due to G. Horn and E. Neher presented in (1) in page 7 (see [41, (1.7)], [42, (1.20)]). Every $\mathrm{JBW}^{*}$-triple $M$ decomposes (uniquely) as an (orthogonal) $\ell_{\infty}$-sum of the form $M=\left(\bigoplus_{j \in \mathcal{J}} A_{j} \bar{\otimes} C_{j}\right)_{\ell_{\infty}} \oplus_{\ell_{\infty}} H(W, \alpha) \oplus_{\ell_{\infty}} p V$, where each $A_{j}$ is a commutative von Neumann algebra, each $C_{j}$ is a Cartan factor, $W$ and $V$ are continuous von Neumann algebras, $p$ is a projection in $V, \alpha$ is a linear involution on $W$ commuting with ${ }^{*}$, that is, a linear *-antiautomorphism of period 2 on $W$, and $H(W, \alpha)=\{x \in W: \alpha(x)=x\}$. Clearly, $H(W, \alpha)$ is a $\mathrm{JBW}^{*}$-subalgebra of $W$ when the latter is equipped whit its natural structure of $\mathrm{JBW}^{*}$-algebra.

A Cartan factor of type 1 is a $\mathrm{JBW}^{*}$-triple $C_{1}$ which coincides with the space $B(H, K)$ of all bounded linear operators between two complex Hilbert spaces $H$ and $K$. We can always assume that $K$ is a closed subspace of $H$. Therefore, denoting 
by $p$ the orthogonal projection of $H$ onto $K$, we have $C_{1}=B(H, K)=p B(H)$. Suppose $A$ is a commutative von Neumann algebra. Now taking $\widehat{p}=1 \otimes p \in A \bar{\otimes} C_{1}$, we deduce that $A \bar{\otimes} C_{1}=\widehat{p}(A \bar{\otimes} B(H))$ is a right ideal of the von Neumann algebra $A \bar{\otimes} B(H)$.

Cartan factors of types 2 and 3 are the subtriples of $B(H)$ defined by $C_{2}=\{x \in$ $\left.B(H): x=-j x^{*} j\right\}$ and $C_{3}=\left\{x \in B(H): x=j x^{*} j\right\}$, respectively, where $j$ is a conjugation (i.e., a conjugate-linear isometry of period 2) on $H$. By a little abuse of notation, each $x \in B(H)$ can be identified with a "matrix" $\left(x_{\gamma \delta}\right)_{\gamma, \delta \in \Gamma}$. It is easy to check that the representing matrix of $j x^{*} j$ is the transpose of the representing matrix of $x$. Hence, $C_{2}$ consists of operators with antisymmetric representing matrix and $C_{3}$ of operators with symmetric ones.

The properties around Peirce decomposition show that, if a $\mathrm{JBW}^{*}$-triple $M$ admits a unitary element $u$, then $M=M_{2}(u)$ is a $\mathrm{JBW}^{*}$-algebra with product $\circ_{u}$ and involution $*_{u}$ (cf. page [16). It is shown in the proof of [38, Proposition 2] that every Cartan factor of type 2 with $\operatorname{dim}(H)$ even, or infinite, and every Cartan factor of type 3 contains a unitary element. The same result actually proves that Cartan factors of type 2 with $\operatorname{dim}(H)$ even, or infinite, and all Cartan factors of type 3 are $\mathrm{JBW}^{*}$-algebras. Consequently, if $C$ is a Cartan factor of type 2 with $\operatorname{dim}(H)$ even, or infinite, or a Cartan factor of type 3 , and $A$ is a commutative von Neumann algebra, then $A \bar{\otimes} C$ is a $\mathrm{JBW}^{*}$-algebra.

A Cartan factor of type 4 (also called a spin factor) is a complex Hilbert space (with inner product $\langle.,$.$\rangle ) equipped with a conjugation x \mapsto \bar{x}$, triple product

$$
\{x, y, z\}=\langle x, y\rangle z+\langle z, y\rangle x-\langle x, \bar{z}\rangle \bar{y}
$$

and norm given by $\|x\|^{2}=\langle x, x\rangle+\sqrt{\langle x, x\rangle^{2}-|\langle x, \bar{x}\rangle|^{2}}$. Let $u$ be an element in a spin factor $C_{4}$ satisfying $u=\bar{u}$ and $\|u\|=\langle u, u\rangle=1$. It is not hard to check that $\{u, u, x\}=\langle u, u\rangle x+\langle x, u\rangle u-\langle u, \bar{x}\rangle \bar{u}=\langle u, u\rangle x+\langle x, u\rangle u-\langle x, u\rangle u=x$, for all $x \in C_{4}$. This shows that $u$ is a unitary in $C_{4}$, and consequently $A \bar{\otimes} C_{4}$ is a $\mathrm{JBW}^{*}$-algebra whenever $A$ is a commutative von Neumann algebra.

Finally, assume that $C$ is a finite-dimensional Cartan factor and $A$ is a commutative von Neumann algebra. Then $A$ is isomorphic to $\bigoplus_{j \in J}^{\ell_{\infty}} L^{\infty}\left(\mu_{j}\right)$, where $\left(\mu_{j}\right)_{j \in J}$ is a family of finite (or, equivalently, probability) measures (cf. [72, §1.18]). Thus the $\mathrm{JBW}^{*}$-triple $A \bar{\otimes} C$ can be identified with $\bigoplus_{j \in J}^{\ell_{\infty}} L^{\infty}\left(\mu_{j}, C\right)$, (cf. [39, 41]). Let us observe that the remaining Cartan factors, that is, the exceptional Cartan factors of types 5 and 6 , are all finite-dimensional (they have dimensions 16 and 27, respectively).

Combining the arguments in the previous paragraphs we get the following representation of $\mathrm{JBW}^{*}$-triples.

Proposition 9.2. Let $M$ be any JBW*-triple. Then $M$ is (isometrically) JB*triple isomorphic to a $J B W^{*}$-triple of the form

$$
\left(\bigoplus_{k \in \Lambda_{1}}^{\ell_{\infty}} L^{\infty}\left(\mu_{k}, C_{k}\right)\right) \bigoplus^{\ell_{\infty}}\left(\bigoplus_{j \in \Lambda_{2}}^{\ell_{\infty}} L^{\infty}\left(\mu_{j}, D_{j}\right)\right) \bigoplus^{\ell_{\infty}} N \bigoplus^{\ell_{\infty}} p V,
$$

where

- $\left(\mu_{k}\right)_{k \in \Lambda_{1}}$ and $\left(\mu_{j}\right)_{j \in \Lambda_{2}}$ are two (possibly empty) families of probability measures; 
- Each $C_{k}$ is a Cartan factor of type 5 or 6 for any $k \in \Lambda_{1}$, and each $D_{j}$ is a finite-dimensional Cartan factor of type 2 with $\operatorname{dim}(H) \in \mathbb{N}$ odd for any $j \in \Lambda_{2}$;

- $N$ is a $J B W^{*}$-algebra;

- $V$ is a von Neumann algebra and $p \in V$ is a projection such that the triple $p V$ has no nonzero direct summand triple-isomorphic to a JBW*-algebra.

Moreover, such a representation is unique, in the sense that if $M$ admits two such representations, the respective four summands in one of them are triple-isomorphic to the respective four summands in the second one.

Thanks to the structure result in the previous proposition, the promised characterization of $\mathrm{JBW}^{*}$-triples is now stated in the following theorem.

Theorem 9.3. Let $M$ be a $J B W^{*}$-triple. Consider its representation provided by Proposition 9.2.

(a) $M$ is $\sigma$-finite if and only if the sets $\Lambda_{1}$ and $\Lambda_{2}$ are countable, the $J B W^{*}$-algebra $N$ is $\sigma$-finite and the projection $p$ is $\sigma$-finite.

(b) $M_{*}$ is $W C G$ if and only if $M$ is $\sigma$-finite.

(c) The following assertions are equivalent.

(i) $M_{*}$ is strongly $W C G$.

(ii) $M$ is $\sigma$-finite and, moreover, the projection $p$ is finite.

(iii) There is $\varphi \in M_{*} \backslash\{0\}$ such that the strong* topology on $B_{M}$ is generated by $\|\cdot\|_{\varphi}$.

(iv) There is a $\sigma$-finite tripotent $u \in M$ whose Peirce-2 subspace $M_{2}(u)$ is maximal with respect to inclusion.

Assertions $(a)$ and $(b)$ follow from 24] and [11, respectively. More concretely, the 'only if part'in $(a)$ is obvious; to see the 'if part' it is enough to use the known fact that exceptional Cartan factors are finite-dimensional and every $D_{j}$ is finitedimensional too, hence each of the summands $L^{\infty}\left(\mu_{\alpha}, C_{\alpha}\right)$ and $L^{\infty}\left(\mu_{j}, D_{j}\right)$ is $\sigma$ finite (cf. [24, Theorem 4.4]). Assertion (b) follows from [11, Theorem 1.1].

It remains to prove $(c)$. In view of $(b)$ we may restrict our attention to the $\sigma$-finite case. Let us observe that some implications in $(c)$ are easy at this point. Indeed, (iii) implies that the strong* topology on $B_{M}$ is metrizable, hence we get $(i i i) \Rightarrow(i)$. Further, $(i i i) \Rightarrow(i v)$ follows from Proposition 7.5(ii). Recall that a projection $p$ in a von Neumann algebra $V$ is finite if there is no partial isometry in $V$ with final projection $p$ and initial projection stricly less than $p$. The argument will follow after considering the individual summands in the representation. However, we first give the following corollary on $\mathrm{JBW}^{*}$-triples with separable predual. Note that while any separable Banach space is trivially $\mathrm{WCG}, c_{0}$ is an example of a separable space which is not strongly WCG by [73, Theorem 2.5]. A similar example cannot be a predual of a $\mathrm{JBW}^{*}$-triple.

Corollary 9.4. Let $M$ be a $J B W^{*}$-triple with separable predual $M_{*}$. Then $M_{*}$ is strongly $W C G$.

Proof. First observe that $M$ is $\sigma$-finite. Indeed, being separable, $M_{*}$ is WCG, thus $M$ is $\sigma$-finite by Theorem 9.3(b). (There is also an alternative way of proving this. Assume that $M_{*}$ is separable and fix $e \in M$ a complete tripotent. Then $M_{2}(e)_{*}$ is also separable. Since $M_{2}(e)$ is a $\mathrm{JBW}^{*}$-algebra, we can choose a countable family of normal states $\left\{\varphi_{n}: n \in \mathbb{N}\right\}$ which is norm-dense in the set of normal states of 
$M_{2}(e)$. Then $\sum_{n=1}^{\infty} \frac{1}{2^{n}} \varphi_{n}$ is a faithful normal state of $M_{2}(e)$. Therefore, $M_{2}(e)$ is $\sigma$-finite, so $e$ is $\sigma$-finite and $M$ is $\sigma$-finite as well.)

So, assume $M_{*}$ is separable and fix a representation of $M$ given by Proposition 9.2. It follows that $(p V)_{*}$ is separable as well. Without loss of generality there is no nonzero central projection in $V$ orthogonal to $p$ (if $z$ is such a projection, then $p V=p(1-z) V)$. We claim that in this case necessarily $V$ is $\sigma$-finite. Assume it is not the case. Then there is an uncountable family of pairwise orthogonal nonzero projections $\left(r_{\gamma}\right)_{\gamma \in \Gamma}$ in $V$. It follows from [77, Theorem V.1.8] that for each $\gamma \in \Gamma$ there is a nonzero partial isometry $u_{\gamma} \in V$ such that its initial projection $p_{i}\left(u_{\gamma}\right) \leq r_{\gamma}$ and its final projection $p_{f}\left(u_{\gamma}\right) \leq p$. Then clearly $u_{\gamma} \in p V$ for $\gamma \in \Gamma$. Fix $\varphi_{\gamma} \in(p V)_{*}$ of norm one with $u_{\gamma}=s\left(\varphi_{\gamma}\right)$. Since $\varphi_{\gamma}\left(u_{\gamma}\right)=1$ and for $\delta \neq \gamma$

$$
\varphi_{\gamma}\left(u_{\delta}\right)=\varphi_{\gamma} P_{2}\left(u_{\gamma}\right)\left(u_{\delta}\right)=0
$$

we see that $\left(\varphi_{\gamma}\right)_{\gamma \in \Gamma}$ is a 1-discrete set, contradicting the separability of $(p V)_{*}$.

Hence the strong* topology on $B_{V}$ is metrizable, so by Lemma 7.1(b) the same holds for $B_{p V}$, thus $(p V)_{*}$ is strongly WCG. Using Theorem 9.3 we now see that $p$ is finite and hence $M_{*}$ is strongly WCG as well.

To prove assertion $(c)$ in Theorem 9.3 we will describe the structure of all preHilbertian seminorms generating the strong* topology using Proposition 7.5 and some complements to that. We will do it first for $\sigma$-finite triples and then (in the next section) we shall discuss the general case. We start by analyzing the individual summands appearing in Proposition 9.2

9.1. $\mathbf{J B W}^{*}$-algebras. In the case of $\mathrm{JBW}^{*}$-algebras we can conclude by applying the existing literature. The desired conclusion is covered by the following proposition.

Proposition 9.5. Let $M$ be a $J B W^{*}$-algebra.

(a) Let $\left(e_{n}\right)$ be a sequence of $\sigma$-finite tripotents in $M$. Then there is a $\sigma$-finite projection $p \in M$ such that $M_{2}(p)$ contains $e_{n}$ for each $n \in \mathbb{N}$.

(b) Assume $M$ is not $\sigma$-finite. Then for each $\sigma$-finite projection $p \in M$ there is a $\sigma$-finite projection $q \in M$ such that $q>p$ (and hence $M_{2}(p) \varsubsetneqq M_{2}(q)$ ).

(c) The strong* topology on $B_{M}$ is metrizable if and only if $M$ is $\sigma$-finite. In this case it is metrizable by $\|\cdot\|_{\omega}$, where $\omega$ is any faithful normal state.

Proof. (a) This follows from Lemma 7.2( $d$ ) and [10, Lemma 3.5].

(b) This follows easily from the definitions. If $M$ is not $\sigma$-finite and $p$ is $\sigma$-finite, then $1-p \neq 0$, hence there is a $\sigma$-finite projection $r \in M_{2}(1-p)$. It is enough to take $q=p+r$.

(c) The 'if part' follows from Lemma 7.3. Conversely, assume that $B_{M}$ is metrizable in the strong* topology. Then there is a countable base of strong* neighborhoods of zero in $B_{M}$. It follows that the strong* topology on $B_{M}$ is generated by countably many seminorms. By (a) and Proposition 7.5(i) it is generated by one seminorm. By (b) and Proposition 7.5(ii) we deduce that $M$ is $\sigma$-finite.

9.2. Finite dimensional Cartan factors. In this subsection we shall deal with summands of the form $L^{\infty}(\mu, C)$ where $C$ is an exceptional Cartan factor (i.e., the Cartan factor of type 5 or 6 ) or a finite-dimensional Cartan factor of type 2 with $\operatorname{dim}(H) \in \mathbb{N}$ odd. We start with properties of a finite-dimensional JB*-triple. 
Let $E$ be a $\mathrm{JB}^{*}$-triple. Following the most employed notation, the symbol $\mathcal{U}(E)$ will stand for the set of all tripotents in $E$. We shall write $\mathcal{U}(E)^{*}$ for the set of all nonzero tripotents in $E$, and we shall employ the symbol $\mathcal{U}_{\max }(E)$ to denote the set of all complete tripotents in $E$. By Kaup's Riemann mapping theorem 52. Proposition 5.5], a linear bijection between $\mathrm{JB}^{*}$-triples $E$ and $F$ is a triple isomorphism if and only if it is an isometry. Henceforth, we denote by $\operatorname{Iso}(E, F)$ the set of all surjective isometries (equivalently, triple isomorphisms) from $E$ to $F$. We write $\operatorname{Iso}(E)=\operatorname{Iso}(E, E)$ for the set of all triple automorphisms of $E$.

Fix $\Phi \in \operatorname{Iso}(E)$. Then $\Phi$, being a $\mathrm{JB}^{*}$-triple automorphism, preserves all the triple structure. In particular, it maps tripotents to tripotents and complete tripotents to complete tripotents, that is,

$$
\Phi(E)\left(\mathcal{U}(E)^{*}\right)=\mathcal{U}(E)^{*} \text {, and } \Phi(E)\left(\mathcal{U}_{\max }(E)\right)=\mathcal{U}_{\text {max }}(E) .
$$

Moreover, the equality $\Phi\left(P_{j}(e)(x)\right)=P_{j}(\Phi(e))(\Phi(x))$ holds for every $e \in \mathcal{U}(E)$, $j \in\{0,1,2\}$ and $x \in E$. In particular, $\Phi\left(E_{2}(e)\right)=E_{2}(\Phi(e))$ and $\Phi$ is a (unital) $\mathrm{JB}^{*}$-algebra isomorphism of $E_{2}(e)$ onto $E_{2}(\Phi(e))$. Let us fix $e \in \mathcal{U}(E)$ and $\varphi \in E^{*}$ a functional satisfying $\varphi=\varphi P_{2}(e)$. Then $\varphi \circ \Phi^{-1}=\varphi \circ P_{2}(e) \circ \Phi^{-1}=\varphi \circ \Phi^{-1} \circ P_{2}(\Phi(e))$ and $\left.\left(\varphi \circ \Phi^{-1}\right)\right|_{E_{2}(\Phi(e))}=\left.\varphi\right|_{E_{2}(e)} \circ \Phi^{-1}$.

It is natural to ask about the orbit of a fixed $e \in \mathcal{U}_{\max }(E)$ under the group $\operatorname{Iso}(E)$. In general, $\operatorname{Iso}(E)(e)$ is not easy to be determined (cf. [13] and [54]). If $E$ is a finite-dimensional $\mathrm{JB}^{*}$-triple, then any two complete (maximal) tripotents in $E$ are interchanged by an element in $\operatorname{Iso}(E)$ (see [57, Theorem 5.3(b)]). This can be also seen by applying that $E$ being finite-dimensional implies that $E$ coincides with a finite $\ell_{\infty}$-sum of finite-dimensional Cartan factors, and it is known that on a finite-dimensional Cartan factor $C$ the group $\operatorname{Iso}(C)$ acts transitively on $\mathcal{U}_{\text {max }}(C)$. Therefore, for $e \in \mathcal{U}_{\max }(E)$ and $\operatorname{dim}(E)<\infty$ we have

$$
\operatorname{Iso}(E)(e)=\mathcal{U}_{\text {max }}(E) \text {. }
$$

Lemma 9.6. Let $E$ be a finite-dimensional $J B^{*}$-triple, let $e \in \mathcal{U}_{\text {max }}(E)$, and let $\varphi \in E_{*}$ be a norm-one functional such that $e=s(\varphi)$. Then the following assertions hold:

(a) For each $\Phi \in I s o(E)$ we have $\Phi(e) \in \mathcal{U}_{\max }(E)$ and $\Phi(e)=s\left(\varphi \circ \Phi^{-1}\right)$;

(b) $\mathcal{U}(E), \mathcal{U}(E)^{*}$, and $\mathcal{U}_{\max }(E)$ are compact subsets of $E$ and Iso $(E)$ is a compact subset of $B(E)$;

(c) There is a constant $\alpha>0$ such that for each $\Phi \in I$ Iso $(E)$ we have

$$
\alpha\|x\| \leq\|x\|_{\varphi \circ \Phi^{-1}} \leq\|x\|, \quad x \in E .
$$

$(d)$ There is a Borel measurable mapping $\theta: \mathcal{U}_{\max }(E) \rightarrow \operatorname{Iso}(E)$ such that $u=$ $\theta(u)(e)$ for each $u \in \mathcal{U}_{\max }(E)$.

Proof. Since $E$ is finite-dimensional, it is a $\sigma$-finite $\mathrm{JBW}^{*}$-triple, so $\varphi$ can be found.

(a) This was justified in (15).

(b) Since the triple product is jointly norm continuous, $\mathcal{U}(E)$ and $\mathcal{U}(E)^{*}=$ $\mathcal{U}(E) \backslash\{0\}$ are closed subsets of the closed unit ball and the unit sphere of $E$, respectively, so they are compact. Elements of $\operatorname{Iso}(E)$ are precisely (surjective) isometries, so $\operatorname{Iso}(E)$ is a closed subset of the unit sphere of $B(E)$, hence it is compact. 
We next consider the mapping $\Psi: \operatorname{Iso}(E) \rightarrow E$ defined by

$$
\Psi(\Phi)=\Phi(e), \quad \Phi \in \operatorname{Iso}(E) .
$$

It is clearly a continuous mapping and by $(a)$ it maps $\operatorname{Iso}(E)$ into $\mathcal{U}_{\text {max }}(E)$. We deduce from (16) that $\Psi$ is onto, so $\mathcal{U}_{\max }(E)$ is compact.

(c) For any $\Phi \in \operatorname{Iso}(E)$ and $x \in E$ we have (due to $(a)$ )

$$
\|x\|_{\varphi \circ \Phi^{-1}}^{2}=\left(\varphi \circ \Phi^{-1}\right)\{x, x, \Phi(e)\}=\varphi\left\{\Phi^{-1}(x), \Phi^{-1}(x), e\right\}=\left\|\Phi^{-1}(x)\right\|_{\varphi}^{2} .
$$

Since

$$
(x, \Phi) \mapsto\left\|\Phi^{-1}(x)\right\|_{\varphi}, \quad x \in S_{E}, \Phi \in \operatorname{Iso}(E)
$$

is a strictly positive continuous mapping on the compact space $S_{E} \times \operatorname{Iso}(E)$, it has some strictly positive minimum and maximum. Thus, the existence of the constant $\alpha$ easily follows. Clearly, $\|x\|_{\phi} \leq\|x\|$ for all $x \in M$ and every norm-one functional $\phi$ in $M_{*}$.

(d) The mapping $\Psi$ from the proof of $(b)$ is a continuous mapping of a compact metric space $\operatorname{Iso}(E)$ onto a compact metric space $\mathcal{U}_{\text {max }}(E)$, hence the inverse setvalued map $u \mapsto \Psi^{-1}(u)$ admits a Borel-measurable selection by the KuratowskiRyll-Nardzewski theorem (see [2, Theorem 18.13]).

The reader may already guess at this stage that the constant $\alpha>0$ given by Lemma 9.6(c) is directly linked to the dimension of the JB*-triple $E$. If we have a family $\left\{C_{k}: k \in \Lambda\right\}$ of finite-dimensional Cartan factors for which the $\operatorname{dim}\left(C_{k}\right)$ is uniformly bounded for all $k \in \Lambda$ (for example, a family of exceptional Cartan factors of types 5 and 6 ), then the constant $\alpha$ can be chosen to be valid for all $k \in \Lambda$.

Proposition 9.7. Let $E$ be a finite-dimensional $J B^{*}$-triple, and let $(\Omega, \Sigma, \mu)$ be a probability space. Consider the $J B W^{*}$-triple $M=L^{\infty}(\mu, E)$ (equipped with the pointwise triple product). Let $e, \varphi, \theta, \alpha$ be as in Lemma 9.6. Then the following assertions hold:

(a) An element $f \in M$ is a tripotent if and only if $f(\omega) \in \mathcal{U}(E) \mu$-almost everywhere;

(b) An element $f \in M$ is a complete tripotent if and only if $f(\omega) \in \mathcal{U}_{\max }(E)$ $\mu$-almost everywhere;

(c) Assume that $f \in M$ is a complete tripotent. Let

$$
v(\omega)=\varphi \circ \theta(f(\omega))^{-1}, \quad \omega \in \Omega .
$$

Then $v \in L^{1}\left(\mu, E_{*}\right)=L^{\infty}(\mu, E)_{*}$ and $s(v)=f$;

(d) Let $f$ and $v$ be as in $(c)$. Then

$$
\alpha\left(\int\|g(\omega)\|^{2} \mathrm{~d} \mu(\omega)\right)^{\frac{1}{2}} \leq\|g\|_{v} \leq\left(\int\|g(\omega)\|^{2} \mathrm{~d} \mu(\omega)\right)^{\frac{1}{2}}, \quad g \in M
$$

(e) The strong* topology on $B_{M}$ coincides with the topology generated by the norm $\|\cdot\|_{v}$ and also with the topology generated by the norm $g \mapsto\left(\int\|g(\omega)\|^{2} \mathrm{~d} \mu(\omega)\right)^{\frac{1}{2}}$.

Proof. Assertion (a) follows immediately from the fact that the triple product is defined pointwise. 
(b) Since the triple product is defined pointwise, we have, for a given tripotent $f \in M$,

$$
P_{0}(f)(g)(\omega)=P_{0}(f(\omega))(g(\omega)) \quad \mu \text {-a.e. }
$$

Hence, if $f(\omega) \in \mathcal{U}_{\max }(E) \mu$-almost everywhere, then clearly $P_{0}(f)=0$.

Conversely, assume that it is not true that $f(\omega) \in \mathcal{U}_{\max }(E) \mu$-almost everywhere. Since $\mathcal{U}_{\max }(E)$ is a closed set, there is a measurable set $A \subseteq \Omega$ of positive measure such $f(\omega) \notin \mathcal{U}_{\max }(E)$, for all $\omega \in A$.

For any $u \in \mathcal{U}(E)$ there is $u^{\prime} \in \mathcal{U}_{\max }(E)$ with $u \leq u^{\prime}$ (cf. [40, Lemma 3.12]). Moreover, the set

$$
\left\{\left(u, u^{\prime}\right) \in \mathcal{U}(E) \times \mathcal{U}_{\max }(E) ; u \leq u^{\prime}\right\}
$$

is closed, hence compact, and thus the set-valued mapping

$$
\mathcal{U}(E) \ni u \mapsto\left\{u^{\prime} \in \mathcal{U}_{\max }(E) ; u \leq u^{\prime}\right\}
$$

is upper-semicontinuous and compact-valued. By the Kuratowski-Ryll-Nardzewski theorem we find a Borel-measurable mapping $\zeta: \mathcal{U}(E) \rightarrow \mathcal{U}_{\max }(E)$ such that $u \leq \zeta(u)$ for $u \in \mathcal{U}(E)$.

Then the mapping $g=\zeta \circ f$ belongs to $M$ and

$$
P_{0}(f)(g)(\omega)=P_{0}(f(\omega))(g(\omega))=P_{0}(f(\omega))(\zeta(f(\omega))=\zeta(f(\omega))-f(\omega)
$$

which is nonzero on $A$. Thus $f$ is not complete.

$(c)$ By $(b)$ we know that $f(\omega) \in \mathcal{U}_{\max }(E) \mu$-almost everywhere, so the mapping $\omega \mapsto \theta(f(\omega))$ is a $\mu$-almost everywhere defined measurable mapping from $\Omega$ into Iso $(E)$. Since taking an inverse is a continuous transformation, we see that $v$ is a $\mu$-almost everywhere defined measurable mapping from $\Omega$ into $E_{*}$. Moreover, since $\|\varphi\|=1$ and elements of $\operatorname{Iso}(E)$ are isometries, $\|v(\omega)\|=1 \mu$-almost everywhere. Thus $v \in L^{1}\left(\mu, E_{*}\right)$ and $\|v\|=1$ (as $\mu$ is a probability measure). Moreover,

$$
\langle v, f\rangle=\int\langle v(\omega), f(\omega)\rangle \mathrm{d} \mu=\int \varphi \circ \theta(f(\omega))^{-1}(f(\omega)) \mathrm{d} \mu=\int \varphi(e) \mathrm{d} \mu=1 .
$$

Furthermore, assume that $h \in M_{2}(f)$ is positive with $\langle v, h\rangle=0$. Then $h(\omega)$ is a positive element of $E_{2}(f(\omega))$ for $\mu$-almost all $\omega \in \Omega$, hence $\theta(f(\omega))^{-1}(h(\omega))$ is a positive element of $E_{2}(e)$ for $\mu$-almost all $\omega$. Hence

$$
0=\langle v, h\rangle=\int\langle v(\omega), h(\omega)\rangle \mathrm{d} \mu=\int \varphi\left(\theta(f(\omega))^{-1}(h(\omega))\right) \mathrm{d} \mu,
$$

so $\varphi\left(\theta(f(\omega))^{-1}(h(\omega))\right)=0 \mu$-a.e. Since $\varphi$ is faithful on $E_{2}(e)$, we deduce that $\theta(f(\omega))^{-1}(h(\omega))=0 \mu$-a.e., so $h(\omega)=0 \mu$-a.e.

$(d)$ For any $g \in M$ we have

$$
\begin{aligned}
\|g\|_{v}^{2} & =\langle v,\{g, g, f\}\rangle=\int\langle v(\omega),\{g(\omega), g(\omega), f(\omega)\}\rangle \mathrm{d} \mu \\
& =\int\left\langle\varphi \circ \theta(f(\omega))^{-1},\{g(\omega), g(\omega), f(\omega)\}\right\rangle \mathrm{d} \mu=\int\|g(\omega)\|_{\varphi \circ \theta(f(\omega))^{-1}}^{2} \mathrm{~d} \mu
\end{aligned}
$$

so we can conclude by the choice of $\alpha$.

(e) For any tripotent $h \in M$ there is a complete tripotent $f \in M$ with $f \geq h$. For any complete tripotent $f$ let $v(f) \in M_{*}$ be as in $(c)$. By Proposition $7.5(i)$ the strong* topology on $B_{M}$ coincides with the topology generated by the seminorms 
$\|\cdot\|_{v(f)}, f \in M$ a complete tripotent. We deduce from $(d)$ that all these norms are equivalent to the norm $g \mapsto\left(\int\|g(\omega)\|^{2} \mathrm{~d} \mu(\omega)\right)^{\frac{1}{2}}$.

9.3. Triples of the form $p V$. It turns out that the analysis of this case is more complicated than the previous two cases. We shall employ an argument which is closely related to the notion of equivalence of projections and to the theory of types of von Neumann algebras (see, for example, [50]).

Given a von Neumann algebra $V$, two projections $p, q \in V$ are said to be equivalent (we write $p \sim q$ ) if there is a partial isometry in $V$ with initital projection $p$ and final projection $q$. Further, a projection $p$ is called finite if the only projection $q$ satisfying $q \leq p$ and $q \sim p$ is the projection $p$ itself. A projection which is not finite is called infinite. Finally, a projection $p$ is properly infinite if $z p$ is infinite for any central projection $z$ such that $z p \neq 0$.

For any projection $p \in V$ its central carrier is the smallest central projection $C_{p}$ satisfying $C_{p} p=p$. It is further known that there is a unique central projection $z \leq C_{p}$ such that $z p$ is properly infinite or zero and $(1-z) p=\left(C_{p}-z\right) p$ is finite. Indeed, if $p$ is finite, we take $z=0$, and if $p$ is infinite we may use [50, Proposition 6.3.7].

Henceforth, assume that we have a $\mathrm{JBW}^{*}$-triple of the form $p V$, where $V$ is a von Neumann algebra, and $p \in V$ is a projection. We may assume, without loss of generality, that $C_{p}=1$ (otherwise we may replace $V$ by $C_{p} V$ ). By the previous paragraph there is a central projection $z \in V$ such that $z p$ is properly infinite and $(1-z) p$ is finite. Then $p V=z p V \oplus(1-z) p V$, thus we discuss separately the cases in which $p$ is finite or properly infinite.

We begin with the following lemma on equivalence of projections.

Lemma 9.8. Let $V$ be a von Neumann algebra. Then the following assertions are true.

(a) Let $\left(p_{n}\right)$ be a sequence of properly infinite projections in $V$ which are all equivalent to one projection $q \in V$. Then the supremum of the sequence $\left(p_{n}\right)$ is also equivalent to $q$.

(b) Let $\left(p_{n}\right)$ be an increasing sequence of projections in $V$ with supremum $p$. If all the projections $p_{n}$ are equivalent to one projection $q$, then $p \sim q$ as well.

(c) Assume that $p_{1}, p_{2}$ are two equivalent projections in $V$. Then for any projection $q_{1} \geq p_{1}$ there is a projection $q_{2} \geq p_{2}$ such that $q_{1} \sim q_{2}$.

Proof. Assertion (a) is proved in [74, Lemma 3.2(1)].

(b) By [50, Proposition 6.2.8] we have $C_{p_{n}}=C_{q}$ for each $n \in \mathbb{N}$, hence $C_{p}=C_{q}$ by [49, Proposition 5.5.3]. So, denote by $c$ the common central carrier of all the projections in question.

Let $z \leq c$ be the central projection such that $z q$ is finite and $(c-z) q$ is properly infinite. Then $z p_{n} \sim z q$ for each $n \in \mathbb{N}$, so $z p_{n} \sim z p_{m}$ for $m, n \in \mathbb{N}$. Since $z p_{n}$ is finite for each $n$, we deduce that $z p_{n}=z p_{m}$ for each $m, n \in \mathbb{N}$, thus $z p=z p_{n}$ for $n \in \mathbb{N}$, hence $z p \sim z q$.

Further, $(c-z) p_{n} \sim(c-z) q$ for $n \in \mathbb{N}$. Since $(c-z) q$ is properly infinite, the projections $(c-z) p_{n}$ are properly infinite as well. Thus by $(a)$ we deduce that $(c-z) p \sim(c-z) q$, hence by [50, Proposition 6.2.2] $p \sim q$. 
(c) By the comparability theorem [77, Theorem V.1.8] for the pair of projections $q_{1}-p_{1}$ and $1-p_{2}$, there is a central projection $z$ such that

- $z\left(q_{1}-p_{1}\right)$ is equivalent to some projection $r \leq z\left(1-p_{2}\right)$, and

- $(1-z)\left(1-p_{2}\right)$ is equivalent to some projection $s \leq(1-z)\left(q_{1}-p_{1}\right)$.

By [50, Proposition 6.2.2] we get that $z q_{1}=z p_{1}+z\left(q_{1}-p_{1}\right)$ is equivalent to $r+z p_{2}$ and, moreover, $1-z=(1-z) p_{2}+(1-z)\left(1-p_{2}\right)$ is equivalent to $(1-z) p_{1}+s \leq(1-z) q_{1}$. But this means that $(1-z) q_{1}$ is equivalent to $1-z$ (by [50, Proposition 6.2.4]). Finally, one can take $q_{2}=r+z p_{2}+1-z$.

We consider first the case in which $p$ is finite.

Lemma 9.9. Let $V$ be a von Neumann algebra and $p \in V$ be a finite and $\sigma$-finite projection such that $p \neq 1$. Consider the $J B W^{*}$-triple $M=p V$.

(a) There is $\tau \in M_{*}$ such that $s(\tau)=p, \tau(p)=1$ and $\left.\tau\right|_{p V p}$ is a trace.

(b) Let $u \in M$ be a complete tripotent. Then $u$ can be extended to a unitary operator $\tilde{u} \in V$. Moreover, the functional

$$
\tau_{u}(x)=\tau\left(x \tilde{u}^{*}\right), \quad x \in M,
$$

belongs to $M_{*}, s\left(\tau_{u}\right)=u$ and

$$
\frac{1}{\sqrt{2}} \sqrt{\tau\left(p x x^{*} p\right)} \leq\|x\|_{\tau_{u}} \leq \sqrt{\tau\left(p x x^{*} p\right)}, \quad x \in M .
$$

(c) The strong* topology on $B_{M}$ is generated by the norm $\|\cdot\|_{\tau}$ and also by the norm

$$
x \mapsto \sqrt{\tau\left(p x x^{*} p\right)}
$$

Proof. (a) Since $p V p$ is a finite and $\sigma$-finite von Neumann algebra with unit $p$, it admits a normal finite faithful trace $\tau$ with $\tau(p)=1$. Indeed, such a trace can be obtained by composition of the standard canonical center valued trace on $p V p$ (see [77. Theorem V.2.6]) with any norm-one faithful positive normal functional on the center of $p V p$. Then $\tau \circ P_{2}(p)$ (i.e., the mapping $x \mapsto \tau(x p)$ ) is an extension of $\tau$ to $p V$. Clearly $p=s\left(\tau \circ P_{2}(p)\right)$, hence it is enough to denote the composition again by $\tau$.

(b) Let $u \in M$ be a complete tripotent. Then it is a partial isometry in $V$ with final projection $p_{f}(u) \leq p$. Since $p_{f}(u)$ is finite $u$ can be extended to a unitary operator $\tilde{u} \in V$ (by [77, Proposition V.1.38]). Moreover, observe that the final projection $p_{f}(u)$ must coincide with $p$. Indeed, $p \tilde{u} \in M$ and, since $u$ is complete,

$$
0=P_{0}(u)(p \tilde{u})=\left(p-p_{f}(u)\right) p \tilde{u}\left(1-p_{i}(u)\right)=\left(p-p_{f}(u)\right) \tilde{u}\left(1-p_{i}(u)\right) .
$$

Since $p$ is finite, we get $p_{i}(u) \neq 1$. Moreover, $\tilde{u}$ maps the range of $1-p_{i}(u)$ onto the range of $1-p_{f}(u)$, which contains the range of $p-p_{f}(u)$. It follows that $p_{f}(u)=p$.

Set $q=p_{i}(u)=u^{*} u$ and consider the operator $v: M \rightarrow M$ defined by

$$
v(x)=x \tilde{u}^{*}, \quad x \in M .
$$

Then $v$ is a surjective isometry. Hence it is a triple isomorphism (this can be also easily checked directly), in particular, it is a weak*-to-weak* homeomorphism. 
Since $v(u)=p$, we deduce that $s(\tau \circ v)=u$. Thus,

$$
\begin{aligned}
\|x\|_{\tau \circ v}^{2} & =(\tau \circ v)(\{x, x, u\})=\tau(\{v(x), v(x), v(u)\})=\tau\left(\left\{x \tilde{u}^{*}, x \tilde{u}^{*}, p\right\}\right) \\
& =\frac{1}{2} \tau\left(p x \tilde{u}^{*} \tilde{u} x^{*} p+p \tilde{u} x^{*} x \tilde{u}^{*} p\right)=\frac{1}{2} \tau\left(p x x^{*} p+u x^{*} x u^{*}\right) \\
& =\frac{1}{2}\left(\tau\left(p x x^{*} p\right)+\tau\left(\left(u x^{*} p\right)\left(p x u^{*}\right)\right)\right)=\frac{1}{2}\left(\tau\left(p x x^{*} p\right)+\tau\left(\left(p x u^{*}\right)\left(u x^{*} p\right)\right)\right. \\
& =\frac{1}{2}\left(\tau\left(p x x^{*} p\right)+\tau\left(p x q x^{*} p\right)\right) .
\end{aligned}
$$

Since $q \leq 1$, we deduce $p x q x^{*} p \leq p x x^{*} p$, and thus

$$
\frac{1}{2} \tau\left(p x x^{*} p\right) \leq\|x\|_{\tau \circ \mathrm{v}}^{2} \leq \tau\left(p x x^{*} p\right) .
$$

Since $\tau_{u}=\tau \circ v$, the proof is completed.

(c) It follows from (b) combined with Proposition $7.5(i)$ that the strong* topology on $B_{M}$ coincides with the topology generated by the norms $\|\cdot\|_{\tau_{u}}$, where $u \in M$ is a complete tripotent. By a further application of $(b)$ we see that all these norms are equivalent to the one given in $(c)$.

We finally consider the case in which $p$ is properly infinite.

Proposition 9.10. Let $V$ be a von Neumann algebra and let $p \in V$ be a $\sigma$-finite properly infinite projection. Consider the $J B W^{*}$-triple $M=p V$. Assume that $M$ contains no nonzero direct summand triple-isomorphic to a JBW*-algebra. Then the following assertions hold:

(a) $V$ is not $\sigma$-finite;

(b) A tripotent $u \in M$ is complete if and only if its final projection equals p;

(c) Let $\left(u_{n}\right)$ be a sequence of complete tripotents in $M$. Then there is a complete tripotent $u \in M$ such that $M_{2}\left(u_{n}\right) \subseteq M_{2}(u)$ for each $n \in \mathbb{N}$;

(d) If $u \in M$ is a complete tripotent, then there is a complete tripotent $v \in M$ such that $M_{2}(u) \varsubsetneqq M_{2}(v)$;

(e) The strong* topology on $B_{M}$ is not metrizable.

Proof. (a) If $V$ is $\sigma$-finite, then $p \sim 1$ (as $C_{p}=1$ and $p$ is purely infinite), thus $M=p V$ would be triple-isomorphic to a $\mathrm{JBW}^{*}$-algebra (given a partial isometry $u$ with $u u^{*}=p$ and $u^{*} u=1$, the mapping $x \mapsto x u^{*}$ is a surjective isometry from $M$ onto $p V p)$.

(b) The 'if part' is clear. Let us prove the 'only if part'. Assume $p_{f}(u)<p$. By (a) we get $p_{i}(u)<1$. Thus $p-p_{f}(u)$ and $1-p_{i}(u)$ are two nonzero projections in $V$, thus it follows easily from the comparability theorem [77, Theorem V.1.8] that there are two nonzero projections $q_{1} \leq 1-p_{i}(u)$ and $q_{2} \leq p-p_{f}(u)$ which are equivalent. Fix a partial isometry $v \in V$ with initial projection $q_{1}$ and final projection $q_{2}$. Then $v \in M$ and

$$
P_{0}(u)(v)=\left(p-p_{f}(u)\right) v\left(1-p_{i}(u)\right) \neq 0
$$

as the range of $1-p_{i}(u)$ contains the range of $q_{1}, v$ maps it isometrically to the range of $q_{2}$ which is contained in the range of $p-p_{f}(u)$.

$(c)$ By $(b)$ we know that $p_{f}\left(u_{n}\right)=p$ for each $n$. So, $p \sim p_{i}\left(u_{n}\right)$ for each $n \in \mathbb{N}$. If we set $q=\sup _{n} p_{i}\left(u_{n}\right)$, Lemma 9.8(a) yields $p \sim q$. Then $u$ can be any partial isometry with initial projection $q$ and final projection $p$. 
$(d)$ By $(b)$ we know that $p_{f}(u)=p$. Since $p_{i}(u)<1$ (by $(a)$ ), we can find a $\sigma$-finite projection $q>p_{i}(u)$. Then $q$ is properly infinite, and hence $p \sim q$. Then $v$ can be any partial isometry with initial projection $q$ and final projection $p$.

(e) Assume that the restriction of the strong* topology to $B_{M}$ is metrizable. Then it is first countable, hence generated by countably many of the defining seminorms. Then $(c)$ and $(d)$ together with Proposition $7.5(i)$ yield a contradiction.

9.4. The case of a general $\sigma$-finite $\mathbf{J B W}^{*}$-triple. We are now ready to prove assertion $(c)$ of Theorem 9.3 . We will do it by proving the following two propositions (the final proof follows them).

Proposition 9.11. Assume that $M$ is a nontrivial $J B W^{*}$-triple of the form

$$
\left(\bigoplus_{k \in \Lambda}^{\ell_{\infty}} L^{\infty}\left(\mu_{k}, C_{k}\right)\right) \bigoplus^{\ell_{\infty}} N \bigoplus^{\ell_{\infty}} p V
$$

where

- $\Lambda$ is a (possibly empty) countable set;

- $\left(\mu_{k}\right)_{k \in \Lambda}$ is a (possibly empty) family of probability measures;

- Each $C_{k}$ is a Cartan factor of type 5 or 6 or a finite-dimensional Cartan factor of type 2 in $B\left(H_{k}\right)$ with $\operatorname{dim}\left(H_{k}\right)$ odd;

- $N$ is a (possibly trivial) $\sigma$-finite $J B W^{*}$-algebra;

- $V$ is a (possibly trivial) von Neumann algebra and $p \in V$ is a finite $\sigma$-finite projection such that the triple $p V$ has no nonzero direct summand triple-isomorphic to a $J B W^{*}$-algebra.

Fix a faithful normal state $\phi_{3} \in N_{*}$. Let $\tau \in(p V)_{*}$ be as in Lemma 9.9 (a). Then the following statements hold:

(a) We can regard $\phi_{3}$ as an element in $M_{*}$ satisfying that the strong* topology on $B_{N}$ is metrizable by the norm $\left.\|\cdot\|_{\phi_{3}}\right|_{N}$;

(b) We can regard $\tau$ as an element in $M_{*}$ satisfying that the strong* topology on $B_{(p V)}$ is metrizable by the norm $\left.\|\cdot\|_{\tau}\right|_{p V}$;

(c) Let $C=\bigoplus_{k \in \Lambda}^{\ell_{\infty}} L^{\infty}\left(\mu_{k}, C_{k}\right)$. Fix any $\varphi \in C_{*} \backslash\{0\}$ such that $s(\varphi) \in \mathcal{U}_{\max }(C)$. Then the norm $\|\cdot\|_{\varphi}$ is equivalent to the norm

$$
\left(a_{k}\right)_{k \in \Lambda} \mapsto\left(\sum_{n=1}^{\infty} 4^{-n} \int\left\|a_{k_{n}}\right\|^{2} \mathrm{~d} \mu_{k_{n}}\right)^{\frac{1}{2}}
$$

on bounded sets of $C$ (where $\left(k_{n}\right)$ is an enumeration of $\Lambda$ ). The strong* topology on $B_{C}$ is metrized by the norm displayed above;

(d) The strong* topology on $B_{M}$ is metrized by the norm $\|\cdot\|_{\tau+\phi_{3}+\varphi}$ (where the functional $\varphi$ from $(c)$ is considered as an element of $M_{*}$ ) which is equivalent to the norm

$$
\left\|\left(\left(a_{k}\right)_{k \in \Lambda}, x, y\right)\right\|^{2}=\left(\sum_{n=1}^{\infty} \frac{1}{4^{n}} \int\left\|a_{k_{n}}\right\|^{2} \mathrm{~d} \mu\right)+\|x\|_{\phi_{3}}^{2}+\tau\left(p y^{*} y p\right) .
$$

Proof. $(a)$ and $(b)$ are proved in Lemmata $7.3(b)$ and 9.9, respectively.

(c) Fix any $\varphi \in C_{*} \backslash\{0\}$ such that $s(\varphi)=\left(f_{k}\right)_{k \in \Lambda} \in \mathcal{U}_{\max }(C)$. 
Fix $k \in \Lambda$. Then $f_{k}$ is a maximal tripotent in $L^{\infty}\left(\mu_{k}, C_{k}\right)$, hence we can fix $h_{k} \in L^{1}\left(\mu_{k},\left(C_{k}\right)_{*}\right)$ provided by Proposition 9.7(c). Let $\left(k_{n}\right)$ be an enumeration of $\Lambda$ and set

$$
\phi_{1}\left(\left(a_{k}\right)_{k \in \Lambda}\right)=\sum_{n=1}^{\infty} 2^{-n}\left\langle h_{k_{n}}, a_{k_{n}}\right\rangle, \quad\left(\left(a_{k}\right)_{k \in \Lambda} \in C\right) .
$$

Clearly, $s\left(\phi_{1}\right)=\left(f_{k}\right)_{k \in \Lambda}=s(\varphi)$, so $\|\cdot\|_{\phi_{1}}$ and $\|\cdot\|_{\varphi}$ are equivalent on $B_{C}$ by Proposition 7.5. By Proposition 9.7 the norm $\|\cdot\|_{h_{k}}$ is equivalent to the norm

$$
f \mapsto\left(\int\|f\|^{2} \mathrm{~d} \mu_{k}\right)^{1 / 2}
$$

on the unit ball of $L^{\infty}\left(\mu_{k}, C_{k}\right)$ for each $k \in \Lambda$. Hence, the norm

$$
\left\|\left(a_{k}\right)_{k \in \Lambda}\right\|_{\phi_{1}}=\left(\sum_{n=1}^{\infty} 4^{-n}\left\|a_{k_{n}}\right\|_{h_{k_{n}}}^{2}\right)^{1 / 2}
$$

is equivalent on $B_{C}$ to the norm

$$
\left(a_{k}\right)_{k \in \Lambda} \mapsto\left(\sum_{n=1}^{\infty} 4^{-n} \int\left\|a_{k_{n}}\right\|^{2}\right)^{1 / 2} .
$$

Indeed, both norms are well defined. Moreover, a bounded sequence $\left(\left(a_{k}^{j}\right)_{k \in \Lambda}\right)_{j=1}^{\infty}$ converges to zero in the first norm if and only if

$$
\left\|a_{k}^{j}\right\|_{h_{k}} \stackrel{j}{\rightarrow} 0 \text { for each } k \in \Lambda,
$$

which takes place if and only if

$$
\int\left\|a_{k}^{j}\right\|^{2} \mathrm{~d} \mu_{k} \stackrel{j}{\rightarrow} 0 \text { for each } k \in \mathbb{N},
$$

which is in turn equivalent to the convergence to zero in the second norm.

Finally, it follows from Proposition 7.5 that the strong* topology on $B_{C}$ is generated by the mentioned norm.

Lastly, statement $(d)$ follows from the previous statements.

The remaining case is treated in our next result.

Proposition 9.12. Assume that $M$ is a $J B W^{*}$-triple of the form

$$
\left(\bigoplus_{k \in \Lambda}^{\ell_{\infty}} L^{\infty}\left(\mu_{k}, C_{k}\right)\right) \bigoplus^{\ell_{\infty}} N \bigoplus^{\ell_{\infty}} p V \bigoplus^{\ell_{\infty}} q W,
$$

where

- $\Lambda$ is a (possibly empty) countable set;

- $\left(\mu_{k}\right)_{k \in \Lambda}$ is a (possibly empty) family of probability measures;

- Each $C_{k}$ is a Cartan factor of type 5 or 6 or a finite-dimensional Cartan factor of type 2 in $B\left(H_{j}\right)$ with $\operatorname{dim}\left(H_{j}\right)$ odd;

- $N$ is a (possibly trivial) $\sigma$-finite $J B W^{*}$-algebra;

- $V$ is a (possibly trivial) von Neumann algebra and $p \in V$ is a finite $\sigma$-finite projection such that the triple $p V$ has no nonzero direct summand triple-isomorphic to a $J B W^{*}$-algebra; 
- $W$ is a nontrivial von Neumann algebra and $q \in W$ is a properly infinite $\sigma$ finite projection such that the triple $q W$ has no nonzero direct summand tripleisomorphic to a $J B W^{*}$-algebra.

Then the strong* topology on $B_{M}$ is not metrizable.

Proof. Proposition 9.10( $(e)$ assures that the strong* topology on $B_{q W}$ is not metrizable, and the desired conclusion follows from Lemma 7.1 (b).

Proof of Theorem 9.3 (c). (ii) $\Rightarrow$ (iii) This follows from Proposition 9.11

$($ iii $) \Rightarrow($ i $)$ This follows from Proposition 7.9 and [73, Theorem 2.1] as explained above.

$(i) \Rightarrow(i i)$ Assume $p$ is not finite. By Proposition 9.12 the strong* topology on $B_{M}$ is not metrizable. Hence $M_{*}$ is not strongly WCG by Proposition 7.9 and [73, Theorem 2.1].

$($ iii $) \Rightarrow(i v)$ This follows from Proposition 7.5 (ii).

$(i v) \Rightarrow(i i)$ Assume (iv) holds but $p$ is not finite. It follows from (iv) that $M$ is $\sigma$-finite, hence $M$ has the form from Proposition 9.12 Let $u=\left(\left(a_{k}\right),\left(b_{j}\right), x, v, w\right)$ be any tripotent in $M$. Then $w$ is a tripotent in $q W$. It follows from Proposition 9.10 that there is a tripotent $\tilde{w} \in q W$ with $(q W)_{2}(w) \varsubsetneqq(q W)_{2}(\tilde{w})$. Set $\tilde{u}=\left(\left(a_{k}\right),\left(b_{j}\right), x, v, \tilde{w}\right)$. Then $\tilde{u}$ is a tripotent in $M$ and $M_{2}(u) \varsubsetneqq M_{2}(\tilde{u})$.

Remark 9.13. It follows from the analysis of the individual cases in this section that any $\sigma$-finite $\mathrm{JBW}^{*}$-triple can be expressed as a direct sum of (countably many) summands of three different types.

Type $1-\mathbf{J B W}^{*}$-algebra: If $N$ is a $\sigma$-finite $\mathrm{JBW}^{*}$-algebra, it admits a unit, i.e., a ( $\sigma$-finite) unitary element. Then $N_{2}\left(1_{N}\right)=N$, hence the Peirce-2 subspace is the largest possible.

Type $2-L^{\infty}(\mu, C)$ or $p V$ with $p$ finite: Assume $M=L^{\infty}(\mu, C)$, where $\mu$ is a probability measure and $C$ is a finite-dimensional Cartan factor without a unitary element, or $M=p V$, where $V$ is a von Neumann algebra and $p \in V$ is a finite $\sigma$-finite projection such that $M$ has no direct summand isomorphic to a $\mathrm{JBW}^{*}$-algebra. Then there are tripotents whose Peirce-2 subspaces are maximal with respect to inclusion, but mutually different. But all the norms $\|\cdot\|_{\varphi}$, where $s(\varphi)$ is such a tripotent, are equivalent on bounded sets.

Type $3-p V$ for $p$ properly infinite: Assume that $M=p V$, where $V$ is a von Neumann algebra and $p \in V$ is a properly infinite $\sigma$-finite projection such that $M$ has no direct summand isomorphic to a $\mathrm{JBW}^{*}$-algebra. Then the family of Peirce- 2 subspaces $M_{2}(u), u \in \mathcal{U}(M)$, is upwards $\sigma$-directed by inclusion and has no maximal element.

In the next section we give some consequences of this trichotomy to the structure of general (not necessarily $\sigma$-finite) $\mathrm{JBW}^{*}$-triples.

\section{On SEMINORMS GENERATING THE STRONG* TOPOLOGY}

In this section we provide a characterization of the natural ordering of the seminorms generating the strong* topology for a $\mathrm{JBW}^{*}$-triple, which is defined by inclusion of the respective topologies on the unit ball. The case of $\sigma$-finite triples is covered by Propositions 9.11 and 9.12, here we deal with general triples. The promised result is contained in the following theorem. 
Theorem 10.1. Let $M$ be a JBW*-triple. Then it can be represented in the form

$$
M=\bigoplus_{\alpha \in \Lambda} L^{\infty}\left(\mu_{\alpha}, C_{\alpha}\right) \oplus N \oplus p V \oplus q W
$$

where

- $\Lambda$ is an arbitrary (possibly empty) set;

- $\mu_{\alpha}$ is a probability measure and $C_{\alpha}$ is a finite-dimensional Cartan factor not containing a unitary element for any $\alpha \in \Lambda$;

- $N$ is a (possibly trivial) $J B W^{*}$-algebra;

- $V$ is a (possibly trivial) von Neumann algebra and $p \in V$ is a finite projection such that the triple $p V$ has no nonzero direct summand triple-isomorphic to a $J B W^{*}$-algebra;

- $p=\sum_{j \in J} p_{j}$, where $\left(p_{j}\right)_{j \in J}$ is an orthogonal family of (finite) $\sigma$-finite projections in the center of $p V p$;

- $W$ is a (possibly trivial) von Neumann algebra and $q \in W$ is a properly infinite projection such that the triple $q W$ has no nonzero direct summand tripleisomorphic to a $J B W^{*}$-algebra.

For an element

$$
\boldsymbol{h}=\left(\left(f_{\alpha}(\boldsymbol{h})\right)_{\alpha \in \Lambda}, x(\boldsymbol{h}), v(\boldsymbol{h}), w(\boldsymbol{h})\right)=\left(\left(f_{\alpha}\right)_{\alpha \in \Lambda}, x, v, w\right) \in M
$$

we denote

$$
\operatorname{spt}_{1} \boldsymbol{h}=\left\{\alpha \in \Lambda ; f_{\alpha} \neq 0\right\}, \quad \operatorname{spt}_{2} \boldsymbol{h}=\left\{j \in J ; p_{j} v \neq 0\right\} .
$$

Further, set

$$
\begin{aligned}
\mathcal{T}(M)=\left\{\boldsymbol{h}=\left(\left(f_{\alpha}\right)_{\alpha \in \Lambda}, x, v, w\right) \in M ; \mathrm{spt}_{1} \boldsymbol{h}, \mathrm{spt}_{2} \boldsymbol{h}\right. \text { are countable, } \\
\quad \forall \alpha \in \mathrm{spt}_{1} \boldsymbol{h}: f_{\alpha} \text { is a complete tripotent in } L^{\infty}\left(\mu_{\alpha}, C_{\alpha}\right), \\
\quad x \text { is a } \sigma \text {-finite projection in } N, v v^{*}=\sum_{j \in \mathrm{spt}_{2} \boldsymbol{h}} p_{j}, \\
\\
\left.\quad w w^{*} \text { is a properly infinite } \sigma \text {-finite projection below } q\right\} .
\end{aligned}
$$

Then the following assertions hold:

(a) The elements of $\mathcal{T}(M)$ are $\sigma$-finite tripotents in $M$. Moreover, for any $\sigma$-finite tripotent $\boldsymbol{g} \in M$ there is $\boldsymbol{h} \in \mathcal{T}(M)$ with $M_{2}(\boldsymbol{g}) \subseteq M_{2}(\boldsymbol{h})$.

(b) Let $\varphi, \psi \in M_{*} \backslash\{0\}$ such that the support tripotents of these functionals belong to $\mathcal{T}(M)$. Set $\boldsymbol{h}=s(\varphi)$ and $\boldsymbol{g}=s(\psi)$. Then $\|\cdot\|_{\varphi}$ is weaker than $\|\cdot\|_{\psi}$ on $B_{M}$ if and only if the following assertions hold:

○ $\operatorname{spt}_{1} \boldsymbol{h} \subseteq \mathrm{spt}_{1} \boldsymbol{g}$ and $\mathrm{spt}_{2} \boldsymbol{h} \subseteq \mathrm{spt}_{2} \boldsymbol{g}$;

$\circ x(\boldsymbol{h}) \leq x(\boldsymbol{g})$ as projections in $N$;

$\circ\|\cdot\|_{\varphi}$ is weaker than $\|\cdot\|_{\psi}$ on $B_{q W}$.

Before proving this theorem let us formulate some consequences.

Corollary 10.2. Let $M$ be a JBW*-triple. Given a sequence $\left(\varphi_{n}\right)$ in $M_{*} \backslash\{0\}$, there is $\psi \in M_{*} \backslash\{0\}$, such that $\|\cdot\|_{\varphi_{n}}$ is weaker than $\|\cdot\|_{\psi}$ on $B_{M}$ for each $n \in \mathbb{N}$, i.e., the family of topologies on $B_{M}$ generated by the seminorms $\|\cdot\|_{\varphi}, \varphi \in M_{*} \backslash\{0\}$ is upwards $\sigma$-directed by inclusion.

The proof of this corollary will use one of the lemmata below, so we postpone the the end of the section. 
Corollary 10.3. Let $M$ be a $J B W^{*}$-triple and $L \subseteq M_{*}$ any weakly compact set. Then there is $\varphi \in M_{*}$ such that for any $\varepsilon>0$ there is $n \in \mathbb{N}$ satisfying $L \subseteq$ $n K(\varphi)+\varepsilon B_{M_{*}}$.

Proof. We will imitate the proof of Proposition 7.11 using the same notation. The seminorm $q_{L}$ is Mackey continuous, so $\left.q_{L}\right|_{B_{M}}$ is strong*-continuous. Hence, given $m \in \mathbb{N}$, there are $\varphi_{1}^{m}, \ldots, \varphi_{k_{m}}^{m} \in M_{*} \backslash\{0\}$ and $\delta_{m}>0$ such that

$$
\left\{x \in B_{M} ;\|x\|_{\varphi_{j}^{m}} \leq \delta_{m} \text { for } j=1, \ldots, k_{m}\right\} \subseteq\left\{x \in B_{M} ; q_{L}(x) \leq \frac{1}{m}\right\} .
$$

By Corollary 10.2 there is $\varphi \in M_{*} \backslash\{0\}$ such that $\|\cdot\|_{\varphi_{j}^{m}}$ is weaker than $\|\cdot\|_{\varphi}$ on $B_{M}$ for each $m \in \mathbb{N}$ and $j=1, \ldots, k_{m}$. Since $\|\cdot\|_{\varphi}$ is on $B_{M}$ equivalent to $\|\cdot\|_{\varphi}^{K}=q_{K(\varphi)}$ (by Lemma $7.10(c, d)$ ) we deduce that for each $m \in \mathbb{N}$ there is $\eta_{m}>0$ such that

$$
\left\{x \in B_{M} ; q_{K(\varphi)}(x) \leq \eta_{m}\right\} \subseteq\left\{x \in B_{M} ; q_{L}(x) \leq \frac{1}{m}\right\} .
$$

The calculation of polars (see the proof of Proposition 7.11) shows that

$$
L \subseteq \frac{1}{m \eta_{m}} K(\varphi)+\frac{2}{m} B_{M_{*}} .
$$

This completes the proof.

Now we proceed with the proof of Theorem 10.1. This will be done in several steps.

Let us start by explaining the existence of the respective representation. Let $M$ be any JBW*-triple. By Proposition $9.2 M$ can be represented as

$$
M=\bigoplus_{\alpha \in \Lambda} L^{\infty}\left(\mu_{\alpha}, C_{\alpha}\right) \oplus N \oplus s U,
$$

where $\Lambda$ is a set, $\mu_{\alpha}$ is a probability measure and $C_{\alpha}$ is a finite-dimensional Cartan factor without unitary element for each $\alpha \in \Lambda, N$ is a $\mathrm{JBW}^{*}$-algebra, $U$ is a von Neumann algebra, and $s \in U$ is a projection such that $s U$ admits no nonzero direct summand isomorphic to a $\mathrm{JBW}^{*}$-algebra. We may assume without loss of generality that $C_{s}=1_{U}$. By [50, Proposition 6.3.7] there is a unique central projection $z \in U$ such that $z s$ is poperly infinite or zero and $(1-z) s$ is finite, hence $s U=z s U \oplus(1-z) s U$. Take $W=z U, q=z s, V=(1-z) U, p=(1-z) s$. Then we have the representation of the form from Theorem 10.1 where $p$ is finite and $q$ properly infinite. Finally, the existence of the relevant decomposition of $p$ follows from [77, Corollary V.2.9].

We continue by proving assertion (a). It is clear that all the elements of $\mathcal{T}(M)$ are $\sigma$-finite tripotents. To prove the second statement we will use two lemmata.

Lemma 10.4. Let $V$ be a von Neumann algebra, $u \in V$ a $\sigma$-finite tripotent and $\left(r_{j}\right)_{j \in J}$ an orthogonal family of projections. Then the sets

$$
\left\{j \in J ; r_{j} u \neq 0\right\} \text { and }\left\{j \in J ; u r_{j} \neq 0\right\}
$$

are countable.

Proof. Note that $u$, being a tripotent, is a partial isometry with initial projection $p_{i}(u)=u^{*} u$ and final projection $p_{f}(u)=u u^{*}$. Moreover, since $u$ is $\sigma$-finite, both $p_{i}(u)$ and $p_{f}(u)$ are $\sigma$-finite. Further, it is clear that $r_{j} u \neq 0$ if and only if $r_{j} p_{f}(u) \neq$ 
0 , and that $u r_{j} \neq 0$ if and only if $p_{i}(u) r_{j} \neq 0$. We can therefore assume, without loss of generality, that $u$ is a projection.

So, assume $u$ is a projection. Now consider two orthogonal families of cyclic projections in $V$ with sum equal to 1 , say $\left(q_{\gamma}\right)_{\gamma \in \Gamma}$ and $\left(s_{\delta}\right)_{\delta \in \Delta}$, such that $u$ is the sum of a subfamily of the first one and $r_{j}$ is the sum of a subfamily of the second one for each $j \in J$. The existence of these families follows easily from [49, Proposition 5.5.9].

Since $\left\{\gamma \in \Gamma ; q_{\gamma} u \neq 0\right\}$ is countable, 9, Proposition 4.1] implies that both sets $\left\{\delta \in \Delta ; u s_{\delta} \neq 0\right\}$ and $\left\{\delta \in \Delta ; s_{\delta} u \neq 0\right\}$ are countable. Now the assertion easily follows.

Lemma 10.5. Let $V$ be a von Neumann algebra and let $p \in V$ be a properly infinite projection. Then for any $\sigma$-finite projection $q \leq p$ there is a properly infinite $\sigma$-finite projection $r$ such that $q \leq r \leq p$.

Proof. Without loss of generality we can assume that $p=1$. According to assumption $V$ is properly infinite. Therefore there is a sequence $\left(q_{n}\right)$ of mutually orthogonal projections such that $\sum_{n} q_{n}=1$ and $q_{n} \sim 1$ for each $n$ (see e.g. Proposition 4.12, page 97 in 76]). Therefore, there are projections $r_{n}^{\prime}$ with $r_{n}^{\prime} \leq q_{n}$ and $q \sim r_{n}^{\prime}$ for each $n$ (cf. Lemma 9.8 (c)). Then $r^{\prime}=\sum_{n} r_{n}^{\prime}$ is a properly infinite $\sigma$-finite projection (cf. [76, Proposition 4.12]) to which $q$ is subequivalent (see Lemma 9.8). Now by Lemma 9.8 $(c)$ there is a projection $r \geq q$ with $r \sim r^{\prime}$. This projection is $\sigma$-finite and properly infinite.

Now we are ready to prove the second statement of assertion $(a)$. Let

$$
\boldsymbol{h}=\left(\left(f_{\alpha}\right)_{\alpha \in \Lambda}, x, v, w\right) \in M
$$

be a $\sigma$-finite tripotent. It is clear that $\operatorname{spt}_{1} \boldsymbol{h}$ is countable. For each $\alpha \in \operatorname{spt}_{1} \boldsymbol{h}$ choose a complete tripotent $g_{\alpha} \in L^{\infty}\left(\mu_{\alpha}, C_{\alpha}\right)$ such that $g_{\alpha} \geq f_{\alpha}$, and for each $\alpha \in \Lambda \backslash \operatorname{spt}_{1} \boldsymbol{h}$ set $g_{\alpha}=0$.

Further, $x$ is a $\sigma$-finite tripotent in $N$, hence by Lemma $7.2(d)$ there is a $\sigma$-finite projection $y \in N$ with $x \in N_{2}(y)$.

Since $v$ is a $\sigma$-finite tripotent in $p V$, by Lemma 10.4 the set $\operatorname{spt}_{2} \boldsymbol{h}$ is countable. The final projection of $v$ satisfies $p_{f}(v) \leq \sum_{j \in \operatorname{spt}_{2} h} p_{j}$, so by Lemma 9.8( $c$ ) there is a projection $r \geq p_{i}(v)$ such that $r \sim \sum_{j \in \operatorname{spt}_{2} \boldsymbol{h}} p_{j}$, so we can choose a partial isometry $\tilde{v} \in V$ with $p_{i}(\tilde{v})=r$ and $p_{f}(\tilde{v})=\sum_{j \in \operatorname{spt}_{2} \boldsymbol{h}} p_{j}$.

Finally, $w$ is a $\sigma$-finite tripotent in $q W$, thus $p_{f}(w)$ is a $\sigma$-finite projection below $q$. It follows from Lemma 10.5 that there is a $\sigma$-finite properly infinite projection $s_{1}$ with $p_{f}(w) \leq s_{1} \leq q$. By Lemma 9.8(c) there is a projection $s_{2} \geq p_{i}(w)$ equivalent to $s_{1}$. Let $\tilde{w}$ be any partial isometry with $p_{i}(\tilde{w})=s_{2}$ and $p_{f}(\tilde{w})=s_{1}$.

Now it is clear that

$$
\boldsymbol{g}=\left(\left(g_{\alpha}\right)_{\alpha \in \Lambda}, y, \tilde{v}, \tilde{w}\right) \in \mathcal{T}(M)
$$

and $M_{2}(\boldsymbol{h}) \subseteq M_{2}(\boldsymbol{g})$. This completes the proof of assertion $(a)$.

We continue by proving $(b)$. Fix $\varphi, \psi \in M_{*} \backslash\{0\}$ such that

$$
\begin{aligned}
& s(\varphi)=\boldsymbol{h}=\left(\left(h_{\alpha}\right)_{\alpha \in \Lambda}, x(\boldsymbol{h}), v(\boldsymbol{h}), w(\boldsymbol{h})\right), \\
& s(\psi)=\boldsymbol{g}=\left(\left(g_{\alpha}\right)_{\alpha \in \Lambda}, x(\boldsymbol{g}), v(\boldsymbol{g}), w(\boldsymbol{g})\right) .
\end{aligned}
$$

It is clear that $\|\cdot\|_{\varphi}$ is weaker than $\|\cdot\|_{\psi}$ on $B_{M}$ if and only if 
- $\|\cdot\|_{\varphi}$ is weaker than $\|\cdot\|_{\psi}$ on $B_{L^{\infty}\left(\mu_{\alpha}, C_{\alpha}\right)}$ for each $\alpha \in \Lambda$,

- $\|\cdot\|_{\varphi}$ is weaker than $\|\cdot\|_{\psi}$ on $B_{N}$,

- $\|\cdot\|_{\varphi}$ is weaker than $\|\cdot\|_{\psi}$ on $B_{p V}$,

- $\|\cdot\|_{\varphi}$ is weaker than $\|\cdot\|_{\psi}$ on $B_{q W}$.

Observe that Proposition 9.7 yields that $\|\cdot\|_{\varphi}$ and $\|\cdot\|_{\psi}$ are equivalent on the closed unit ball of $L^{\infty}\left(\mu_{\alpha}, C_{\alpha}\right)$ whenever both $h_{\alpha}$ and $g_{\alpha}$ are nonzero. Further, clearly $\|\cdot\|_{\varphi}$ is weaker than $\|\cdot\|_{\psi}$ on $B_{N}$ if and only if $x(\boldsymbol{h}) \leq x(\boldsymbol{g})$ (by Proposition 7.5, but in fact this is an easy case).

Further, set

$$
u(\boldsymbol{g})=\sum_{j \in \mathrm{spt}_{2} \boldsymbol{g}} p_{j}, \quad u(\boldsymbol{h})=\sum_{j \in \mathrm{spt}_{2} \boldsymbol{h}} p_{j} .
$$

These are $\sigma$-finite projections in $V$ which belong to $p V$, thus there are $\tilde{\varphi}, \tilde{\psi} \in$ $(p V)_{*} \backslash\{0\}$ with $s(\tilde{\varphi})=u(\boldsymbol{h})$ and $s(\tilde{\psi})=u(\boldsymbol{g})$. By Lemma 9.9 and Proposition 7.5 we see that $\|\cdot\|_{\varphi}$ and $\|\cdot\|_{\tilde{\varphi}}$ are equivalent on $B_{p V}$ (and $\|\cdot\|_{\psi}$ and $\|\cdot\|_{\tilde{\psi}}$ as well). Now we deduce, via Proposition 7.5 , that $\|\cdot\|_{\varphi}$ is weaker than $\|\cdot\|_{\psi}$ on $B_{p V}$ if and only if $\operatorname{spt}_{2} \boldsymbol{h} \subseteq \mathrm{spt}_{2} \boldsymbol{g}$.

Now assertion $(b)$ follows easily.

Next we provide the following postponed proof.

Proof of Corollary 10.2. We use the notation from Theorem 10.1. By assertion (a) in the just quoted theorem, for each $n \in \mathbb{N}$, we can find an element $\boldsymbol{h}^{n} \in \mathcal{T}(M)$ such that $M_{2}\left(s\left(\varphi_{n}\right)\right) \subseteq M_{2}\left(\boldsymbol{h}^{n}\right)$ for each $n \in \mathbb{N}$. Fix the notation

$$
\boldsymbol{h}^{n}=\left(\left(f_{\alpha}^{n}\right)_{\alpha \in \Lambda}, x^{n}, v^{n}, w^{n}\right), \quad n \in \mathbb{N} .
$$

For any $\alpha \in \bigcup_{n} \operatorname{spt}_{1} h^{n}$ choose a complete tripotent $f_{\alpha} \in L^{\infty}\left(\mu_{\alpha}, C_{\alpha}\right)$ and set $f_{\alpha}=0$ for the remaining $\alpha \in \Lambda$. Further, set $x=\sup _{n} x^{n}$ and

$$
v=\sum_{j \in \cup_{n} \operatorname{spt}_{2} h^{n}} p_{j}
$$

Finally, $w^{n}$ is a partial isometry in $W$ such that its final projection $p_{f}\left(w^{n}\right)$ is a properly infinite $\sigma$-finite projection below $q$ for each $n \in \mathbb{N}$. By Lemma 10.5 there is a $\sigma$-finite properly infinite projection $r \in W$ with

$$
\sup _{n} p_{f}\left(w^{n}\right) \leq r \leq q .
$$

By Lemma 9.8 $(c)$ we can find, for each $n \in \mathbb{N}$, a projection $s_{n} \geq p_{i}\left(w^{n}\right)$ such that $s_{n} \sim r$. Then $s=\sup _{n} s_{n}$ is equivalent to $r$ by Lemma 9.8 $(a)$. So, we can fix a partial isometry $w \in W$ with initial projection $s$ and final projection $r$. Then

$$
\boldsymbol{h}=\left(\left(f_{\alpha}\right)_{\alpha \in \Lambda}, x, v, w\right) \in \mathcal{T}(M) .
$$

Choose $\varphi \in M_{*} \backslash\{0\}$ with $s(\varphi)=\boldsymbol{h}$. Then $\|\cdot\|_{\varphi_{n}}$ is weaker than $\|\cdot\|_{\varphi}$ on $B_{M}$ by Theorem 10.1(b) (and Proposition 7.5).

\section{Characterizations of Weakly compact sets and operators}

As a byproduct of our investigation we improve characterizations of weakly compact sets in preduals of $\mathrm{JBW}^{*}$-triples and of weakly compact operators on such spaces. We start by recalling the following known result. 
Theorem 11.1. 62, Theorem 1.1, Corollary 1.4 and Theorem 1.5] Let $K$ be a bounded subset in the predual of a $J B W^{*}$-triple $M$. Then the following are equivalent:

(a) $K$ is relatively weakly compact;

(b) There exist norm-one normal functionals $\varphi_{1}, \varphi_{2} \in M_{*}$ satisfying the following property: Given $\varepsilon>0$, there exists $\delta>0$ such that for every $x \in M$ with $\|x\| \leq 1$ and $\|x\|_{\varphi_{1}, \varphi_{2}}<\delta$, we have $|\phi(x)|<\varepsilon$ for every $\phi \in K$

(c) The restriction, $\left.K\right|_{C}$, of $K$ to each maximal abelian subtriple $C$ of $M$ is relatively weakly compact in $C_{*}$;

(d) For each tripotent $e \in M$ the restriction of $K$ to $M_{2}(e)$ is relatively weakly compact in $\left(M_{2}(e)\right)_{*}$;

(e) For any monotone decreasing sequence of tripotents $\left(e_{n}\right)$ in $M$ with $\left(e_{n}\right) \rightarrow 0$ in the weak* topology, we have $\lim _{n \rightarrow+\infty} \phi\left(e_{n}\right)=0$ uniformly for $\phi \in K$.

If $M$ is a $J B W^{*}$-algebra then statement (b) can be replaced with the following:

$\left(b^{\prime}\right)$ There exists a normal state $\psi \in M_{*}$ satisfying the following property: Given $\varepsilon>0$, there exists $\delta>0$ such that for every $x \in M$ with $\|x\| \leq 1$ and $\|x\|_{\psi}<\delta$, we have $|\phi(x)|<\varepsilon$ for each $\phi \in K$.

The equivalence $(a) \Leftrightarrow(b)$ is a generalization of Akemann's theorem [1] characterizing weakly compact sets in predual of von Neumann algebras. Recall that $\|x\|_{\varphi_{1}, \varphi_{2}}^{2}=\|x\|_{\varphi_{1}}^{2}+\|x\|_{\varphi_{2}}^{2}$, hence it gives also a more precise version of Proposition 7.9 on the relationship of strong* and Mackey topologies.

We also notice that a triple $C$ is abelian if the operators $L(a, b)$ and $L(x, y)$ commute for any choice $a, b, x, y \in C$ (cf. [16, p. 468]).

As observed in [17, pages 340-342] the previous theorem can be applied to characterize weakly compact operators from a complex Banach space into the predual of a $\mathrm{JBW}^{*}$-triple and from a $\mathrm{JB}^{*}$-triple into a complex Banach space. The concrete result in the latter case reads as follows.

Theorem 11.2. 65, Theorem 10] Let $E$ be a JB*-triple, $X$ a complex Banach space, and $T: E \rightarrow X$ a bounded linear operator. Then the following assertions are equivalent:

(i) $T$ is weakly compact;

(ii) There exist norm-one functionals $\varphi_{1}, \varphi_{2} \in E^{*}$ and a function $N:(0,+\infty) \rightarrow$ $(0,+\infty)$ such that

$$
\|T(x)\| \leq N(\varepsilon)\|x\|_{\varphi_{1}, \varphi_{2}}+\varepsilon\|x\|
$$

for all $x \in E$ and $\varepsilon>0$;

(iii) There exist a bounded linear operator $G$ from $E$ to a real (respectively, complex) Hilbert space and a function $N:(0,+\infty) \rightarrow(0,+\infty)$ such that

$$
\|T(x)\| \leq N(\varepsilon)\|G(x)\|+\varepsilon\|x\|
$$

for all $x \in E$ and $\varepsilon>0$.

This result is collected in the recent monograph [17] as Theorem 5.10.141. By quoting [17, it should be noted that "The above theorem is established in [20, Theorem 11], with $\|\cdot\|_{\varphi_{1}, \varphi_{2}}$ in condition (ii) replaced with $\|\cdot\|_{\varphi}$ for a single functional $\varphi$ in the unit sphere of $E^{*}$. Since this refinement depends on an affirmative answer to [17, Problem 5.10.131], it should remain in doubt." Problem 5.10.131 refers to 
the so-called Barton-Friedman conjecture for $\mathrm{JB}^{*}$-triples and the subtle difficulties appearing around the original statement of Grothendieck's inequality for JB*-triples published in 6] (see 60, 65, 61, [17, Subsection 5.10.4], 35] and the final remark in page 55 for more details). Summarizing, the problem whether in Theorem 11.2( $(i)$ (respectively, Theorem 11.1(b)) the seminorm of the form $\|\cdot\|_{\varphi_{1}, \varphi_{2}}$ can be replaced with a seminorm of the form $\|\cdot\|_{\varphi}$ for a single norm-one functional $\varphi \in E^{*}$ remains as an open question. Our next result provides a positive solution to these problems and proves the validity of the original statement in [20, Theorem 11].

Theorem 11.3. Let $K$ be a bounded subset in the predual of a $J B W^{*}$-triple $M$. Then $K$ is relatively weakly compact if and only if there exists a norm-one normal functional $\varphi \in M_{*}$ satisfying the following property: Given $\varepsilon>0$, there exists $\delta>0$ such that for every $x \in M$ with $\|x\| \leq 1$ and $\|x\|_{\varphi}<\delta$, we have $|\phi(x)|<\varepsilon$ for every $\phi \in K$.

Proof. The 'if part' follows from the implication $(b) \Rightarrow(a)$ in Theorem 11.1, whereas the 'only if part follows from the implication $(a) \Rightarrow(b)$ in Theorem 11.1 and Corollary 10.2 .

We can now provide a proof of the statement in [20, Theorem 11] and close a conjecture which has remained open for over eighteen years. The proof dissipates the commented doubts expressed in [17, page 341].

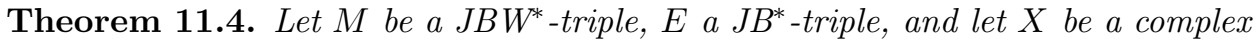
Banach space. Then the following statements hold:

(a) A bounded linear operator $T: X \rightarrow M_{*}$ is weakly compact if and only if there exists a norm-one functional $\varphi \in M_{*}$ and a function $N:(0,+\infty) \rightarrow(0,+\infty)$ such that

for all $a \in M$ and $\varepsilon>0$;

$$
\left\|T^{*}(a)\right\| \leq N(\varepsilon)\|a\|_{\varphi}+\varepsilon\|a\|
$$

(b) A bounded linear operator $T: E \rightarrow X$ is weakly compact if and only if there exists a norm-one functional $\varphi \in E^{*}$ and a function $N:(0,+\infty) \rightarrow(0,+\infty)$ such that

$$
\|T(a)\| \leq N(\varepsilon)\|a\|_{\varphi}+\varepsilon\|a\|
$$

for all $a \in E$ and $\varepsilon>0$.

Proof. In both statements the 'if parts' follow from Theorem 11.2 .

(a) Suppose $T: X \rightarrow M_{*}$ is weakly compact. Since $T\left(B_{X}\right) \subseteq M_{*}$ is relatively weakly compact, Theorem 11.3 implies the existence of a norm-one normal functional $\varphi \in M_{*}$ satisfying the following property: Given $\varepsilon>0$, there exists $\delta>0$ such that for every $a \in M$ with $\|a\| \leq 1$ and $\|a\|_{\varphi}<\delta$, we have $|T(x)(a)|<\varepsilon$ for every $x \in B_{X}$.

Given $a \in M \backslash\{0\}$, the element $b=\frac{a}{\|a\|+\delta^{-1}\|a\|_{\varphi}} \in B_{M}$ and satisfies $\|b\|_{\varphi}<\delta$, therefore $|T(x)(b)|<\varepsilon$ for every $x \in B_{X}$, equivalently,

$$
\left|T^{*}(a)(x)\right|=|T(x)(a)|<\varepsilon \delta^{-1}\|a\|_{\varphi}+\varepsilon\|a\|,
$$

for all $x \in B_{X}$, and thus

$$
\left\|T^{*}(a)\right\| \leq \varepsilon \delta^{-1}\|a\|_{\varphi}+\varepsilon\|a\| \text {, for all } a \in M .
$$

Statement $(b)$ follows from $(a)$ since, by virtue of Gantmacher's theorem, an operator $T: E \rightarrow X$ is weakly compact if and only if $T^{*}: X^{*} \rightarrow E^{*}$ is. 


\section{FINAL REMARKS AND OPEN PROBLEMS}

Theorem 4.1 says that the three measures of weak non-compactness considered in this paper coincide in preduals of $\mathrm{JBW}^{*}$-triples which complements the previous results from 46, 34. However, the mentioned results include explicit formulas for these measures. In fact, these formulas are substantially used in the proofs. In the present paper we do not get an explicit formula due to the procedure of the proof we use subsequence splitting property, Lemma 8.3 . Proposition 3.2 and Lemma 2.1 . So it is natural to ask whether there are some natural formulas for the De Blasi measure $\omega$. The first question deals with a special case of von Neumann algebras.

Question 12.1. Let $M$ be a semifinite von Neumann algebra with a fixed normal semifinite faithful trace $\tau$. Is there a formula for the De Blasi measure of weak noncompatness in $M_{*}$ in terms of the trace $\tau$ ? Is it so at least for finite $\sigma$-finite von Neumann algebras?

We note that the special case of commutative spaces $L^{1}(\mu)$ is settled in [46, Section 7].

Another possibility is to try to get quantitative versions of some characterizations of weakly compact sets in the predual of a $\mathrm{JBW}^{*}$-triple given in Theorem 11.1 (or in the improvement of its assertion (b) contained in Theorem 11.3). More precisely, we have the following question.

Question 12.2. Let $M$ be a JBW*-triple and let $A \subseteq M_{*}$ be a bounded set. Can $\omega(A)$ be expressed using quantitative versions of the characterizations from Theorem 11.1? In particular, is $\omega(A)$ equal (or at least equivalent) to the following quantities?

(b) $\inf _{\varphi \in S_{M_{*}}} \sup _{\phi \in A} \inf _{\delta>0} \sup \left\{|\phi(x)| ; x \in B_{M},\|x\|_{\varphi}<\delta\right\}$

(c) $\sup \left\{\omega\left(\left.A\right|_{C}\right) ; C \subseteq M\right.$ a maximal abelian subtriple $\}$;

(d) $\sup \left\{\omega\left(\left.A\right|_{M_{2}(e)}\right) ; e \in M\right.$ a tripotent $\}$;

(e) $\sup \left\{\begin{array}{cc}\limsup _{n \rightarrow \infty} \sup _{\phi \in A}\left|\phi\left(e_{n}\right)\right| ; & \left(e_{n}\right) \text { a decreasing sequence of tripotents } \\ \text { weak }^{*} \text {-converging to } 0\end{array}\right\}$.

Note that these quantities naturally correspond to the respective characterizations in Theorem 11.1 (or in the improvement of assertion (b) contained in Theorem 11.3). It is easy to check that all these quantities are bounded above by the De Blasi measure $\omega$, but the converse inequalities seem not to be obvious.

We investigated measures of weak non-compactness in preduals of $\mathrm{JBW}^{*}$-triples. Another possibility is to look at subsets of $\mathrm{JB}^{*}$-triples themselves. In this direction there is just one positive result - coincidence of measures of weak non-compactness for subsets of $c_{0}(\Gamma)$ (see [34, Theorem $\mathrm{C}$ ) ) and no negative result up to now. So, the following question seems to be natural.

Question 12.3. Are the above-considered measures of weak non-compactness equivalent (or even equal) for bounded subsets of a $J B W^{*}$-triple?

We have no idea how to approach this general question, so we formulate two important special cases.

Question 12.4. Let $K$ be a compact space. Are the above-considered measures of weak non-compactness equivalent (or even equal) for bounded subsets of $\mathcal{C}(K)$ ? Is it true for $K=[0,1]$ ? 
Question 12.5. Let $H$ be a Hilbert space. Are the above-considered measures of weak non-compactness equivalent (or even equal) for bounded subsets of $K(H)$, the space of compact operators on $H$ ?

Added during the revision process: Months after the submission of this paper we discovered a complete proof of the so-called Barton-Friedman conjecture for general $\mathrm{JB}^{*}$-triples, and a solution to Problem 5.10.131 in [17] (treated in page 53). The result is included in the recent preprint [35. This proof of the BartonFriedman conjecture offers an alternative approach to derive Theorems 11.3 and 11.4 as a straightforward consequences of [65, Theorem 10].

Acknowledgements: We would like to thank the anonymous referee for the time employed in writing a professional and thorough report with a wide list of constructive and enriching comments and suggestions.

\section{REFERENCES}

[1] Akemann, C. A. The dual space of an operator algebra. Trans. Amer. Math. Soc. 126 (1967), $286-302$.

[2] Aliprantis, C. D., And Border, K. C. Infinite-dimensional analysis, second ed. SpringerVerlag, Berlin, 1999. A hitchhiker's guide.

[3] Angosto, C., and Cascales, B. The quantitative difference between countable compactness and compactness. J. Math. Anal. Appl. 343, 1 (2008), 479-491.

[4] Angosto, C., And Cascales, B. Measures of weak noncompactness in Banach spaces. Topology Appl. 156, 7 (2009), 1412-1421.

[5] Astala, K., And Tylli, H.-O. Seminorms related to weak compactness and to Tauberian operators. Math. Proc. Cambridge Philos. Soc. 107, 2 (1990), 367-375.

[6] Barton, T., and Friedman, Y. Grothendieck's inequality for $J B^{*}$-triples and applications. J. London Math. Soc. (2) 36, 3 (1987), 513-523.

[7] Barton, T., and Friedman, Y. Bounded derivations of JB*-triples. Quart. J. Math. Oxford Ser. (2) 41, 163 (1990), 255-268.

[8] Barton, T., And Timoney, R. M. Weak*-continuity of Jordan triple products and its applications. Math. Scand. 59, 2 (1986), 177-191.

[9] Bohata, M., Hamhalter, J., and Kalenda, O. F. K. On Markushevich bases in preduals of von Neumann algebras. Israel J. Math. 214, 2 (2016), 867-884.

[10] Bohata, M., Hamhalter, J., and Kalenda, O. F. K. Decompositions of preduals of JBWand JBW*-algebras. J. Math. Anal. Appl. 446, 1 (2017), 18-37.

[11] Bohata, M., Hamhalter, J., Kalenda, O. F. K., Peralta, A. M., and Pfitzner, H. Preduals of JBW*-triples are 1-Plichko spaces. Q. J. Math. 69, 2 (2018), 655-680.

[12] Bourgain, J., and Rosenthal, H. P. Martingales valued in certain subspaces of $L^{1}$. Israel J. Math. 37, 1-2 (1980), 54-75.

[13] Braun, R., Kaup, W., And Upmeier, H. A holomorphic characterization of Jordan $C^{*}$ algebras. Math. Z. 161, 3 (1978), 277-290.

[14] Bunce, L. J. Norm preserving extensions in JBW*-triple preduals. Q. J. Math. 52, 2 (2001), 133-136.

[15] Bunce, L. J., Fernández-Polo, F. J., Martínez Moreno, J., and Peralta, A. M. A Saitô-Tomita-Lusin theorem for JB*-triples and applications. Q. J. Math. 57, 1 (2006), 3748.

[16] Cabrera García, M., and Rodríguez Palacios, A. Non-associative normed algebras. Vol. 1, vol. 154 of Encyclopedia of Mathematics and its Applications. Cambridge University Press, Cambridge, 2014. The Vidav-Palmer and Gelfand-Naimark theorems.

[17] Cabrera García, M., and Rodríguez Palacios, A. Non-associative normed algebras. Vol. 2, vol. 167 of Encyclopedia of Mathematics and its Applications. Cambridge University Press, Cambridge, 2018. Representation theory and the Zel'manov approach.

[18] Cascales, B., Kalenda, O. F. K., and Spurný, J. A quantitative version of James's compactness theorem. Proc. Edinb. Math. Soc. (2) 55, 2 (2012), 369-386. 
[19] CHU, C.-H. Jordan structures in geometry and analysis, vol. 190 of Cambridge Tracts in Mathematics. Cambridge University Press, Cambridge, 2012.

[20] Chu, C.-H., And Iochum, B. Weakly compact operators on Jordan triples. Math. Ann. 281, 3 (1988), 451-458.

[21] De Blasi, F. S. On a property of the unit sphere in a Banach space. Bull. Math. Soc. Sci. Math. R. S. Roumanie (N.S.) 21(69), 3-4 (1977), 259-262.

[22] Diestel, J. Sequences and series in Banach spaces, vol. 92 of Graduate Texts in Mathematics. Springer-Verlag, New York, 1984.

[23] Edwards, C. M., and Rüttimann, G. T. On the facial structure of the unit balls in a JBW*-triple and its predual. J. London Math. Soc. (2) 38, 2 (1988), 317-332.

[24] Edwards, C. M., and Rüttimann, G. T. Exposed faces of the unit ball in a JBW*-triple. Math. Scand. 82, 2 (1998), 287-304.

[25] Fabian, M., Habala, P., Hájek, P., Montesinos Santalucía, V., Pelant, J., and ZiZLER, V. Functional analysis and infinite-dimensional geometry. CMS Books in Mathematics/Ouvrages de Mathématiques de la SMC, 8. Springer-Verlag, New York, 2001.

[26] Fabian, M., Hájek, P., Montesinos, V., And Zizler, V. A quantitative version of Krein's theorem. Rev. Mat. Iberoamericana 21, 1 (2005), 237-248.

[27] Fernández-Polo, F. J., Peralta, A. M., and Ramírez, M. I. A Kadec-Pelczyński dichotomy-type theorem for preduals of JBW*-algebras. Israel J. Math. 208, 1 (2015), 45-78.

[28] Friedman, Y., And Russo, B. Solution of the contractive projection problem. J. Funct. Anal. 60, 1 (1985), 56-79.

[29] Friedman, Y., And Russo, B. Structure of the predual of a $J B W^{*}$-triple. J. Reine Angew. Math. 356 (1985), 67-89.

[30] Friedman, Y., And Russo, B. The Gel'fand-Naĭmark theorem for JB*-triples. Duke Math. J. 53, 1 (1986), 139-148.

[31] Granero, A. S. An extension of the Krein-Šmulian theorem. Rev. Mat. Iberoam. 22, 1 (2006), 93-110.

[32] Granero, A. S., Hernández, J. M., and Pfitzner, H. The $\operatorname{distance} \operatorname{dist}(\mathcal{B}, X)$ when $\mathcal{B}$ is a boundary of $B\left(X^{* *}\right)$. Proc. Amer. Math. Soc. 139, 3 (2011), 1095-1098.

[33] Grothendieck, A. Espaces vectoriels topologiques. Instituto de Matemática Pura e Aplicada, Universidade de São Paulo, São Paulo, 1954.

[34] Hamhalter, J., ANd Kalenda, O. F. K. Measures of weak non-compactness in spaces of nuclear operators. Math. Z. 292, 1-2 (2019), 453-471.

[35] Hamhalter, J., Kalenda, O. F. K., Peralta, A. M., and Pfitzner, H. Grothendieck's inequalities for JB-triples: Proof of the Barton-Friedman conjecture. arXiv:1903.08931.

[36] Hanche-Olsen, H., ANd Størmer, E. Jordan operator algebras, vol. 21. Pitman Advanced Publishing Program, 1984.

[37] Harmand, P., Werner, D., And Werner, W. M-ideals in Banach spaces and Banach algebras, vol. 1547 of Lecture Notes in Mathematics. Springer-Verlag, Berlin, 1993.

[38] Ho, T., Martinez-Moreno, J., Peralta, A. M., and Russo, B. Derivations on real and complex JB *-triples. J. London Math. Soc. (2) 65, 1 (2002), 85-102.

[39] Horn, G. Klassifikation der JBW*-Tripel vomm Type 1. Dissertation. University of Tübingen, 1984.

[40] Horn, G. Characterization of the predual and ideal structure of a JBW*-triple. Math. Scand. 61, 1 (1987), 117-133.

[41] Horn, G. Classification of JBW*-triples of type I. Math. Z. 196, 2 (1987), 271-291.

[42] Horn, G., AND Neher, E. Classification of continuous $J B W^{*}$-triples. Trans. Amer. Math. Soc. 306, 2 (1988), 553-578.

[43] Iochum, B. Cônes autopolaires et algèbres de Jordan, vol. 1049 of Lecture Notes in Mathematics. Springer-Verlag, Berlin, 1984.

[44] Isidro, J. M., Kaup, W., And Rodríguez-Palacios, A. On real forms of JB*-triples. Manuscripta Math. 86, 3 (1995), 311-335.

[45] Jarchow, H. On weakly compact operators on $C^{*}$-algebras. Math. Ann. 273, 2 (1986), 341343.

[46] KaČena, M., Kalenda, O. F. K., and Spurný, J. Quantitative Dunford-Pettis property. Adv. Math. 234 (2013), 488-527.

[47] Kadec, M. I., ANd PęczyŃski, A. Bases, lacunary sequences and complemented subspaces in the spaces $L_{p}$. Studia Math. 21 (1961/1962), 161-176. 
[48] Kadison, R. V. Isometries of operator algebras. Ann. Of Math. (2) 54 (1951), 325-338.

[49] Kadison, R. V., And Ringrose, J. R. Fundamentals of the theory of operator algebras. Vol. I, vol. 100 of Pure and Applied Mathematics. Academic Press, Inc. [Harcourt Brace Jovanovich, Publishers], New York, 1983. Elementary theory.

[50] Kadison, R. V., And Ringrose, J. R. Fundamentals of the theory of operator algebras. Vol. II, vol. 16 of Graduate Studies in Mathematics. American Mathematical Society, Providence, RI, 1997. Advanced theory, Corrected reprint of the 1986 original.

[51] Kalenda, O., Pfitzner, H., and SpurnÝ, J. On quantification of weak sequential completeness. J. Funct. Anal. 260, 10 (2011), 2986-2996.

[52] KAUP, W. A Riemann mapping theorem for bounded symmetric domains in complex Banach spaces. Math. Z. 183, 4 (1983), 503-529.

[53] KAUP, W. Contractive projections on Jordan $C^{*}$-algebras and generalizations. Math. Scand. 54, 1 (1984), 95-100.

[54] Kaup, W. On real Cartan factors. Manuscripta Math. 92, 2 (1997), 191-222.

[55] Kaup, W., And Upmeier, H. Jordan algebras and symmetric Siegel domains in Banach spaces. Math. Z. 157, 2 (1977), 179-200.

[56] Knaust, H., And Odell, E. On $c_{0}$ sequences in Banach spaces. Israel J. Math. 67, 2 (1989), 153-169.

[57] Loos, O. Bounded symmetric domains and Jordan pairs. Lecture Notes, Univ. California at Irvine, 1977.

[58] NeHER, E. Jordan triple systems by the grid approach, vol. 1280 of Lecture Notes in Mathematics. Springer-Verlag, Berlin, 1987.

[59] Pedersen, G. K. $C^{*}$-algebras and their automorphism groups, vol. 14 of London Mathematical Society Monographs. Academic Press, Inc. [Harcourt Brace Jovanovich, Publishers], London-New York, 1979.

[60] Peralta, A. M. Little Grothendieck's theorem for real JB*-triples. Math. Z. 237, 3 (2001), 531-545.

[61] Peralta, A. M. New advances on the Grothendieck's inequality problem for bilinear forms on JB*-triples. Math. Inequal. Appl. 8, 1 (2005), 7-21.

[62] Peralta, A. M. Some remarks on weak compactness in the dual space of a JB*-triple. Tohoku Math. J. (2) 58, 2 (2006), 149-159.

[63] Peralta, A. M. Positive definite hermitian mappings associated with tripotent elements. Expo. Math. 33, 2 (2015), 252-258.

[64] Peralta, A. M., And Pfitzner, H. The Kadec-Pełczyński-Rosenthal subsequence splitting lemma for JBW*-triple preduals. Studia Math. 227, 1 (2015), 77-95.

[65] Peralta, A. M., and Rodríguez Palacios, A. Grothendieck's inequalities for real and complex JBW*-triples. Proc. London Math. Soc. (3) 83, 3 (2001), 605-625.

[66] Randrianantoanina, N. Kadec-Pełczyński decomposition for Haagerup $L^{p}$-spaces. Math. Proc. Cambridge Philos. Soc. 132, 1 (2002), 137-154.

[67] Raynaud, Y., and Xu, Q. On subspaces of non-commutative $L_{p}$-spaces. J. Funct. Anal. 203, 1 (2003), 149-196.

[68] RodríGuez-Palacios, A. On the strong* topology of a JBW*-triple. Quart. J. Math. Oxford Ser. (2) 42, 165 (1991), 99-103.

[69] Rodríguez-Palacios, A. Jordan structures in analysis. In Jordan algebras (Oberwolfach, 1992). de Gruyter, Berlin, 1994, pp. 97-186.

[70] Rodríguez Palacios, A., and Cabrera García, M. A new proof of the Barton-Timoney theorem on the bidual of a $J B^{*}$-triple. Math. Nachr. 292, 3 (2019), 640-644.

[71] RYAN, R. A. Introduction to tensor products of Banach spaces. Springer Science \& Business Media, 2013.

[72] SaKai, S. $C^{*}$-algebras and $W^{*}$-algebras. Springer Science \& Business Media, 2012.

[73] Schlüchtermann, G., And Wheeler, R. F. On strongly WCG Banach spaces. Math. Z. 199, 3 (1988), 387-398.

[74] Sherman, D. On the dimension theory of von Neumann algebras. Math. Scand. 101, 1 (2007), 123-147.

[75] Stacho, L. L. A projection principle concerning biholomorphic automorphisms. Acta Sci. Math 44 (1982), 99-124. 
[76] Strătilă, Ş., AND Zsidó, L. Lectures on von Neumann algebras. Editura Academiei, Bucharest; Abacus Press, Tunbridge Wells, 1979. Revision of the 1975 original, Translated from the Romanian by Silviu Teleman.

[77] Takesaki, M. Theory of operator algebras. I. Springer-Verlag, New York-Heidelberg, 1979.

[78] Wright, J. D. M. Jordan $C^{*}$-algebras. Michigan Math. J. 24, 3 (1977), 291-302.

[79] Youngson, M. A. A Vidav theorem for Banach Jordan algebras. Math. Proc. Cambridge Philos. Soc. 84, 2 (1978), 263-272.

Czech Technical University in Prague, Faculty of Electrical Engineering, Department of Mathematics, Technicka 2, 166 27, Prague 6, Czech Republic

E-mail address: hamhalte@math.feld.cvut.cz

Charles University, Faculty of Mathematics and Physics, Department of Mathematical Analysis, Sokolovská 86, 18675 Praha 8, Czech Republic

E-mail address: kalenda@karlin.mff.cuni.cz

Departamento de Análisis Matemático, Facultad de Ciencias, Universidad de GranaDA, 18071 Granada, Spain.

E-mail address: aperalta@ugr.es

Institut Denis Poisson, Université d’Orléans, Université de Tours, CNRS, Rue de Chartres, BP 6759, F-45067 Orléans Cedex 2, France

E-mail address: hermann.pfitzner@univ-orleans.fr 